\title{
INFINITE DIMENSIONAL LINEAR SYSTEMS WITH UNBOUNDED CONTROL AND OBSERVATION: A FUNCTIONAL ANALYTIC APPROACH
}

\author{
DIETMAR SALAMON
}

\begin{abstract}
The object of this paper is to develop a unifying framework for the functional analytic representation of infinite dimensional linear systems with unbounded input and output operators. On the basis of the general approach new results are derived on the wellposedness of feedback systems and on the linear quadratic control problem. The implications of the theory for large classes of functional and partial differential equations are discussed in detail.
\end{abstract}

1. Introduction. For large classes of infinite dimensional control systems an adequate mathematical representation leads to unbounded input and output operators. In partial differential equations this is the case if the control acts through the boundary and if the measurements can only be taken at a few points of the spatial domain. Analogous phenomena occur in functional differential equations if there are delays in the input and output variables.

This paper presents a unifying abstract framework for the study of infinite dimensional linear systems which allows for unbounded control and observation. The main emphasis has been to keep the theory in a simple and elegant form and still to cover most of the known examples of wellposed, linear, time invariant infinite dimensional control systems. The general approach is then used to derive new results on the wellposedness of feedback systems and on the linear quadratic control problem. Furthermore, it is shown how large classes of functional and partial differential equations can be represented within the abstract functional analytic framework.

The relevance of unbounded input and output operators both from a theoretical and from a practical point of view has been recognized for a long time in the literature on the mathematical theory of infinite dimensional control systems. Without attempting to give a complete overview we mention the classical work by Lions [28], Lions and Magenes [29] as well as the early papers by Fattorini [12], Lukes and Russell [30], Russell [36, 37, 38] and the more recent book by Curtain and

Received by the editors January 23, 1985 and, in revised form, January 2, 1986.

1980 Mathematics Subject Classification (1985 Revision). Primary 93C25, 34G10, 49A27, 34K05, $35 \mathrm{~K} 35,35 \mathrm{~L} 35$.

Key words and phrases. Representation of infinite dimensional systems, semigroups, boundary control, feedback, linear quadratic control.

This material is based upon work supported by the National Science Foundation under Grant No. DMS-8210950, Mod. 1. 
Pritchard [6]. In recent years more attention has been paid to the abstract representation of boundary control systems. In the context of partial differential equations we refer to Balakrishnan [2], Washburn [45], Ho and Russell [19], Lasiecka and Triggiani [24], and in the context of functional differential equations to Ichikawa [20], Delfour [10], Salamon [40], Delfour and Karrakchou [11], Pritchard and Salamon [34].

Despite these efforts there are certain classes of wellposed infinite dimensional systems for which a satisfactory functional analytic representation has not yet been developed. Among these there are the examples discussed in $\$ \S 6$ and 7.3. The main feature of these systems is that the input and output operators are in a sense more unbounded than the operator which describes the dynamics of the free system. In particular, the wave equation in $\$ 7.3$ has been one of the main motivating examples for the development of our general approach.

This general approach is discussed in detail in $\$ 2$. The important new feature of the abstract semigroup control system in $\$ 2.1$ is the representation of the output and the introduction of the operator $T_{\mu}$. The development of this new structure has turned out to be necessary in order to allow for enough unboundedness in the input and output operators. If either the input or the output operator is strictly unbounded, an equivalent representation of the infinite dimensional system is derived in $\S \S 2.2$ and 2.3 leading to the concepts of an "abstract boundary control system" and an "abstract point observation process". These two concepts are dual to each other while the concept of an "abstract semigroup control system" is self dual ( $\$ 3)$. Based on the fundamental theory of $\$ 2$ a new perturbation result is derived in $\$ 4$. $\$ 5$ deals with the linear quadratic control problem for the class of systems discussed in $\$ 2$ without further restrictions. In particular, the optimal control is characterized in terms of the dual system and conditions are given under which the optimal control is differentiable. Furthermore, it is shown that the optimal control satisfies an unbounded feedback law and is related to a Riccati type equation. A very general class of functional differential equations is discussed in $\$ 6$. In $\$ 7$ it is shown how both parabolic and hyperbolic partial differential equations can be described within the framework of $\S 2$.

\section{Three basic concepts.}

2.1. Semigroup control systems. An abstract semigroup control system (SCS) is described by the equation

$$
\dot{x}(t)=A x(t)+B u(t), \quad t \geqslant 0, x(0)=x_{0},
$$

where $u(t) \in U$ is the input, $W \subset H \subset V$ are Hilbert spaces with continuous, dense injections and $A \in \mathscr{L}(W, H) \cap \mathscr{L}(H, V), B \in \mathscr{L}(U, V)$ where $U$ is also a Hilbert space.

Remark 2.1. If $A: \mathscr{D}(A) \rightarrow H$ is a closed, densely defined operator on a Hilbert space $H$, then $W=\mathscr{D}(A)$ and $V^{*}=\mathscr{D}\left(A^{*}\right)$ can be made into Hilbert spaces with the respective graph norms. Identifying $H$ with its dual we obtain $W \subset H \subset V$ and $V^{*} \subset H \subset W^{*}$ with continuous, dense injections. Furthermore, $A$ and $A^{*}$ can now be regarded as bounded operators from $W$, or respectively $V^{*}$, into $H$. By duality, 
we obtain the extensions $A \in \mathscr{L}(H, V), A^{*} \in \mathscr{L}\left(H, W^{*}\right)$. If $A$ has a nonempty resolvent set, then $W=\{x \in H \mid A x \in H\}$ and the bounded extension $A \in$ $\mathscr{L}(H, V)$ coincides with the adjoint of the unbounded restriction $A^{*}: \mathscr{D}\left(\left(A^{*}\right)^{2}\right) \rightarrow$ $V^{*}$. The same holds for $A^{*}$. Finally, we point out that whenever $W \subset H$ with a continuous, dense injection and $A \in \mathscr{L}(W, H)$ has a nonempty resolvent set, then the norm on $W$ is equivalent to the graph norm of $A$.

Now we will discuss the output of the SCS (2.1.1) in a Hilbert space $Y$. The output of the free system $(u(t) \equiv 0)$ can be described by an operator $C \in \mathscr{L}(W, Y)$ if $x(t) \in W$ for every $t \geqslant 0$. In order to describe the output of the forced motions of $(2.1 ; 1)$ let us assume that $\mu I-A: W \rightarrow H$ is boundedly invertible for some $\mu \in R$. Then every solution $x(\cdot) \in \mathscr{C}^{1}[0, T ; H]$ of $(2.1 ; 1)$ can be written in the form

$$
x(t)=(\mu I-A)^{-1}(\mu x(t)-\dot{x}(t))+(\mu I-A)^{-1} B u(t) .
$$

Hence $x(t) \notin W$ unless $B u(t) \in H$. Therefore the operator $C$ alone is not enough to describe the output of the forced motions. Another operator $T_{\mu} \in \mathscr{L}(U, Y)$ is needed. Then as motivated by $(2.2)$ we can define the output of $(2.1 ; 1)$ by

$$
y(t)=C(\mu I-A)^{-1}(\mu x(t)-\dot{x}(t))+T_{\mu} u(t)
$$

whenever $x(\cdot) \in \mathscr{C}^{1}[0, T ; H]$ satisfies $(2.1 ; 1)$. In order to make sure that the expression $(2.1 ; 2)$ is independent of $\mu$, we have to assume that the operator family $T_{\mu} \in \mathscr{L}(U, Y), \mu \notin \sigma(A)$, satisfies a certain compatibility condition. The following hypothesis summarizes all the assumptions imposed on $A, B, C$ and $T_{\mu}$.

$$
\text { The operator } \mu I-A: W \rightarrow H \text { is boundedly invertible for }
$$
some $\mu \in R, V^{*}=\mathscr{D}\left(A^{*}\right)$,

$$
T_{\mu}-T_{\lambda}=(\lambda-\mu) C(\mu I-A)^{-1}(\lambda I-A)^{-1} B
$$

for all $\lambda, \mu \notin \sigma(A)$.

At some places we need in addition that the input and output operators are strictly unbounded (with respect to $H$ ), that is,

$$
\begin{gathered}
\text { range } B \cap H=\{0\}, \\
\text { range } C^{*} \cap H=\{0\} .
\end{gathered}
$$

REMARKS 2.2. (i) The compatibility condition (2.3) guarantees that the expression $(2.1 ; 2)$ for the output of the system is independent of $\mu$.

(ii) The operator family $T_{\mu} \in \mathscr{L}(U, Y)$ is analytic on $\mathbf{C} \backslash \sigma(A)$ and has to be understood as the transfer operator which determines the input/output relationship of (2.1) in the frequency domain. It generalizes the expression $C(\mu I-A)^{-1} B$ which does not make sense if both $B$ and $C$ are strictly unbounded with respect to $H$.

(iii) If either range $B \subset H$ or $C$ extends to a bounded operator from $H$ into $Y$, then $T_{\mu}=C(\mu I-A)^{-1} B+D$ for some $D \in \mathscr{L}(U, Y)$. Therefore $T_{\mu}$ includes the possibility of a direct input/output relation. 
The next hypothesis is related to the homogeneous equation $(2.1 ; 1)(u(t) \equiv 0)$.

The operator $A: \mathscr{D}(A)=W \rightarrow H$ is the infinitesimal generator of a strongly continuous semigroup $S(t) \in \mathscr{L}(H)$.

If (S0) and (S1) are satisfied, then $S(t)$ is also a strongly continuous semigroup on $W$ and $V$ and the infinitesimal generator of $S(t) \in \mathscr{L}(V)$ is given by the extended operator $A: H \rightarrow V$ (Remark 2.1). The next Lemma is a well-known result in semigroup theory and summarizes the consequences of hypothesis (S1) for the inhomogeneous equation $(2.1 ; 1)$.

LemmA 2.3. Let (S0) and (S1) be satisfied, let $x_{0} \in H$ and $u(\cdot) \in W^{1,2}[0, T ; U]$ be given and define

$$
x(t)=S(t) x_{0}+\int_{0}^{t} S(t-s) B u(s) d s, \quad 0 \leqslant t \leqslant T .
$$

Then $x(\cdot) \in \mathscr{C}[0, T ; H] \cap \mathscr{C}^{1}[0, T ; V]$ and

$$
\dot{x}(t)=A x(t)+B u(t)=S(t)\left[A x_{0}+B u(0)\right]+\int_{0}^{t} S(t-s) B \dot{u}(s) d s
$$

for $0 \leqslant t \leqslant T$. If moreover $u(\cdot) \in W^{2,2}[0, T ; U]$ and $A x_{0}+B u(0) \in H$, then $x(\cdot) \in$ $\mathscr{C}^{1}[0, T ; H]$.

Let (S0) and (S1) be satisfied and let $u(\cdot) \in W^{2,2}[0, T ; U]$ and $x_{0} \in H$ satisfy $A x_{0}+B u(0) \in H$. Then we denote by $x(t)=x\left(t ; x_{0}, u\right)$ the corresponding unique solution of $(2.1 ; 1)$ which is given by $(2.6)$ and by $y(t)=y\left(t ; x_{0}, u\right)$ the associated output $(2.1 ; 2)$. The next hypotheses are related to the input/state, the state/output and the input/output relationship of the SCS (2.1).

(S1) is satisfied and there exists a $c>0$ such that the following inequality holds for all $u(\cdot) \in W^{1,2}[0, T ; U]$ :

$$
\left\|\int_{0}^{T} S(T-s) B u(s) d s\right\|_{H} \leqslant c\|u(\cdot)\|_{L^{2}[0, T: U]} .
$$

(S1) is satisfied and there exists a $c>0$ such that the following inequality holds for all $x \in W$ :

$$
\|C S(\cdot) x\|_{L^{2}[0, T: Y]} \leqslant c\|x\|_{H} .
$$

(S1) is satisfied and there exists a $c>0$ such that the following inequality holds for every $u(\cdot) \in W^{2,2}[0, T ; U]$ with $u(0)=0$ :

$$
\|y(\cdot ; 0, u)\|_{L^{2}[0, T ; Y]} \leqslant c\|u(\cdot)\|_{L^{2}[0, T ; U]} .
$$

DEFINITION 2.4. The SCS (2.1) is said to be wellposed if (S0)-(S4) are satisfied. If the SCS (2.1) is wellposed and $x_{0} \in H, u(\cdot) \in L^{2}[0, T ; U]$ are given, we define $x(t)=x\left(t ; x_{0}, u\right)$ by $(2.6)$ and $y(t)=y\left(t ; x_{0}, u\right)$ by continuous extension of the expression $(2.1 ; 2)$ using $(\mathrm{S} 3)$ and $(\mathrm{S} 4) . y(t)$ is said to be the weak output of the SCS (2.1). 
By definition, the weak output $y\left(t ; x_{0}, u\right)$ of the wellposed SCS (2.1) has to satisfy $(2.1 ; 2)$ only if $u(\cdot) \in W^{2,2}[0, T ; H], u(0)=0, x_{0} \in W$. It is not immediately obvious that $y(t)$ also satisfies $(2.1 ; 2)$ in general whenever $x\left(\cdot ; x_{0}, u\right) \in$ $W^{1,2}[0, T ; H]$. We establish this in the next lemma along with some differentiability properties of the solutions and outputs of (2.1).

LEMmA 2.5. Suppose that the SCS (2.1) is wellposed, let $x_{0} \in H, u(\cdot) \in L^{2}[0, T ; U]$ be given and let $x(t)=x\left(t ; x_{0}, u\right), y(t)=y\left(t ; x_{0}, u\right)$ be defined as above. Then the following statements hold.

(i) $x\left(\cdot ; x_{0}, u\right) \in \mathscr{C}[0, T ; H] \cap W^{1.2}[0, T ; V]$ satisfies $(2.1 ; 1)$ for almost every $t \in$ $[0, T]$.

(ii) If $x\left(\cdot ; x_{0}, u\right) \in W^{1,2}[0, T ; H]$ then $(2.1 ; 2)$ holds for almost every $t \in[0, T]$.

(iii) If $u(\cdot) \in W^{1,2}[0, T ; U]$ and $A x_{0}+B u(0) \in H$, then $x\left(\cdot ; x_{0}, u\right) \in \mathscr{C}^{1}[0, T ; H]$, $y\left(\cdot ; x_{0}, u\right) \in W^{1,2}[0, T ; Y]$ satisfy $\dot{x}\left(t ; x_{0}, u\right)=x\left(t ; A x_{0}+B u(0), \dot{u}\right)$ and $\dot{y}\left(t ; x_{0}, u\right)$ $=y\left(t ; A x_{0}+B u(0), \dot{u}\right)$ for (almost) every $t \in[0, T]$.

Proof. The continuity of $x(t)$ in $H$ follows from standard estimates using (S2) (see [40, Theorem 1.3.4]). Moreover, $(2.1 ; 1)$ follows from the fact that the equation

$$
x\left(t ; x_{0}, u\right)=x_{0}+\int_{0}^{t}\left[A x\left(s ; x_{0}, u\right)+B u(s)\right] d s
$$

holds for $0 \leqslant t \leqslant T, u(\cdot) \in W^{1,2}[0, T ; U], x_{0} \in H$ (Lemma 2.3) and that both sides of this equation depend continuously on $x_{0} \in H$ and $u(\cdot) \in L^{2}[0, T ; U]$. This proves statement (i).

In order to establish statement (ii) we have to make use of the calculations leading to equation (3.4) in the proof of Theorem 3.3 below. Let us fix $v(\cdot) \in W^{2,2}[0, T ; Y]$, $v(T)=0$, let $z(s)=z(s ; 0, v)$ be given by (3.3) and define

$$
w(s)=B^{*}\left(\mu I-A^{*}\right)^{-1}(\mu z(s)+\dot{z}(s))+T_{\mu}^{*} v(s) \text { for } 0 \leqslant s \leqslant T .
$$

Then it follows from (3.4) that the equation

$$
\int_{0}^{T}\left\langle v(t), y\left(t ; x_{0}, u\right)\right\rangle_{y} d t=\left\langle z(0), x_{0}\right\rangle_{H}+\int_{0}^{T}\langle w(s), u(s)\rangle_{U} d s
$$

holds for $x_{0} \in W, u(\cdot) \in W^{2,2}[0, T ; U], u(0)=0$, and hence, by continuous dependence for all $x_{0} \in H, u(\cdot) \in L^{2}[0, T ; U]$. If moreover $x\left(\cdot ; x_{0}, u\right) \in W^{1,2}[0, T ; H]$, we may define $\tilde{y}(t)$ by $(2.1 ; 2)$ and use $(3.4)$ once again to obtain

$$
\int_{0}^{T}\left\langle v(t), y\left(t ; x_{0}, u\right)-\tilde{y}(t)\right\rangle_{Y} d t=0
$$

Since the set of all $v(\cdot) \in W^{2,2}[0, T ; Y]$ with $v(T)=0$ is dense in $L^{2}[0, T ; Y]$ we conclude that $y=\tilde{y}$.

In order to establish statement (iii) let us choose $u(\cdot) \in W^{3.2}[0, T ; U]$ with $A x_{0}+B u(0) \in H$ and $A\left[A x_{0}+B u(0)\right]+B \dot{u}(0) \in H$. Then it follows from Lemma 2.3 that

$$
x\left(t ; x_{0}, u\right)=x_{0}+\int_{0}^{t} x\left(s ; A x_{0}+B u(0), \dot{u}\right) d s, \quad 0 \leqslant t \leqslant T
$$


and that $x\left(\cdot ; A x_{0}+B u(0), \dot{u}\right) \in \mathscr{C}^{1}[0, T ; H]$. Hence we can apply statement (ii) to both $y\left(t ; x_{0}, u\right)$ and $y\left(t ; A x_{0}+B u(0), \dot{u}\right)$ and obtain with the help of the above equation that

$$
y\left(t ; x_{0}, u\right)=y\left(0 ; x_{0}, u\right)+\int_{0}^{t} y\left(s ; A x_{0}+B u(0), \dot{u}\right) d s, \quad 0 \leqslant t \leqslant T .
$$

Hence statement (iii) follows from the fact that both sides in these two equations depend continuously on $x_{0} \in H, A x_{0}+B u(0) \in H$ and $u \in W^{1,2}[0, T ; U]$. Here we need the fact that $F=\{(x, u, z) \in H \times U \times H \mid A x+B u=z\}$ contains $\{(x, u, z)$ $\in F \mid A z \in H+$ range $B\}$ as a dense subspace. In fact, given $(x, u, z) \in F$ choose $w_{k} \in W$ converging to $\mu x-z \in H$ and define $x_{k}=(\mu I-A)^{-1}\left(w_{k}+B u\right), z_{k}=$ $A x_{k}+B u$. Then $\left(x_{k}, u, z_{k}\right)$ converges to $(x, u, z)$ in $F$ and $A z_{k}+\mu B u \in H$.

If the SCS (2.1) is wellposed then we introduce for notational convenience the operators $\mathscr{B}(T) \in \mathscr{L}\left(L^{2}[0, T ; U] ; H\right), \quad \mathscr{C}(T) \in \mathscr{L}\left(H, L^{2}[0, T ; Y]\right), \quad \mathscr{T}(T) \in$ $\mathscr{L}\left(L^{2}[0, T ; U], L^{2}[0, T ; Y]\right)$ such that

$$
\begin{gathered}
x\left(T ; x_{0}, u\right)=S(T) x_{0}+\mathscr{B}(T) u \in H, \\
y\left(\cdot ; x_{0}, u\right)=\mathscr{C}(T) x_{0}+\mathscr{T}(T) u \in L^{2}[0, T ; Y],
\end{gathered}
$$

for $x_{0} \in H$ and $u(\cdot) \in L^{2}[0, T ; U]$. For $t \leqslant T$ we also introduce the left shift operator $\sigma_{t}$ and the restriction operator $\rho_{t}$ from $L^{2}[0, T ; U]$ into $L^{2}[0, t ; U]$ by defining

$$
\left(\sigma_{t} u\right)(s)=u(s+T-t), \quad\left(\rho_{t} u\right)(s)=u(s), \quad 0 \leqslant s \leqslant t,
$$

for $u(\cdot) \in L^{2}[0, T ; U]$. Then $\sigma_{t}^{*}: L^{2}[0, t ; U] \rightarrow L^{2}[0, T ; U]$ is the right shift operator and $\rho_{t}^{*}$ is the extension operator. They are given by

$$
\begin{aligned}
\left(\sigma_{t}^{*} u\right)(s) & = \begin{cases}0, & 0<s<T-t, \\
u(s+t-T), & T-t<s<T,\end{cases} \\
\left(\rho_{t}^{*} u\right)(s) & = \begin{cases}u(s), & 0<s<t, \\
0, & t<s<T,\end{cases}
\end{aligned}
$$

for $u(\cdot) \in L^{2}[0, t ; U]$. The analogous operators on $L^{2}[0, T ; Y]$ will also be denoted by $\sigma_{t}, \rho_{t}, \sigma_{t}^{*}, \rho_{t}^{*}$. The following relations between the various operators express the linearity and time invariance of the SCS (2.1). They can be easily checked and we state them without proof.

LEMMA 2.6.

(i) $\quad \sigma_{t} \sigma_{t}^{*}=\mathrm{id}, \quad \rho_{t} \rho_{t}^{*}=\mathrm{id}, \quad \sigma_{T-t}^{*} \sigma_{T-t}+\rho_{t}^{*} \rho_{t}=\mathrm{id}$, $\rho_{t} \sigma_{T-t}^{*}=0, \quad \sigma_{T-t} \rho_{t}^{*}=0$.

(ii) $\mathscr{B}(T)=S(T-t) \mathscr{B}(t) \rho_{t}+\mathscr{B}(T-t) \sigma_{T-t}$, $\mathscr{C}(T)=\sigma_{T-t}^{*} \mathscr{C}(T-t) S(t)+\rho_{t}^{*} \mathscr{C}(t)$, $\mathscr{T}(T)=\rho_{t}^{*} \mathscr{T}(t) \rho_{t}+\sigma_{T-t}^{*} \mathscr{C}(T-t) \mathscr{B}(t) \rho_{t}+\sigma_{T-t}^{*} \mathscr{T}(T-t) \sigma_{T-t}$.

(iii) $\mathscr{B}(T) \sigma_{t}^{*}=\mathscr{B}(t), \quad \mathscr{B}(T) \rho_{t}^{*}=S(T-t) \mathscr{B}(t)$, $\sigma_{T-t} \mathscr{C}(T)=\mathscr{C}(T-t) S(t), \quad \rho_{t} \mathscr{C}(T)=\mathscr{C}(t)$, $\mathscr{T}(T) \sigma_{t}^{*}=\sigma_{t}^{*} \mathscr{T}(t), \quad \rho_{t} \mathscr{T}(T)=\mathscr{T}(t) \rho_{t}$, $\sigma_{T-t} \mathscr{T}(T) \rho_{t}^{*}=\mathscr{C}(T-t) \mathscr{B}(t)$. 
It seems to be an interesting open question whether all operator families $\mathscr{B}(t)$, $\mathscr{T}(t), \mathscr{C}(t)$ with the properties of Lemma 2.6 can be represented in the above way in terms of operators $B, T_{\mu}, C$, which satisfy the hypotheses (S2)-(S4).

2.2. Boundary control systems. In this section we rewrite the SCS (2.1) into a more convenient form provided that the input operator $B \in \mathscr{L}(U, V)$ is injective and strictly unbounded that is (2.4) holds. For this purpose we introduce the space

$$
Z=\{x \in H \mid A x \in H+\operatorname{range} B\}
$$

and make it into a Hilbert space by defining

$$
\|x\|_{Z}^{2}=\|x\|_{H}^{2}+\|u\|_{U}^{2}+\|A x+B u\|_{H}^{2}
$$

where $u \in U$ is the unique input vector with $A x+B u \in H$. Then $Z \subset H$ with a continuous dense injection. Furthermore we introduce the operators $\Delta \in \mathscr{L}(Z, H)$, $\Gamma \in \mathscr{L}(Z, U)$ by defining $\Delta x=A x+B u, \Gamma x=u$ for $x \in Z$ and $u \in U$ with $A x+B u \in H$. This means that

$$
\Delta x=A x+B \Gamma x, \quad x \in Z .
$$

Finally, we define $K \in \mathscr{L}(Z, Y)$ by

$$
K x=C(\mu I-A)^{-1}(\mu x-\Delta x)+T_{\mu} \Gamma x
$$

for $x \in Z$ and $\mu \notin \sigma(A)$. Some straightforward manipulations using (2.3) show that the operator $K$ defined by (2.11) is independent of $\mu$.

As a consequence of these constructions we obtain that every solution $x(\cdot) \in$ $\mathscr{C}^{1}[0, T ; H]$ of the SCS $(2.1)$ is also a solution of the abstract boundary control system (BCS)

$$
\begin{cases}\dot{x}(t)=\Delta x(t), & t \geqslant 0, \\ \Gamma x(t)=u(t), & x(0)=x_{0}, \\ y(t)=K x(t), & \end{cases}
$$

and vice versa. The interpretation of (2.12) is that the initial value problem $\dot{x}=\Delta x$, $x(0)=x_{0}$, does not give rise to unique solutions unless the "boundary condition" $\Gamma x=u$ is also satisfied. Since equation (2.12) only makes sense if $x(t)$ is at least absolutely continuous in $H$, one might understand the solutions of the SCS (2.1) as "weak solutions" of the BCS (2.12).

Note that an analogous version of the above construction has been developed by Ho and Russell [19] for a special class of systems with a scalar input.

In Curtain and Salamon [7] the BCS (2.12) has been considered as a basic model. In fact, many systems can be formulated as a BCS of the form (2.12) in a direct way (see $\S 6$ and §7.3). From this point of view we have to assume that $Z \subset H$ are Hilbert spaces with a continuous dense injection and the operators $\Delta \in \mathscr{L}(Z, H), \Gamma \in$ $\mathscr{L}(Z, U), K \in \mathscr{L}(Z, Y)$ satisfy the following hypothesis.

$\Gamma$ is onto, $\operatorname{ker} \Gamma$ is dense in $H$, there exists a $\mu \in \mathbf{R}$ such that $\operatorname{ker}(\mu I-\Delta) \cap \operatorname{ker} \Gamma=\{0\}$ and $\mu I-\Delta$ is onto. 
At some places we also need that the output operator $K \in \mathscr{L}(Z, Y)$ is strictly unbounded (with respect to $H$ ), that is,

$$
\text { range } K^{*} \cap\left(H+\text { range } \Gamma^{*}\right)=\{0\} .
$$

Here we have identified $H$ with its dual so that $H \subset Z^{*}$ with a continuous dense injection.

REMARK 2.7. Since $\Gamma$ is onto there exists a constant $c_{0}>0$ such that for every $u \in U$ there exists an $x \in Z$ with

$$
\Gamma x=u, \quad\|x\|_{Z} \leqslant c_{0}\|u\|_{U} .
$$

In order to transform any BCS (2.12) which satisfies (B0) into an SCS of the form (2.1), we introduce the space

$$
W=\{x \in Z \mid \Gamma x=0\}
$$

and denote by $\iota: W \rightarrow Z$ the canonical injection. Then $W \subset H$ with a continuous dense injection. The operators $A \in \mathscr{L}(W, H)$ and $C \in \mathscr{L}(W, Y)$ are given by

$$
A=\Delta \iota, \quad C=K \iota .
$$

Now define $V^{*}=\mathscr{D}\left(A^{*}\right)$ as in Remark 2.1 so that $H \subset V$ with a continuous dense injection and $A$ extends to a bounded operator from $H$ into $V$. Then it follows from Remark 2.1 that $A x=\Delta x$ if and only if $\Gamma x=0$ for every $x \in Z$. This allows us to define the operators $B \in \mathscr{L}(U, V)$ and $T_{\mu} \in \mathscr{L}(U, Y)$ for $\mu \notin \sigma(A)$ as follows.

Given $u \in U$, choose $x \in Z$ such that $\Gamma x=u$ and define

$$
B u=\Delta x-A x, \quad T_{\mu} u=K x-C(\mu I-A)^{-1}(\mu x-\Delta x) .
$$

Then these operators are well defined, they are obviously linear and, by Remark 2.7, they are bounded. Furthermore, by definition, these operators satisfy (2.10) and (2.11).

Proposition 2.8. (i) Let the operators $A, B, C, T_{\mu}$ satisfy (S0), suppose that $B$ is injective and strictly unbounded and let $Z$ be defined by (2.8), (2.9). Then there exist unique operators $\Delta \in \mathscr{L}(Z, H), \Gamma \in \mathscr{L}(Z, U), K \in \mathscr{L}(Z, Y)$ satisfying (2.10) and (2.11). These operators also satisfy (B0), (2.15), (2.16). Furthermore, $Z=W \oplus$ range $(\mu I-A)^{-1} B$ for $\mu \notin \sigma(A)$ and

$$
\begin{gathered}
\Delta(\mu I-A)^{-1} B=\mu(\mu I-A)^{-1} B, \quad \Gamma(\mu I-A)^{-1} B=I, \\
K(\mu I-A)^{-1} B=T_{\mu} .
\end{gathered}
$$

(ii) Suppose that the operators $\Delta, \Gamma, K$ satisfy (B0) and let $W, A \in \mathscr{L}(W, H)$, $C \in \mathscr{L}(W, Y)$ be defined by (2.15), (2.16). Furthermore, let $V$ be the dual space of $V^{*}=\mathscr{D}\left(A^{*}\right)$. Then $A \in \mathscr{L}(H, V)$ and there exist unique operators $B \in \mathscr{L}(U, V)$, $T_{\mu} \in \mathscr{L}(U, Y), \mu \notin \sigma(A)$, satisfying (2.10) and (2.11). These operators also satisfy (S0) and $B$ is injective and strictly unbounded. Finally, $Z$ is given by (2.8) and the norm on $Z$ is equivalent to the one defined by (2.9).

(iii) Suppose that the spaces $W, H, V, Z$ and the operators $A, B, C, T_{\mu}, \Delta, \Gamma, K$ satisfy (S0), (B0) (2.8), (2.10), (2.11), (2.15), (2.16). Then every solution $x(\cdot) \in$ $\mathscr{C}^{1}[0, T ; H]$ of the SCS (2.1) satisfies the BCS (2.12) and vice versa. Furthermore, $K$ is strictly unbounded and has a dense range if and only if $C$ is strictly unbounded and has a dense range. 
Proof. (i) The existence of the operators $\Delta, \Gamma, K$ satisfying (2.10), (2.11) has been established above. Now (2.15) follows from the fact that, by definition of $\Gamma, \Gamma x=0$ if and only if $A x \in H$ which is equivalent to $x \in W$ (Remark 2.1). Furthermore, (2.16) follows from (2.15), (2.10) and (2.11). The equation

$$
A(\mu I-A)^{-1} B u+B u=\mu(\mu I-A)^{-1} B u \in H
$$

for $u \in U$ shows that $\operatorname{range}(\mu I-A)^{-1} B \subset Z$ and that (2.17) holds. (2.18) is a consequence of (2.17) and (2.11). Since $B$ is strictly unbounded, we get $W \cap$ range $(\mu I-A)^{-1} B=\{0\}$, and $Z=W \oplus \operatorname{range}(\mu I-A)^{-1} B$ follows from the identity

$$
x=(\mu I-A)^{-1}(\mu x-\Delta x)+(\mu I-A)^{-1} B \Gamma x
$$

for $x \in Z$. Finally, it follows from (2.17) that $\Gamma$ is onto.

(ii) Now let $\Delta, \Gamma, K$ be given and let $W, V, A, B, C, T_{\mu}$ be defined as above. Then $B$ is injective and strictly unbounded since $B u \in H$ and $\Gamma x=u$ imply that $A x=\Delta x-B \Gamma x \in H$ and hence $x \in W=\operatorname{ker} \Gamma$ (Remark 2.1). Now we show that $Z$ is given by (2.8). If $x \in Z$ then $A x=\Delta x-B \Gamma x \in H+$ range $B$. Conversely, if $A x+B u \in H$ for some $u \in U$ and if $\Gamma z=u, z \in Z$, then $A(x-z)=A x+B u-$ $\Delta z \in H$ and hence $x-z \in W$ which implies that $x \in Z$.

In order to establish the equivalence of the norms on $Z$, let $x \in Z$ be given and chose $z \in Z$ such that $\Gamma z=\Gamma x$ and $\|z\|_{Z} \leqslant c_{0}\|\Gamma x\|_{U}$ (Remark 2.7). Then for $\mu \in R \backslash \sigma(A)$

$$
\begin{aligned}
\|x\|_{Z} \leqslant & \|z\|_{Z}+\left\|(\mu I-A)^{-1}(\mu I-\Delta)(x-z)\right\|_{Z} \\
\leqslant & c_{0}\left[1+\left\|(\mu I-A)^{-1}(\mu I-\Delta)\right\|\right]\|\Gamma x\|_{U} \\
& +\left\|(\mu I-A)^{-1}\right\|\left[|\mu|\|x\|_{H}+\|\Delta x\|_{H}\right] \\
\leqslant & c\left[\|x\|_{H}+\|\Gamma x\|_{U}+\|\Delta x\|_{H}\right] .
\end{aligned}
$$

(iii) For the proof of statement (iii) it is convenient to identify $W^{*}$ with $Z^{*} /$ range $\Gamma^{*}$ so that $\iota^{*}: Z^{*} \rightarrow W^{*}$ is the canonical projection. Moreover, note that range $\Gamma^{*} \cap H=\{0\}$ since $\operatorname{ker} \Gamma$ is dense in $H$. This allows us to identify $x \in H$ with $\iota^{*} x=x+\operatorname{range} \Gamma^{*} \in W^{*}$.

Now suppose that $K$ is strictly unbounded and has a dense range. Then $C^{*} y=$ $\iota^{*} K^{*} y \in \iota^{*} H$ implies that $K^{*} y \in H+\operatorname{range} \Gamma^{*}$ and hence $y=0$. Therefore $C$ is strictly unbounded and has a dense range. Conversely suppose that $C$ is strictly unbounded and has a dense range. Then $K^{*} y \in H+$ range $\Gamma^{*}$ implies that $C^{*} y=$ $\iota^{*} K^{*} y \in \iota^{*} H$ and hence $y=0$. Therefore $K$ is strictly unbounded and has a dense range.

Suppose that the space $W, H, V, Z$ and the operators $A, B, C, T_{\mu}, \Delta, \Gamma, K$ satisfy (S0), (B0), (2.8), (2.10), (2.11), (2.15), (2.16). Then equation (2.17) shows that $(\mu I-A)^{-1} B: U \rightarrow Z$ is the solution operator of the abstract elliptic problem

$$
\Delta x=\mu x, \quad \Gamma x=u .
$$


The next hypothesis is related to the homogeneous equation (2.12).

For every $x_{0} \in Z$ with $\Gamma x_{0}=0$ there exists a unique solution

$$
\begin{aligned}
& x(0)=x\left(\cdot ; x_{0}, 0\right) \in \mathscr{C}[0, T ; Z] \cap \mathscr{C}^{1}[0, T ; H] \text { of } \dot{x}=\Delta x, \Gamma x \\
& =0, x(0)=x_{0}, \text { depending continuouly on } x_{0} \in Z .
\end{aligned}
$$

The implications of this hypothesis for the inhomogeneous equation are summarized in the Corollary below which follows immediately from Proposition 2.8 together with Lemma 2.3 and a result in Phillips [33].

COROllary 2.9. Let (B0) and (B1) be satisfied. Then for every $x_{0} \in Z$ and every $u(\cdot) \in W^{2,2}[0, T ; U]$ with $\Gamma x_{0}=u(0)$ there exists a unique solution $x(\cdot)=$ $x\left(\cdot ; x_{0}, u\right) \in \mathscr{C}[0, T ; Z] \cap \mathscr{C}^{1}[0, T ; H]$ of $(2.12)$. This solution depends continuously on $x_{0} \in Z$ and $\dot{u}(\cdot) \in W^{1,2}[0, T ; U]$.

The next hypotheses are related to the input/state, the state/output and the input/output relationship of the BCS (2.12).

For all $x_{0} \in Z, u(\cdot) \in W^{1,2}[0, T ; U]$ with $\Gamma x_{0}=u(0)$ there

$$
\text { exists a unique solution } x(\cdot)=x\left(\cdot ; x_{0}, u\right) \in \mathscr{C}[0, T ; Z] \cap
$$
$\mathscr{C}^{1}[0, T ; H]$ of the BCS $(2.12)$ depending continuously on $x_{0} \in Z$ and $\dot{u}(\cdot) \in L^{2}[0, T ; U]$.

(B1) is satisfied and there exists a constant $c>0$ such that the following inequality holds for every $x_{0} \in Z$ with $\Gamma x_{0}=0$.

$$
\int_{0}^{T}\left\|K x\left(t ; x_{0}, 0\right)\right\|_{Y}^{2} d t \leqslant c\left\|x_{0}\right\|_{H}^{2} .
$$

(B1) is satisfied and there exists a constant $c>0$ such that (B4) the following inequality holds for every $u(\cdot) \in W^{2,2}[0, t ; u]$ with $u(0)=0$.

$$
\int_{0}^{T}\|y(t ; 0, u)\|_{Y}^{2} d t \leqslant c \int_{0}^{T}\|u(t)\|_{U}^{2} d t
$$

where $y(t ; 0, u)=K x(t ; 0, u)$ with $x(\cdot ; 0, u) \in \mathscr{C}[0, T ; Z] \cap$ $\mathscr{C}^{1}[0, T ; H]$ as in Corollary 2.9 .

DEFINITION 2.10. The BCS (2.12) is said to be wellposed if the hypotheses (B0)-(B4) are satisfied.

Proposition 2.11. Suppose that the spaces $W, H, V, Z$ and the operators $A, B, C$, $T_{\mu}, \Delta, \Gamma, K$ satisfy (S0), (B0) (2.8), (2.10), (2.11), (2.15), (2.16). Then the SCS (2.1) satisfies hypothesis $(\mathrm{S} k)$ if and only if the BCS (2.12) satisfies hypothesis $(\mathrm{B} k)$ for $k=1,2,3,4$.

Proof. The equivalence of (S1) and (B1) follows from Phillips [33]. Furthermore, it follows from Lemma 2.5 and Proposition 2.8 that (S2) implies (B2). In fact, given $x_{0} \in z$ and $u(\cdot) \in W^{1,2}[0, T ; H]$ with $\Gamma x_{0}=u(0)$, we get $A x_{0}+B u(0)=\Delta x_{0} \in H$ and hence the function $x(\cdot)=x\left(\cdot ; x_{0}, u\right) \in \mathscr{C}^{1}[0, T ; H]$ defined by (2.6) satisfies (2.7) (Lemma 2.5). Therefore $x(\cdot)$ is the unique solution of the BCS (2.12). Since $A x(t)+B u(t)=\dot{x}(t) \in H$ it follows from (2.19) that $x(\cdot) \in \mathscr{C}[0, T ; Z]$. The continuous dependence follows easily from (2.6) and (2.7) together with (2.19). 
Conversely, suppose that (B2) is satisfied, let $v(\cdot) \in W^{1,2}[0, T ; U]$ and define

$$
x(t)=\int_{0}^{t} S(t-s) B u(s) d s, \quad u(s)=\int_{0}^{s} v(\tau) d \tau .
$$

Then $x(\cdot) \in \mathscr{C}^{1}[0, T ; H]$ and

$$
A x(t)+B u(t)=\dot{x}(t)=\int_{0}^{t} S(t-s) B v(s) d s \in H .
$$

(Lemma 2.3). By (2.19), this implies $x(\cdot) \in \mathscr{C}[0, T ; Z]$ and, by $(2.10), \dot{x}(t)=\Delta x(t)$, $\Gamma x(t)=u(t)$. Hence, by (B2), there exists a constant $c>0$ such that

$$
\left\|\int_{0}^{T} S(T-s) B v(s) d s\right\|_{H}=\|\dot{x}(T)\|_{H} \leqslant c\|v(\cdot)\|_{L^{2}[0, T ; U]} .
$$

This proves $(\mathrm{S} 2)$.

The equivalence of (S3) and (B3) is trivial. So is the equivalence of (S4) and (B4).

Note that the above proof for the equivalence of (B2) and (S2) has already been presented in Curtain and Salamon [7]. We have included the proof for the purpose of completeness.

2.3. Point observation processes. In this section we consider the case that the output operator $C \in \mathscr{L}(W, Y)$ of the SCS (2.1) has a dense range and is strictly unbounded, thi $\mathrm{t}$ is (2.5) holds. in this situation there is another way of rewriting the SCS (2.1) and it can be done by means of a procedure which is dual to the one described in the previous section.

We introduce the space

$$
X^{*}=\left\{x \in H \mid A^{*} x \in H+\operatorname{range} C^{*}\right\}
$$

and make it into a Hilbert space by defining

$$
\|x\|_{X^{*}}^{2}=\|x\|_{H}^{2}+\|y\|_{Y}^{2}+\left\|A^{*} x+C^{*} y\right\|_{H}^{2}
$$

where $y \in Y$ is the unique vector with $A^{*} x+C^{*} y \in H$. Identifying $H$ with its dual, we obtain $X^{*} \subset H \subset X$ with continuous, dense injections. Furthermore, there exist unique operators $\Lambda \in \mathscr{L}(H, X), \Omega \in \mathscr{L}(Y, X)$ satisfying $A^{*} x=\Lambda^{*} x-C^{*} \Omega^{*} x$ for $x \in X^{*}$ and hence

$$
\Lambda x=A x+\Omega C x, \quad x \in W .
$$

Finally, we define $G \in \mathscr{L}(U, X)$ by

$$
G u=(\mu I-\Lambda)(\mu I-A)^{-1} B u+\Omega T_{\mu} u
$$

for $u \in U$ and $\mu \notin \sigma(A)$. Then some straightforward manipulations involving (2.3) and (2.23) show that the operator $G$ defined by (2.24) is independent of $\mu$.

LeMma 2.12. Suppose that the spaces $W, H, V, X$ and the operators $A, B, C, T_{\mu}$, $\Lambda, \Omega, G$ satisfy (S0), (2.21), (2.23), (2.24) and that $C$ has a dense range and is strictly unbounded. Let $x \in H, u \in U, y \in Y$ be given. Then $\Lambda x+G u-\Omega y \in H$ if and only if $A x+B u \in H$ and

$$
y=C(\mu I-A)^{-1}(\mu x-A x-B u)+T_{\mu} u .
$$


Furthermore, if $\Lambda x+G u-\Omega y \in H$, then

$$
A x+B u+\Omega y=\Lambda x+G u .
$$

Proof. Suppose that $A x+B u \in H$ and $y \in Y$ is given by (2.25). Then

$$
\begin{aligned}
\Omega y & =\Omega C(\mu I-A)^{-1}(\mu x-A x-B u)+\Omega T_{\mu} u \\
& =(\Lambda-\mu I)(\mu I-A)^{-1}(\mu x-A x-B u)+\Omega T_{\mu} u+\mu x-A x-B u \\
& =\Lambda x+G u-A x-B u .
\end{aligned}
$$

In order to prove the converse implication, let us first consider the case $u=0$ and note that $z \in \mathscr{D}\left(A^{*}\right)$ if and only if $A^{*} z \in H$ (Remark 2.1) which, by definition of $\Omega$, is equivalent to $z \in X^{*}, \Omega^{*} z=0$. Hence $\Lambda x-\Omega y \in H$ implies that for all $z \in \mathscr{D}\left(A^{*}\right)$

$$
\left\langle A^{*} z, x\right\rangle=\left\langle\Lambda^{*} z, x\right\rangle-\left\langle\Omega^{*} z, y\right\rangle=\langle z, \Lambda x-\Omega y\rangle
$$

and therefore $x \in \mathscr{D}(A)=W$. Furthermore, it follows from (2.23) that $\Omega(y-C x)$ $=\Omega y-\Lambda x+A x \in H$. Since $\Omega^{*}$ is onto and $\operatorname{ker} \Omega^{*}$ is dense in $H$ (Proposition 2.8), we obtain that $\Omega$ is injective and $H \cap$ range $\Omega=\{0\}$. Hence $y=C x$ and $A x=\Lambda x-\Omega y$.

If $\Lambda x+G u-\Omega y \in H$, then it follows from (2.24) that

$$
\Lambda\left(x-(\mu I-A)^{-1} B u\right)-\Omega\left(y-T_{\mu} u\right)=\Lambda x+G u-\Omega y-\mu(\mu I-A)^{-1} B u \in H
$$

and hence

$$
x-(\mu I-A)^{-1} B u \in W, \quad C\left(x-(\mu I-A)^{-1} B u\right)=y-T_{\mu} u .
$$

This implies (2.25), $x \in H,(\mu I-A) x-B u \in H$, and therefore $A x+B u \in H$.

The previous lemma shows that every solution $x(\cdot) \in \mathscr{C}^{1}[0, T ; H]$ of the SCS (2.1) also satisfies the abstract point observation process (POP)

$$
\dot{x}(t)+\Omega y(t)=\Lambda x(t)+G u(t), \quad t \geqslant 0, x(0)=x_{0}
$$

and vice versa. In (2.27) $y \in Y$ has to be understood as the output and $u \in U$ as the input of the system. The interpretation of (2.27) is that the initial value problem $\dot{x}=\Lambda x, x(0)=x_{0}$, does not have a solution in general and has to be replaced by the differential inclusion $\dot{x}-\Lambda x \in$ range $\Omega$. It is important to note that $\Omega$ is boundedly invertible on its range so that the output of the system can be described by the action of an inverse of $\Omega$ on $\dot{x}-\Lambda x$.

Again we might have considered the POP (2.27) as our basic model where $H \subset X$ are Hilbert spaces with a continuous dense injection and the operators $\Lambda \in \mathscr{L}(H, X)$, $\Omega \in \mathscr{L}(Y, X), G \in \mathscr{L}(U, X)$ satisfy the following hypothesis.

$\Omega$ is injective and has a closed range, range $\Omega \cap H=\{0\}$, there exists a $\mu \in R$ such that $X=\operatorname{range}(\mu I-\Lambda) \oplus$ range $\Omega$ and $\mu I-\Lambda$ is injective.

At some places we also need that the input operator $G \in \mathscr{L}(U, X)$ is strictly unbounded (with respect to $H$ ) that is

$$
\text { range } G \cap(H+\text { range } \Omega)=\{0\} \text {. }
$$


In order to transform any POP (2.27) which satisfies hypothesis (P0) into an SCS of the form (2.1), we introduce the space

$$
V=X / \text { range } \Omega
$$

and denote by $\pi: X \rightarrow V$ the canonical projection. Since range $\Omega \cap H=\{0\}$ we can identify every $x \in H$ with $\pi x=x+$ range $\Omega \in V$. This makes $H$ into a dense subspace of $V$ with a continuous injection. Now let us define the operators $A \in \mathscr{L}(H, V), B \in \mathscr{L}(U, V)$ by

$$
A=\pi \Lambda, \quad B=\pi G .
$$

Then hypothesis (P0) implies that $\mu I-A \in \mathscr{L}(H, V)$ is one-to-one and onto for some $\mu \in R$. Hence $A$ is a closed operator on $V$ with a nonempty resolvent set. So is its restriction to $H$ which has the domain

$$
W:=\mathscr{D}(A)=\{x \in H \mid \Lambda x \in H+\text { range } \Omega\}
$$

and is defined by $\Lambda x-A x \in$ range $\Omega$. By Remark 2.1, the adjoint of this unbounded operator $A: \mathscr{D}(A) \rightarrow H$ coincides with the adjoint of the bounded operator $A \in \mathscr{L}(H, V)$ and has the domain

$$
\mathscr{D}\left(A^{*}\right)=V^{*}=\left\{x \in X^{*} \mid \Omega^{*} x=0\right\} .
$$

In order to construct $C \in \mathscr{L}(W, Y)$, let $x \in W$ be given and choose $y=C x \in Y$ such that $\Lambda x-A x=\Omega y$. Then $C$ is well defined, linear and satisfies (2.23). Furthermore, $C$ is bounded, since $\Omega$ has a bounded inverse on its range.

In order to construct $T_{\mu} \in \mathscr{L}(U, Y)$, let $u \in U$ be given and note that, by (2.30), $\pi(\mu I-\Lambda)(\mu I-A)^{-1} B u=B u=\pi G u$. Hence there exists a $y=T_{\mu} u \in Y$ such that $\Omega y=G u-(\mu I-\Lambda)(\mu I-A)^{-1} B u$. Since $\Omega$ is injective and has a closed range, this operator $T_{\mu}$ is well defined, bounded, linear and satisfies (2.24).

The next proposition summarizes the above transformations and is the dual result of Proposition 2.8. The proof will be omitted.

Proposition 2.13. (i) Let the operators $A, B, C, T_{\mu}$ satisfy (S0), suppose that $C$ has $a$ dense range and is strictly unbounded and let $X$ be defined by (2.21), (2.22). Then there exist unique operators $\Lambda \in \mathscr{L}(H, X), \Omega \in \mathscr{L}(Y, X), G \in \mathscr{L}(U, X)$ satisfying (2.23) and (2.24). These operators also satisfy (P0), (2.29)-(2.32). Furthermore, for $\mu \notin \sigma(A)$, the operator $C_{\mu}=C(\mu I-A)^{-1}: H \rightarrow Y$ extends to a bounded linear operator from $X$ into $Y$ and

$$
C_{\mu} \Lambda=\mu C(\mu I-A)^{-1}, \quad C_{\mu} \Omega=I, \quad C_{\mu} G=T_{\mu} .
$$

(ii) Suppose that the operators $\Lambda, \Omega, G$ satisfy (P0) and let $V, A \in \mathscr{L}(H, V)$, $B \in \mathscr{L}(U, V)$ be defined by(2.29), (2.30). Then the domain $W=\mathscr{D}(A)$ of $A$ in $H$ is given by (2.31). Furthermore, there exist unique operators $C \in \mathscr{L}(W, Y), T_{\mu} \in$ $\mathscr{L}(U, Y), \mu \notin \sigma(A)$, satisfying (2.23) and (2.24). These operators also satisfy (S0) and $C$ has a dense range and is strictly unbounded. Finally, $X^{*}$ is given by (2.21) and the norm on $X^{*}$ is equivalent to the one defined by (2.22).

(iii) Suppose that the spaces $W, H, V, X$ and the operators $A, B, C, T_{\mu}, \Lambda, \Omega, G$ satisfy (S0), (P0), (2.21), (2.23), (2.24), (2.29)-(2.32). Then every solution $x(\cdot) \in$ $\mathscr{C}^{1}[0, T ; H]$ of the SCS (2.1) satisfies the POP (2.27) and vice versa. Furthermore, $G$ is injective and strictly unbounded if and only if $B$ is injective and strictly unbounded. 
REMARK 2.14. If the SCS (2.1) and the POP (2.27) are related as above, then the map $\varphi_{\mu}: V \times Y \rightarrow X, \mu \notin \sigma(A)$, defined by $\varphi_{\mu}(x, y)=(\mu I-\Lambda)(\mu I-A)^{-1} x+\Omega y$ is an isomorphism with the properties

$$
\begin{gathered}
x=\varphi_{\mu}\left(x, C(\mu I-A)^{-1} x\right), \quad \Lambda x=\varphi_{\mu}\left(A x, \mu C(\mu I-A)^{-1} x\right), \quad x \in H, \\
\Omega y=\varphi_{\mu}(0, y), \quad G u=\varphi_{\mu}\left(B u, T_{\mu} u\right), \quad y \in Y, u \in U .
\end{gathered}
$$

This suggests an alternative procedure for transforming the SCS (2.1) into a POP of the form (2.27).

The next hypothesis is related to the homogeneous equation (2.27).

For every $x_{0} \in H$ with $\Lambda x_{0} \in H+$ range $\Omega$ there exists a unique solution $x(\cdot)=x\left(\cdot ; x_{0}, 0\right) \in \mathscr{C}^{1}[0, T ; H]$ of the initial value problem $\dot{x}-\Lambda x \in$ range $\Omega, x(0)=x_{0}$. In $\mathscr{C}[0, T ; H]$ this solution depends continuously on $x_{0} \in H$.

The implications of this hypothesis for the inhomogeneous equation are summarized in the corollary below which follows immediately from Proposition 2.13 together with Lemma 2.3 and a result in Phillips [33].

COROllaRY 2.15. Let (P0) and (P1) be satisfied. Then for every $x_{0} \in H$ and every $u(\cdot) \in W^{2,2}[0, T ; U]$ with $\Lambda x_{0}+G u(0) \in H+$ range $\Omega$ there exists a unique solution pair $y(\cdot)=y\left(\cdot ; x_{0}, u\right) \in \mathscr{C}[0, T ; Y], x(\cdot)=x\left(\cdot ; x_{0}, u\right) \in \mathscr{C}^{1}[0, T ; H]$ of (2.27). This solution pair depends continuously on $x_{0} \in H, \dot{u}(\cdot) \in W^{1,2}[0, T ; U]$ and the H-component of $\Lambda x_{0}+G u(0)$. Furthermore, $x\left(\cdot ; x_{0}, u\right)$ depends in $\mathscr{C}[0, T ; H]$ continuously on $x_{0} \in H$ and $u(\cdot) \in W^{1,2}[0, T ; U]$.

The following hypotheses are related to the input/state, the state/output and input/output behavior of the POP (2.27).

For every $x_{0} \in H$ and every $u(\cdot) \in W^{1,2}[0, T ; U]$ with $\Lambda x_{0}$ $+G u(0) \in H+$ range $\Omega$ there exists a unique solution pair (P2) $\quad y(\cdot)=y\left(\cdot ; x_{0}, u\right) \in \mathscr{C}[0, T ; Y], \quad x(\cdot)=x\left(\cdot ; x_{0}, u\right) \in$ $\mathscr{C}^{1}[0, T ; H]$ of $(2.27)$. This solution pair depends continuously on $x_{0} \in H, u(\cdot) \in W^{1,2}[0, T ; H]$ and on the $H$-component of $\Lambda x_{0}+G u(0)$.

For every $x_{0} \in H$ there exists a unique solution pair $x(\cdot)=$ (P3) $\quad x\left(\cdot ; x_{0}, 0\right) \in \mathscr{C}[0, T ; H] \cap W^{1,2}[0, T ; X]$ and $y(\cdot)=$ $y\left(\cdot ; x_{0}, 0\right) \in L^{2}[0, T ; H]$ of $(2.27)$ with $u(\cdot)=0$. This solution pair depends continuously on $x_{0} \in H$.

(P1) is satisfied and there exists a constant $c>0$ such that for (P4) every $u(\cdot) \in W^{2,2}[0, T ; U]$ with $U(0)=0$ the following inequality holds

$$
\int_{0}^{T}\|y(t ; 0, u)\|_{Y}^{2} d t \leqslant c \int_{0}^{T}\|u(t)\|_{U}^{2} d t .
$$


DEFINITION 2.16. The POP (2.27) is said to be wellposed if (P0)-(P4) are satisfied.

Proposition 2.17. Suppose that the spaces $W, H, V, X$ and the operators $A, B, C$, $T_{\mu}, \Lambda, \Omega, G$ satisfy (S0), (P0), (2.21), (2.23), (2.24), (2.29-32). Then the following statements holds.

(i) The SCS (2.1) satisfies hypothesis (Sk) if and only if the POP (2.27) satisfies hypothesis $(\mathrm{P} k)$ for $k=1,2,3,4$.

(ii) The POP (2.27) is wellposed in the sense of Definition 2.16 if and only if for every $x_{0} \in H$ and every $u(\cdot) \in L^{2}[0, T ; U]$ there exists a unique solution pair $x(\cdot)=$ $x\left(\cdot ; x_{0}, u\right) \in \mathscr{C}[0, T ; H] \cap W^{1,2}[0, T ; X]$ and $y(\cdot)=y\left(\cdot ; x_{0}, u\right) \in L^{2}[0, T ; Y]$ of (2.27) depending continuously on $x_{0} \in H$ and $u(\cdot) \in L^{2}[0, T ; U]$.

Proof. The proof of statement (i) is analogous to that of Proposition 2.11 and will be omitted.

In order to prove statement (ii) let us first assume that the POP (2.27) is wellposed and choose $x_{0} \in H, u(\cdot) \in W^{1,2}[0, T ; U]$ with $\Lambda x_{0}+G u(0) \in H+$ range $\Omega$. Then it follows from statement (i) that the SCS (2.1) is wellposed and we can denote by $x\left(\cdot ; x_{0}, u\right) \in \mathscr{C}^{1}[0, T ; H]$ and $y\left(\cdot ; x_{0}, u\right) \in W^{1,2}[0, T ; Y]$ the corresponding solution and output of (2.1). Furthermore, it follows from Lemma 2.12 that

$$
x\left(t ; x_{0}, u\right)-x_{0}=\int_{0}^{T}\left[\Lambda x\left(s ; x_{0}, u\right)+G u(s)-\Omega y\left(s ; x_{0}, u\right)\right] d s
$$

for $0 \leqslant t \leqslant T$. Since both sides of this equation depend continuously on $x_{0} \in H$ and $u(\cdot) \in L^{2}[0, T ; U]$ we obtain that $x\left(\cdot ; x_{0}, u\right) \in \mathscr{C}[0, T ; H] \cap W^{1,2}[0, T ; X]$ and $y\left(\cdot ; x_{0}, u\right) \in L^{2}[0, T ; Y]$ satisfy $(2.27)$ for every $x_{0} \in H$ and every $u(\cdot) \in L^{2}[0, T ; U]$. The uniqueness follows from the fact that every solution $x(\cdot) \in \mathscr{C}[0, T ; H] \cap$ $W^{1,2}[0, T ; X]$ of $(2.27)$ also satisfies $(2.1 ; 1)$ in $V=X /$ range $\Omega$. This fact also proves the converse implication via the wellposedness of the SCS (2.1).

Note that in the case of the SCS (2.1) and of the BCS (2.12) we have to assume that $x\left(\cdot ; x_{0}, u\right) \in \mathscr{C}^{1}[0, T ; H]$ in order to give a meaning to the expression $y\left(t ; x_{0}, u\right)$ in a strong sense. The previous proposition shows that for the POP (2.27) both $x\left(t ; x_{0}, u\right)$ and $y\left(t ; x_{0}, u\right)$ have a well-defined meaning as strong solutions for arbitrary $x_{0} \in H$ and $u(\cdot) \in L^{2}[0, T ; U]$.

The relation between the various spaces and operators can be summarized by the following diagram in which the vertical sequences are exact.

$$
\begin{aligned}
& \text { Y } \\
& \downarrow \Omega \\
& \Lambda \nearrow \quad \begin{array}{ll}
X \\
\downarrow \pi & \nwarrow G
\end{array} \\
& Y \stackrel{C}{\leftarrow} W \stackrel{A}{\rightarrow} H \stackrel{A}{\rightarrow} \quad V \quad \underset{B}{\leftarrow} U \\
& K \nwarrow \quad \downarrow \iota \nearrow \Delta \\
& Z \\
& \downarrow
\end{aligned}
$$


Finally, we point out that the above results can be combined to transform the POP (2.27) directly into a BCS of the form (2.12) and vice versa. These transformations are summarized in the proposition below. Its proof follows from the earlier results of this section and will be omitted.

PROPOSITION 2.18. (i) Let the operators $\Lambda, \Omega, G$ satisfy ( $\mathrm{P} 0$ ), suppose that $G$ is injective and strictly unbounded and define $Z \subset H$ by

$$
\begin{gathered}
Z=\{x \in H \mid \Lambda x \in H+\text { range } G+\text { range } \Omega\}, \\
\|x\|_{Z}^{2}=\|x\|_{H}^{2}+\|u\|_{U}^{2}+\|\Lambda x+G u-\Omega y\|_{H}^{2}
\end{gathered}
$$

for $x \in Z, u \in U, y \in Y$ with $\Lambda x+G u-\Omega y \in H$. Then $Z$ is a Hilbert space with a continuous, dense injection into $H$ and there exist unique operators $\Delta \in \mathscr{L}(Z, H)$, $\Gamma \in \mathscr{L}(Z, U), K \in \mathscr{L}(Z, Y)$ such that

$$
\Delta x+\Omega K x=\Lambda x+G \Gamma x, \quad x \in Z .
$$

These operators also satisfy (B0) and $K$ has a dense range and is strictly unbounded. Furthermore $X^{*} \subset H$ is given by

$$
X^{*}=\left\{x \in H \mid \Delta^{*} x \in H+\operatorname{range} K^{*}+\operatorname{range} \Gamma^{*}\right\}
$$

and the norm on $X^{*}$ is equivalent to the one defined by

$$
\|x\|_{X^{*}}^{2}=\|x\|_{H}^{2}+\|y\|_{Y}^{2}+\left\|\Delta^{*} x+K^{*} y-\Gamma^{*} u\right\|_{U}^{2}
$$

for $x \in X^{*}, y \in Y, u \in U$ with $\Delta^{*} x+K^{*} y-\Gamma^{*} u \in H$.

(ii) Let the operators $\Delta, \Gamma, K$ satisfy (B0), suppose that $K$ has a dense range and is strictly unbounded and let $X^{*}$ be defined by (2.37), (2.38). Then $X^{*}$ is a Hilbert space with a continuous, dense injection into $H$ and there exist unique operators $\Lambda \in(H, X)$, $\Omega \in(Y, X), G \in(U, X)$ satisfying (2.36). These operators also satisfy $(\mathrm{P} 0)$ and $G$ is injective and strictly unbounded. Furthermore, $Z \subset H$ is given by (2.34) and the norm on $Z$ is equivalent to the one defined by (2.35).

(iii) If the spaces $Z \subset H \subset X$ and the operators, $\Lambda, \Omega, G, \Delta, \Gamma, K$ satisfy ( $\mathrm{P} 0$ ), (B0), (2.34), (2.36), (2.37), then every solution $x(\cdot) \in \mathscr{C}^{1}[0, T ; H]$ of the POP (2.27) also satisfies the BCS (2.12) and vice versa. Furthermore, hypothesis $(\mathrm{P} k)$ is equivalent to hypothesis $(\mathrm{B} k)$ for $k=1,2,3,4$.

3. Duality. Consider the SCS

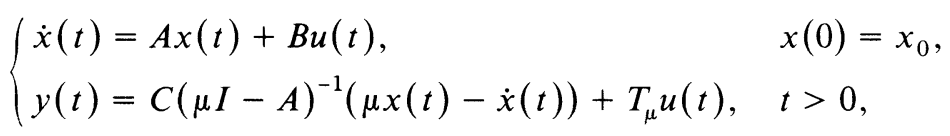

where $W \subset H \subset V$ with continuous, dense injections and the operators $A \in$ $\mathscr{L}(W, H) \cap \mathscr{L}(H, V), B \in \mathscr{L}(U, V), C \in \mathscr{L}(W, Y), T_{\mu} \in \mathscr{L}(U, Y)$ satisfy hypothesis (S0). Identifying the spaces $H, U, Y$ with their respective duals we obtain $V^{*} \subset H \subset W^{*}$ with continuous, dense injections and the adjoint operators $A^{*} \in$ $\mathscr{L}\left(V^{*}, H\right) \cap \mathscr{L}\left(H, W^{*}\right), B^{*} \in \mathscr{L}\left(V^{*}, U\right), C^{*} \in \mathscr{L}\left(Y, W^{*}\right), T_{\mu}^{*} \in \mathscr{L}(Y, U)$ also satisfy hypothesis (S0). For some purposes it is convenient to write the dual system 
in the time reverse form

$$
\begin{cases}\dot{z}(s)=-A^{*} z(s)-C^{*} v(s), & z(T)=z_{1}, \\ w(s)=B^{*}\left(\mu I-A^{*}\right)^{-1}(\mu z(s)+\dot{z}(s))+T_{\mu} v(s), & s \leqslant T,\end{cases}
$$

where $v \in Y$ is the input and $w \in U$ is the output. For every $z_{1} \in H$ and every $v \in L^{2}[0, T ; Y]$ the solution of (3.2) is given by

$$
z\left(s ; z_{1}, v\right)=S^{*}(T-s) z_{1}+\int_{s}^{T} S^{*}(t-s) C^{*} v(t) d t, \quad s \leqslant T .
$$

Lemma 2.3 shows that $z\left(\cdot ; z_{1}, v\right) \in \mathscr{C}^{1}[0, T ; H]$ if $v(\cdot) \in W^{2,2}[0, T ; Y]$ and $A^{*} z_{1}+$ $C^{*} v(T) \in H$ and that in this case $\dot{z}\left(s ; z_{1}, v\right)=z\left(s ;-A^{*} z_{1}-C^{*} v(T), \dot{v}\right)$. Whenever $z(\cdot)=z\left(\cdot ; z_{1}, v\right) \in W^{1,2}[0, T ; H]$ we denote by $w\left(\cdot ; z_{1}, v\right)$ the corresponding output of (3.2). We consider the following hypotheses for the SCS (3.2).

(S0*) The operator $\mu I-A^{*}: V^{*} \rightarrow H$ is boundedly invertible for some $\mu \in R, W=\mathscr{D}(A)$, and

$$
T_{\mu}^{*}-T_{\lambda}^{*}=(\lambda-\mu) B^{*}\left(\mu I-A^{*}\right)^{-1}\left(\lambda I-A^{*}\right)^{-1} C^{*}
$$

for all $\lambda, \mu \notin \sigma(A)$.

$\left(\mathrm{S} 1^{*}\right)$ The operator $A^{*}: \mathscr{D}\left(A^{*}\right)=V^{*} \rightarrow H$ is the infinitesimal generator of a strongly continuous semigroup $S^{*}(t) \in \mathscr{L}(H)$.

$\left(\mathrm{S} 2^{*}\right) \quad\left(\mathrm{S} 1^{*}\right)$ is satisfied and there exists a constant $c>0$ such that the following inequality holds for all $x \in V^{*}$ :

$$
\left\|B^{*} S *(T-\cdot) x\right\|_{L^{2}[0, T ; U]} \leqslant c\|x\|_{H} .
$$

$\left(\mathrm{S} 1^{*}\right)$ is satisfied and there exists a constant $c>0$ such that the following inequality holds for all $v(\cdot) \in W^{1,2}[0, T ; Y]$ :

$$
\left\|\int_{0}^{T} S^{*}(t) C^{*} v(t) d t\right\|_{H} \leqslant c\|v(\cdot)\|_{L^{2}[0, T ; Y]} .
$$

$\left(\mathrm{S} 1^{*}\right)$ is satisfied and there exists a $c>0$ such that the $\left(\mathrm{S} 4^{*}\right) \quad$ following inequality holds for all $v(\cdot) \in W^{2,2}[0, T ; Y]$ with $v(T)=0$ :

$$
\|w(\cdot ; 0, v)\|_{L^{2}[0, T ; U]} \leqslant c\|v(\cdot)\|_{L^{2}[0, T ; Y]} .
$$

DEFINITION 3.1. The SCS (3.2) is said to be wellposed if $(\mathrm{S} 0)-(\mathrm{S} 4 *)$ are satisfied. If the SCS (3.2) is wellposed and $z_{1} \in H, v(\cdot) \in L^{2}[0, T ; Y]$ are given, we define $z(s)=z\left(s ; z_{1}, v\right)$ by (3.3) and $w(s)=w\left(s ; z_{1}, v\right)$ by continuous extension of the expression in (3.2) using ( $\mathrm{S} 2 *)$ and $\left(\mathrm{S} 4^{*}\right) . w(s)$ is said to be the weak output of the SCS (3.2).

Although the next result is strictly analogous to Lemma 2.5 it is worth being stated explicitly since it formulates the basic properties of the solutions of the SCS (3.2) in the time reverse situation and will be needed in $\S 5$. 
LEMma 3.2. Suppose that the SCS (3.2) is wellposed, let $z_{1} \in H, v(\cdot) \in L^{2}[0, T ; Y]$ be given and let $z(s)=z\left(s ; z_{1}, v\right)$ and $w(s)=w\left(s ; z_{1}, v\right)$ be defined as above. Then the following statements holds.

(i) $z\left(\cdot ; z_{1}, v\right) \in \mathscr{C}[0, T ; H] \cap W^{1,2}\left[0, T ; W^{*}\right]$ satisfies (3.2) for almost every $s \in$ $[0, T]$.

(ii) If $z\left(\cdot ; z_{1}, v\right) \in W^{1,2}[0, T ; H]$ then $w\left(s ; z_{1}, v\right)$ is given by (3.2) for almost every $s \in[0, T]$.

(iii) If $v(\cdot) \in W^{1,2}[0, T ; Y]$ and $A^{*} z_{1}+C^{*} v(T) \in H$, then $z\left(\cdot ; z_{1}, v\right) \in$ $\mathscr{C}^{1}[0, T ; H], w\left(\cdot ; z_{1}, v\right) \in W^{1,2}[0, T ; U]$ satisfy $\dot{z}\left(s ; z_{1}, v\right)=z\left(s ;-A^{*} z_{1}-C^{*} v(T), \dot{v}\right)$ and $\dot{w}\left(s ; z_{1}, v\right)=w\left(s ;-A^{*} z_{1}-C^{*} v(T), \dot{v}\right)$ for (almost) every $s \in[0, T]$.

Our basic duality theorem is the following.

THEOREM 3.3. (i) The SCS (3.1) satisfies hypothesis ( $\mathrm{S} k$ ) if and only if the dual SCS (3.2) satisfies hypothesis $\left(\mathrm{S}^{*}\right)$ for $k=0,1,2,3,4$.

(ii) Suppose that the SCS (3.1) satisfies hypothesis (S0), that $u(\cdot) \in L^{2}[0, T ; U]$, $x(\cdot) \in W^{1,2}[0, T ; H], y(\cdot) \in L^{2}[0, T ; Y]$ satisfy $(3.1)$ and that $v(\cdot) \in L^{2}[0, T ; Y]$, $z(\cdot) \in W^{1,2}[0, T ; H], w(\cdot) \in L^{2}[0, T ; U]$ satisfy (3.2). Then

$$
\begin{aligned}
\langle z(t), x(t)\rangle_{H}-\langle z(s), x(s)\rangle_{H} & =\int_{s}^{t}\langle w(\tau), u(\tau)\rangle_{U} d \tau \\
& -\int_{s}^{t}\langle v(\tau), y(\tau)\rangle_{Y} d \tau, \quad 0 \leqslant s \leqslant t \leqslant T .
\end{aligned}
$$

Proof. The equivalence of ( $\mathrm{S} 0)$ and $\left(\mathrm{S} 0^{*}\right)$ follows from Remark 2.1, the equivalence of $(\mathrm{S} 1)$ and $\left(\mathrm{S} 1^{*}\right)$ is a well-known result in semigroup theory and the equivalence of $(\mathrm{S} 2)$ and $\left(\mathrm{S} 22^{*}\right)$ as well as $(\mathrm{S} 3)$ and $\left(\mathrm{S} 3^{*}\right)$ has been established in [40]. The equivalence of (S4) and (S4*) follows from statement (ii) together with the fact that the functions $u(\cdot) \in W^{2,2}[0, T ; U]$ with $u(0)=0$ are dense in $L^{2}[0, T ; U]$. Now let the assumptions of statement (ii) be satisfied. Then

$$
\begin{aligned}
\langle z(t), & x(t)\rangle_{H}-\langle z(s), x(s)\rangle_{H} \\
= & \int_{s}^{t}\langle\dot{z}(\tau), x(\tau)\rangle_{H} d \tau+\int_{s}^{t}\langle z(\tau), \dot{x}(\tau)\rangle_{H} d \tau \\
= & \int_{s}^{t}\left\langle\dot{z}(\tau),(\mu I-A)^{-1}(\mu x(\tau)-\dot{x}(\tau)+B u(\tau))\right\rangle_{H} d \tau \\
& +\int_{s}^{t}\left\langle\left(\mu I-A^{*}\right)^{-1}\left(\mu z(\tau)+\dot{z}(\tau)+C^{*} v(\tau)\right), \dot{x}(\tau)\right\rangle_{H} d \tau \\
= & \int_{s}^{t}\left\langle B^{*}\left(\mu I-A^{*}\right)^{-1} \dot{z}(\tau), u(\tau)\right\rangle_{U} d \tau+\int_{s}^{t}\left\langle\dot{z}(\tau), \mu(\mu I-A)^{-1} x(\tau)\right\rangle_{H} d \tau \\
& +\int_{s}^{t}\left\langle v(\tau), C(\mu I-A)^{-1} \dot{x}(\tau)\right\rangle_{Y} d \tau+\int_{s}^{t}\left\langle\mu\left(\mu I-A^{*}\right)^{-1} z(\tau), \dot{x}(\tau)\right\rangle_{H} d \tau \\
= & \int_{s}^{t}\left\langle B^{*}\left(\mu I-A^{*}\right)^{-1}(\mu z(\tau)+\dot{z}(\tau)), u(\tau)\right\rangle_{U} d \tau \\
& -\int_{s}^{t}\left\langle v(\tau), C(\mu I-A)^{-1}(\mu x(\tau)-\dot{x}(\tau))\right\rangle_{Y} d \tau \\
= & \int_{s}^{t}\langle w(\tau), u(\tau)\rangle_{U} d \tau-\int_{s}^{t}\langle v(\tau), y(\tau)\rangle_{Y} d \tau .
\end{aligned}
$$


Defining the operators $\mathscr{B}(T), \mathscr{C}(T), \mathscr{T}(T), \sigma_{t}, \rho_{t}$ as in $\S 2.1$, we obtain the following result as a straightforward consequence of Theorem 3.3.

COROLlaRY 3.4. If the SCS (3.2) is wellposed in the sense of Definition 3.1, then the following equations hold for every $z_{1} \in H$ and every $v(\cdot) \in L^{2}[0, T ; Y]$

$$
\begin{gathered}
z\left(s ; z_{1}, v\right)=S^{*}(T-s) z_{1}+\mathscr{C}^{*}(T-s) \sigma_{T-s} v, \quad 0 \leqslant s \leqslant T, \\
w\left(\cdot ; z_{1}, v\right)=\mathscr{B} *(T) z_{1}+\mathscr{T}^{*}(T) v .
\end{gathered}
$$

Let us now consider the BCS

$$
\left\{\begin{array}{l}
\dot{x}(t)=\Delta x(t), \quad x(0)=x_{0}, \\
\Gamma x(t)=u(t), \quad t \geqslant 0, \\
y(t)=K x(t)
\end{array}\right.
$$

where $Z \subset H$ with a continuous dense injection and the operators $\Delta \in \mathscr{L}(Z, H)$, $\Gamma \in \mathscr{L}(Z, U), K \in \mathscr{L}(Z, Y)$ satisfy hypothesis (B0). Then $H \subset Z^{*}$ with a continuous, dense injection and the dual operators $\Delta^{*} \in \mathscr{L}\left(H, Z^{*}\right), \Gamma^{*} \in \mathscr{L}\left(U, Z^{*}\right)$, $K^{*} \in \mathscr{L}\left(Y, Z^{*}\right)$ satisfy hypothesis (P0) with $X=Z^{*}, \Lambda=\Delta^{*}, \Omega=\Gamma^{*}, G=K^{*}$. As the dual system of the BCS (3.5) we consider the POP

$$
\dot{z}(s)-\Gamma^{*} w(s)=-\Delta^{*} z(s)-K^{*} v(s), \quad s \leqslant T, z(T)=z_{1},
$$

where $v \in Y$ is the input and $w \in U$ is the output. If the BCS (3.5) is related to the SCS (3.1) as in $\S 2.2$ then the POP (3.6) is related to the SCS (3.2) as in $\S 2.3$. This means that the following diagram commutes.

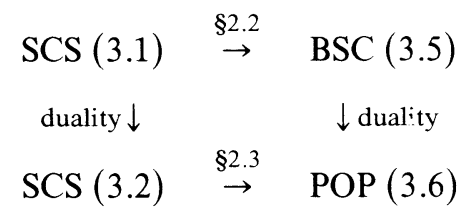

Making use of this fact we obtain the following duality relationship between the systems (3.5) and (3.6) which can also be proved directly in a straightforward way.

THEOREM 3.5. (i) The BCS (3.5) satisfies hypothesis $(\mathrm{B} k)$ if and only if the POP (3.6) satisfies hypothesis $(\mathrm{P} k)$ with $X=Z^{*}, \Lambda=\Delta^{*}, \Omega=\Gamma^{*}, G=K^{*}$ for $k=0,1,4$. Furthermore, (B2) is equivalent to $(\mathrm{P} 3)$ and $(\mathrm{B} 3)$ is equivalent to $(\mathrm{P} 2)$.

(ii) Suppose that the $B C S$ (3.5) satisfies (B0), that $u(\cdot) \in L^{2}[0, T ; U], x(\cdot) \in$ $L^{2}[0, T ; Z] \cap W^{1,2}[0, T ; H]$ and $y(\cdot) \in L^{2}[0, T ; Y]$ satisfy (3.5) and that $v(\cdot) \in$ $L^{2}[0, T ; Y], z(\cdot) \in W^{1,2}[0, T ; H]$ and $w(\cdot) \in L^{2}[0, T ; U]$ satisfy (3.6). Then (3.4) holds.

Finally, we point out that the dual system of the POP

$$
\dot{x}(t)+\Omega y(t)=\Lambda x(t)+G u(t), \quad t \geqslant 0, x(0)=x_{0},
$$

is the $\mathrm{BCS}$

$$
\begin{cases}\dot{z}(s)=-\Lambda^{*} z(s), & z(T)=z_{1}, \\ \Omega^{*} z(s)=v(s), & s \leqslant T, \\ w(s)=G^{*} z(s), & \end{cases}
$$

where $v \in Y$ is the input and $w \in U$ is the output. 
4. A perturbation result. In the finite dimensional feedback problem

$$
\dot{x}=A x+B u, \quad u=F x,
$$

the closed loop input $u_{F}(t)=F e^{(A+B F) t} x_{0}$ satisfies the integral equation

$$
u_{F}(t)=F e^{A t} x_{0}+F \int_{0}^{t} e^{A(t-s)} B u_{F}(s) d s .
$$

In finite dimensions, this equation always has a unique solution. In infinite dimensions this nice property can break down due to the unboundedness in both operators $B$ and $F$. An example for this is provided by the wave equation in $\S 7.3$. Therefore we need a condition to guarantee that the operator $I-\mathscr{T}(t)$ is boundedly invertible. Note that in our case the output operator plays the role of $F$.

From now on we will assume throughout this section that the SCS (2.1) is wellposed in the sense of Definition 2.4, that the operators $\mathscr{B}(t), \mathscr{C}(t), \mathscr{T}(t)$ are defined as in $\$ 2.1$ and that $U=Y$.

LEMma 4.1. Let $T>0$ be fixed. Then $I-\mathscr{T}(T)$ is invertible if and only if $I-\mathscr{T}(t)$ is invertible for every $t>0$.

Proof. We fix $0<t<T$ and prove that $I-\mathscr{T}(T)$ is invertible if and only if $I-\mathscr{T}(t)$ is invertible. At various places we use Lemma 2.6 without stating it explicitly.

Let us first assume that $I-\mathscr{T}(T)$ is invertible. Then $u-\mathscr{T}(t) u=0, u \in$ $L^{2}[0, t ; U]$, implies that $\sigma_{t}^{*} u=\sigma_{t}^{*} \mathscr{T}(t) u=\mathscr{T}(T) \sigma_{t}^{*} u$ and hence $\sigma_{t}^{*} u=0$ which means that $u=0$. Given $y \in L^{2}[0, t ; U]$, there exists a $\bar{u} \in L^{2}[0, T ; U]$ with $\bar{u}-$ $\mathscr{T}(T) \bar{u}=\sigma_{t}^{*} y$. This implies $\rho_{T-t} \bar{u}=\rho_{T-t} \mathscr{T}(T) \bar{u}=\mathscr{T}(T-t) \rho_{T-t} \bar{u}$ and hence $\rho_{T-t} \bar{u}=0$. Therefore $u=\sigma_{t} \bar{u}$ satisfies $\sigma_{t}^{*} u=\bar{u}$ and thus $\sigma_{t}^{*} y=\sigma_{t}^{*} u-\mathscr{T}(T) \sigma_{t}^{*} u$ $=\sigma_{t}^{*}(u-\mathscr{T}(t) u)$ which implies $y=u-\mathscr{T}(t) u$.

Conversely suppose that $I-\mathscr{T}(t)$ is invertible and w.l.o.g. $0<t<T<2 t$. Then it follows from what we just proved that $I-\mathscr{T}(T-t)$ is invertible. Assume first that $u \in L^{2}[0, T ; U]$ satisfies $u=\mathscr{T}(T) u$. Then $\rho_{t} u=\rho_{t} \mathscr{T}(T) u=\mathscr{T}(t) \rho_{t} u$ and hence $\rho_{t} u=0$ which implies $u=\sigma_{T-t}^{*} \sigma_{T-t} u$. We conclude that $\sigma_{T-t}^{*} \sigma_{T-t} u=$ $\sigma_{T-t}^{*} \mathscr{T}(T-t) \sigma_{T-t} u$ and thus $\sigma_{T-t} u=\mathscr{T}(T-t) \sigma_{T-t} u$ which means that $u=$ $\boldsymbol{\sigma}_{T-t}^{*} \boldsymbol{\sigma}_{T-t} u=0$. Secondly, let $y \in L^{2}[0, T ; U]$ be given and choose $u_{0} \in L^{2}[0, t ; U]$, $u_{1} \in L^{2}[0, T-t ; U]$ such that $u_{0}-\mathscr{T}(t) u_{0}=\rho_{t} y$ and $u_{1}-\mathscr{T}(T-t) u_{1}=\sigma_{T-t} y$ $+\mathscr{C}(T-t) \mathscr{B}(t) u_{0}$. Then it follows from Lemma 2.6 that $u=\sigma_{T-t}^{*} u_{1}+\rho_{t}^{*} u_{0}$ satisfies $u-\mathscr{T}(T) u=y$.

THEOREM 4.2. Suppose that the SCS (2.1) is wellposed in the sense of Definition 2.4, that $U=Y$ and that $I-\mathscr{T}(t) \in \mathscr{L}\left(L^{2}[0, t ; U]\right)$ is boundedly invertible for $t>0$. Then the bounded linear operators

$$
S_{F}(t)=S(t)+\mathscr{B}(t)[I-\mathscr{T}(t)]^{-1} \mathscr{C}(t) \in \mathscr{L}(H), \quad t \geqslant 0,
$$

define a strongly continuous semigroup. Moreover,

$$
S_{F}(t) x_{0}=S(t) x_{0}+\int_{0}^{t} S(t-s) B u_{F}\left(s ; x_{0}\right) d s
$$

for $x_{0} \in H$ and $t \geqslant 0$ where the closed loop input $u_{F}\left(\cdot ; x_{0}\right) \in L^{2}[0, T ; U]$ is defined by $u_{F}\left(\cdot ; x_{0}\right)=[I-\mathscr{T}(T)]^{-1} \mathscr{C}(T) x_{0}$. 
Proof. It follows from Lemma 2.6(iii) that

$$
\rho_{t}[I-\mathscr{T}(T)]^{-1} \mathscr{C}(T)=[I-\mathscr{T}(t)]^{-1} \rho_{t} \mathscr{C}(T)=[I-\mathscr{T}(t)]^{-1} \mathscr{C}(t)
$$

for $0 \leqslant t \leqslant T$. This proves equation (4.2) and the strong continuity of $S_{F}(t)$ in $H$ (Lemma 2.5(i)). It follows from Lemma 2.6(ii) that $\sigma_{T-t} \mathscr{T}(T)=\mathscr{T}(T-t) \sigma_{T-t}+$ $\mathscr{C}(T-t) \mathscr{B}(t) \rho_{t}$ and hence, by Lemma 2.6(iii),

$$
\begin{aligned}
\sigma_{T-t}[I-\mathscr{T}(T)]^{-1}= & {[I-\mathscr{T}(T-t)]^{-1} \sigma_{T-t} } \\
& +[I-\mathscr{T}(T-t)]^{-1} \mathscr{C}(T-t) \mathscr{B}(t)[I-\mathscr{T}(t)]^{-1} \rho_{t}
\end{aligned}
$$

for $0 \leqslant t \leqslant T$. Putting things together, we obtain again using Lemma 2.6(ii) and (iii)

$$
\begin{aligned}
S_{F}(T)= & S(T)+\mathscr{B}(T)[I-\mathscr{T}(T)]^{-1} \mathscr{C}(T) \\
= & S(T-t)\left[S(t)+\mathscr{B}(t) \rho_{t}[I-\mathscr{T}(T)]^{-1} \mathscr{C}(T)\right] \\
& +\mathscr{B}(T-t) \sigma_{T-t}[I-\mathscr{T}(T)]^{-1} \mathscr{C}(T) \\
= & S(T-t) S_{F}(t)+\mathscr{B}(T-t)[I-\mathscr{T}(T-t)]^{-1} \sigma_{T-t} \mathscr{C}(T) \\
& +\mathscr{B}(T-t)[I-\mathscr{T}(T-t)]^{-1} \mathscr{C}(T-t) \mathscr{B}(t)[I-\mathscr{T}(t)]^{-1} \mathscr{C}(t) \\
= & {\left[S(T-t)+\mathscr{B}(T-t)[I-\mathscr{T}(T-t)]^{-1} \mathscr{C}(T-t)\right] S_{F}(t) } \\
= & S_{F}(T-t) S_{F}(t) .
\end{aligned}
$$

This proves the theorem.

The next theorem is concerned with the properties of the infinitesimal generator $A_{F}$ of the feedback semigroup $S_{F}(t)$.

THEOREM 4.3. Let the assumptions of Theorem 4.2 be satisfied and let $W_{F} \subset H$ denote the linear subspace of all $x_{0} \in H$ for which there exists $a u_{0} \in U$ and $a$ $\mu \notin \sigma(A)$ such that

$$
A x_{0}+B u_{0} \in H, \quad u_{0}=C(\mu I-A)^{-1}\left(\mu x_{0}-A x_{0}-B u_{0}\right)+T_{\mu} u_{0} .
$$

Then the following statements hold.

(i) If $x_{0} \in W_{F}, u_{0} \in U, \mu \notin \sigma(A)$ satisfy (4.3) then $x_{0} \in \mathscr{D}\left(A_{F}\right)$ and $A_{F} x_{0}=A x_{0}$ $+B u_{0}$. Furthermore, $u_{F}\left(\cdot ; x_{0}\right) \in W^{1,2}[0, T ; U], \quad S_{F}(\cdot) x_{0} \in \mathscr{C}^{1}[0, T ; H]$ satisfy $\dot{u}_{F}\left(t ; x_{0}\right) \equiv u_{F}\left(t ; A_{F} x_{0}\right), u_{F}\left(0 ; x_{0}\right)=u_{0}$ and for $t \geqslant 0$

$$
\begin{gathered}
\frac{d}{d t} S_{F}(t) x_{0}=A S_{F}(t) x_{0}+B u_{F}\left(t ; x_{0}\right)=S_{F}(t) A_{F} x_{0}, \\
u_{F}\left(t ; x_{0}\right)=C(\mu I-A)^{-1} S_{F}(t)\left(\mu I-A_{F}\right) x_{0}+T_{\mu} u_{F}\left(t ; x_{0}\right) .
\end{gathered}
$$

(ii) If $x_{0} \in \mathscr{D}\left(A_{F}\right)$ then $S_{F}(t) x_{0} \in W_{F}$ and (4.4), (4.5) hold for all $t>0$. Furthermore, $x_{0} \in W_{F}$ if and only if $x_{0} \in \mathscr{D}\left(A_{F}\right)$ and $u_{F}\left(t ; x_{0}\right)$ is continuous at $t=0$.

(iii) $W_{F}$ is dense in $H$.

(iv) If $\left\{\left(u-T_{\mu} u, B u\right) \mid u \in U\right\}$ is a closed subspace of $U \times V$ then $\mathscr{D}\left(A_{F}\right)=W_{F}$.

(v) If $C \in \mathscr{L}(H, Y)$ and $T_{\mu}=C(\mu I-A)^{-1} B$ then $\mathscr{D}\left(A_{F}\right)=W_{F}$. 
Proof. Suppose that $x_{0} \in H, u_{0} \in U, \mu \notin \sigma(A)$ satisfy (4.3) and define $u(\cdot) \in$ $W^{1,2}[0, T ; U]$ by

$$
u(t)=u_{0}+\int_{0}^{t} v(s) d s, \quad v(\cdot)=[I-\mathscr{T}(T)]^{-1} \mathscr{C}(T)\left[A x_{0}+B u_{0}\right] .
$$

Then it follows from Lemma 2.5 that $y\left(\cdot ; x_{0}, u\right) \in W^{1,2}[0, T ; U]$ with

$$
y\left(0 ; x_{0}, u\right)=C(\mu I-A)^{-1}\left(\mu x_{0}-A x_{0}-B u_{0}\right)+T_{\mu} u_{0}=u_{0}
$$

and

$$
\dot{y}\left(\cdot ; x_{0}, u\right)=y\left(\cdot ; A x_{0}+B u_{0}, v\right)=\mathscr{C}(T)\left(A x_{0}+B u_{0}\right)+\mathscr{T}(T) v=v .
$$

Therefore we obtain from the definition of $u(\cdot)$ that $u(\cdot)=y\left(\cdot ; x_{0}, u\right)=\mathscr{C}(T) x_{0}$ $+\mathscr{T}(T) u$. But this implies $u(t)=u_{F}\left(t ; x_{0}\right)$ and, by $(4.2), S_{F}(t) x_{0}=x\left(t ; x_{0}, u\right)$ for $0 \leqslant t \leqslant T$. Hence statement (i) follows from Lemma 2.5 .

If $x_{0} \in \mathscr{D}\left(A_{F}\right)$ then

$$
S_{F}(\cdot) x_{0}=x\left(\cdot ; x_{0}, u_{F}\left(\cdot ; x_{0}\right)\right) \in \mathscr{C}^{1}[0, T ; H]
$$

and hence it follows from Lemma 2.5 (ii) that (4.4) and (4.5) hold for almost every $t>0$. Hence $S_{F}(t) x_{0} \in W_{F}$ for almost every $t>0$ and statement (ii) follows from the fact that, by (i), $W_{F}$ is invariant under $S_{F}(t)$.

The density of $W_{F}$ in $H$ follows immediately from statement (ii).

If $\left\{\left(u-T_{\mu} u, B u\right) \mid u \in U\right\} \subset U \times V$ is a closed subspace and $x_{0} \in \mathscr{D}\left(A_{F}\right)$ then it follows from (4.4), (4.5) with $t$ approaching zero that the pair $\left(C(\mu I-A)^{-1}\right.$ $\left.\cdot\left(\mu x_{0}-A_{F} x_{0}\right), A_{F} x_{0}-A x_{0}\right)$ lies in this subspace. But this implies $x_{0} \in W_{F}$.

If $C \in \mathscr{L}(H, Y)$ and $T_{\mu}=C(\mu I-A)^{-1} B$, then the closed loop input is always given by $u_{F}\left(t ; x_{0}\right)=C S_{F}(t) x_{0}$ and is in particular continuous for $t \geqslant 0$. Hence it follows from statement (ii) that $\mathscr{D}\left(A_{F}\right)=W_{F}$.

Unfortunately we were not able to determine the domain of $A_{F}$ in general and it does not seem likely that $\mathscr{D}\left(A_{F}\right)$ is always equal to $W_{F}$. Under the assumption that either the input operator or the output operator is bounded with respect to $H$ the previous theorems have been established in [40]. Furthermore, the operator $I-\mathscr{T}(t)$ is always invertible in these cases.

The next lemma establishes some elementary relations between the closed loop spectrum and the properties of $T_{\mu}$. In finite dimensions these relations provide the basis for the proof of the Nyquist criterion for the stability of feedback systems.

LEMMA 4.4. Let the assumptions of Theorem 4.2 be satisfied and let $\mu \notin \sigma(A)$ be given. Then the following statements hold.

(i) $\mu \in \operatorname{Po}\left(A_{F}\right)$ if and only if $\operatorname{ker}\left(I-T_{\mu}\right) \not \subset \operatorname{ker} B$. Furthermore, $A_{F} x_{0}=\mu x_{0}$ if and only if there exists $a u_{0} \in U$ such that $T_{\mu} u_{0}=u_{0}$ and $x_{0}=(\mu I-A)^{-1} B u_{0}$.

(ii) $\mu \in R \boldsymbol{\sigma}\left(A_{F}\right)$ if and only if

$$
\operatorname{ker}\left(I-T_{\mu}\right) \subset \operatorname{ker} B \text { and } \operatorname{cl}(\text { range } C) \not \subset \operatorname{cl}\left(\operatorname{range}\left(I-T_{\mu}\right)\right) \text {. }
$$

(iii) If $1 \notin \sigma\left(T_{\mu}\right)$ then $\mu \notin \sigma\left(A_{F}\right)$ and $\mathscr{D}\left(A_{F}\right)=W_{F}$.

Proof. If $0 \neq x_{0} \in \mathscr{D}\left(A_{F}\right)$ with $A_{F} x_{0}=\mu x_{0}$ then $S_{F}(t) x_{0}=e^{\mu t} x_{0} \in W_{F}$ for $t>0$ (Theorem 4.3 (ii)) and hence $x_{0} \in W_{F}$. Thus there exists a $u_{0} \in U$ such that (4.3) holds and therefore

$$
\mu x_{0}=A_{F} x_{0}=A x_{0}+B u_{0}
$$


and

$$
u_{0}-T_{\mu} u_{0}=C(\mu I-A)^{-1}\left(\mu x_{0}-A_{F} x_{0}\right)=0 .
$$

Since $\mu \notin \sigma(A)$ we conclude that $B u_{0} \neq 0$ and thus $\operatorname{ker}\left(I-T_{\mu}\right) \not \subset \operatorname{ker} B$.

Conversely suppose that $B u_{0} \neq 0$ and $T_{\mu} u_{0}=u_{0}$ and define $x_{0}=(\mu I-A)^{-1} B u_{0}$ $\neq 0$. Then $\mu x_{0}-A x_{0}-B u_{0}=0$ and hence (4.3) holds. Therefore $x_{0} \in W_{F} \subset$ $\mathscr{D}\left(A_{F}\right)$ and $A_{F} x_{0}=A x_{0}+B u_{0}=\mu x_{0}$. This proves statement (i).

By duality, we obtain that $\operatorname{cl}\left(\operatorname{range}\left(\mu I-A_{F}\right)\right) \neq H$ if and only if $\operatorname{cl}($ range $C) \not \subset$ $\operatorname{cl}\left(\operatorname{range}\left(I-T_{\mu}\right)\right)$. This proves statement (ii).

In order to prove statement (iii) suppose that $I-T_{\mu} \in \mathscr{L}(U)$ is invertible and choose $z_{0} \in H$. Defining

$$
u_{0}=\left(I-T_{\mu}\right)^{-1} C(\mu I-A)^{-1} z_{0} \in U \quad \text { and } \quad x_{0}=(\mu I-A)^{-1}\left(z_{0}+B u_{0}\right) \in H
$$

we obtain $z_{0}=\mu x_{0}-A x_{0}-B u_{0}$ and hence (4.3) holds. This implies $x_{0} \in W_{F}$ and $z_{0}=\mu x_{0}-A_{F} x_{0}$. Therefore $\mu I-A_{F}$ is onto. By statement (i) this operator is also one-to-one and hence $\mu \notin \sigma\left(A_{F}\right)$.

Let us now consider the case that the input operator $B \in \mathscr{L}(U, V)$ is injective and strictly unbounded with respect to $H$ so that the SCS (2.1) can be rewritten as a BCS of the form (2.12). Then the statements of Theorem 4.3 can be reformulated in a more elegant way.

COROLlaRY 4.5. Let the assumptions of Theorem 4.2 be satisfied and suppose that the BCS (2.12) is related to the SCS (2.1) through (2.8), (2.10), (2.11), (2.15). Then the following statements hold.

(i) If $x_{0} \in W_{F}=\{x \in Z \mid \Gamma x=K x\}$ then $x_{0} \in \mathscr{D}\left(A_{F}\right)$ and $A_{F} x_{0}=\Delta x_{0}$. Furthermore, $S_{F}(\cdot) x_{0} \in \mathscr{C}[0, T ; Z] \cap \mathscr{C}^{1}[0, T ; H]$ satisfies the equations

$$
\left\{\begin{array}{l}
\frac{d}{d t} S_{F}(t) x_{0}=\Delta S_{F}(t) x_{0}=S_{F}(t) \Delta x_{0}, \\
\Gamma S_{F}(t) x_{0}=K S_{F}(t) x_{0}, \quad t \geqslant 0 .
\end{array}\right.
$$

(ii) There exists a constant $c>0$ such that the following inequality holds for all $x_{0} \in W_{F}$

$$
\int_{0}^{T}\left\|K S_{F}(t) x_{0}\right\|_{U}^{2} d t \leqslant c\left\|x_{0}\right\|_{H}^{2}
$$

(iii) If either $U$ is finite dimensional or $K \in \mathscr{L}(H, Y)$, then $\mathscr{D}\left(A_{F}\right)=W_{F}$.

Proof. It follows from (2.8), (2.10), (2.11) that $x_{0} \in H$ and $u_{0} \in U$ satisfy (4.3) if and only if $x_{0} \in Z$ and $\Gamma x_{0}=u_{0}=K x_{0}$. This proves the statements (i) and (iii). Furthermore, equations (4.4) and (4.5) show that

$$
K S_{F}(\cdot) x_{0}=u_{F}\left(\cdot ; x_{0}\right)=[I-\mathscr{T}(T)]^{-1} \mathscr{T}(T) x_{0} \quad \text { for } x_{0} \in W_{F} .
$$

This implies (4.7).

5. The linear quadratic optimal control problem. In this section we consider the problem of minimizing the quadratic cost functional

$$
J(u)=\int_{0}^{T}\left[\|y(t)\|_{Y}^{2}+\|u(t)\|_{U}^{2}\right] d t
$$


where $y(t)$ is the weak output (Definition 2.4) of the wellposed SCS

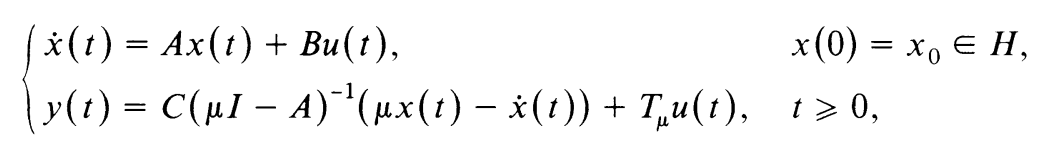

corresponding to $u(\cdot) \in L^{2}[0, T ; U]$. The optimal control will be characterized in terms of the dual SCS

$$
\begin{cases}\dot{z}(t)=-A^{*} z(t)-C^{*} y(t), & z(T)=z_{1} \in H, \\ u(t)=-B^{*}\left(\mu I-A^{*}\right)^{-1}(\mu z(t)+\dot{z}(t))-T_{\mu}^{*} y(t), & t \leqslant T,\end{cases}
$$

which is again to be understood it its weak form (Definition 3.1). Making use of the operators $\mathscr{B}(T), \mathscr{C}(T), \mathscr{T}(t)$ introduced in $\$ 2.1$ we can rewrite the coupled system (5.2), (5.3) in the equivalent form

$$
\left\{\begin{array}{l}
u=-\mathscr{B} *(T) z_{1}-\mathscr{T} *(T) y \\
y=\mathscr{C}(T) x_{0}+\mathscr{T}(T) u
\end{array}\right.
$$

For all $x_{0} \in H, z_{1} \in H$ these equations have a unique solution pair

$$
u(\cdot)=u\left(\cdot ; T, x_{0}, z_{1}\right) \in L^{2}[0, T ; U], \quad y(\cdot)=y\left(\cdot ; T, x_{0}, z_{1}\right) \in L^{2}[0, T ; Y]
$$

given by

$$
\left\{\begin{array}{l}
u\left(\cdot ; T, x_{0}, z_{1}\right)=-\left[I+\mathscr{T}^{*}(T) \mathscr{T}(T)\right]^{-1}\left(\mathscr{T}^{*}(T) \mathscr{C}(T) x_{0}+\mathscr{B} *(T) z_{1}\right), \\
y\left(\cdot ; T, x_{0}, z_{1}\right)=\left[I+\mathscr{T}(T) \mathscr{T}^{*}(T)\right]^{-1}\left(\mathscr{C}(T) x_{0}-\mathscr{T}(T) \mathscr{B} *(T) z_{1}\right) .
\end{array}\right.
$$

The corresponding solutions $x\left(t ; T, x_{0}, z_{1}\right) \in H$ of (5.2) and $z\left(t ; T, x_{0}, z_{1}\right) \in H$ of (5.3) depend continuously on all four variables and are, of course, linear in $\left(x_{0}, z_{1}\right) \in H \times H$.

For our first result on the linear quadratic control problem we need the operator $P(t) \in \mathscr{L}(H)$ defined by

$$
P(t)=\mathscr{C}^{*}(T-t)\left[I+\mathscr{T}(T-t) \mathscr{T}^{*}(T-t)\right]^{-1} \mathscr{C}(T-t)
$$

for $0 \leqslant t \leqslant T$.

THEOREM 5.1. Suppose that the SCS (5.2) is wellposed in the sense of Definition 2.4. Then for every $x_{0} \in H$ there exists a unique optimal control $u\left(\cdot ; x_{0}\right) \in L^{2}[0, T ; U]$ which minimizes the cost functional (5.1) subject to (5.2). This optimal control is characterized by (5.4) with $z_{1}=0$. The optimal output of $(5.2)$ is denoted by $y\left(t ; x_{0}\right)$ and the corresponding weak solutions $x\left(t ; x_{0}\right)$ and $z\left(t ; x_{0}\right)$ of (5.2) and (5.3), respectively, satisfy

$$
z\left(t ; x_{0}\right)=P(t) x\left(t ; x_{0}\right)
$$

for $0 \leqslant t \leqslant T$. The optimal cost is given by

$$
J(u)=\left\langle x_{0}, P(0) x_{0}\right\rangle_{H}=\left\langle\mathscr{C}(T) x_{0}, y\left(\cdot ; x_{0}\right)\right\rangle_{L^{2}[0, T ; Y]} .
$$


Proof. The cost functional $J: L^{2}[0, T ; U] \rightarrow R$ can be written in the form

$$
\begin{aligned}
J(u)= & \left\|\mathscr{C}(T) x_{0}+\mathscr{T}(T) u\right\|_{L^{2}[0, T ; Y]}^{2}+\|u\|_{L^{2}[0, T ; U]}^{2} \\
= & \left\langle x_{0}, \mathscr{C}^{*}(T) \mathscr{C}(T) x_{0}\right\rangle+2\left\langle u, \mathscr{T}^{*}(T) \mathscr{C}(T) x_{0}\right\rangle \\
& +\left\langle u, \mathscr{T}^{*}(T) \mathscr{T}(T) u+u\right\rangle .
\end{aligned}
$$

The existence and uniqueness of the optimal control follows from the fact that this quadratic functional is nondegenerate. Since its derivative vanishes at the optimal control $u(\cdot)=u\left(\cdot ; x_{0}\right) \in L^{2}[0, T ; U]$ we get

$$
u=-\mathscr{T}^{*}(T)\left(\mathscr{C}(T) x_{0}+\mathscr{T}(T) u\right)
$$

and hence $u$ is characterized by (5.4) with $z_{1}=0$. This implies

$$
y(\cdot)=y\left(\cdot ; x_{0}\right)=\left[I+\mathscr{T}(T) \mathscr{T}^{*}(T)\right]^{-1} \mathscr{C}(T) x_{0}
$$

and hence

$$
z(0)=\mathscr{C}^{*}(T)\left[I+\mathscr{T}(T) \mathscr{T}^{*}(T)\right]^{-1} \mathscr{C}(T) x_{0}=P(0) x_{0}
$$

(Corollary 3.4). In general, equation (5.6) follows from the fact that $T>0$ can be chosen arbitrarily together with the uniqueness of the optimal control. Finally, we get

$$
\begin{aligned}
J(u) & =\left\langle y, \mathscr{C}(T) x_{0}+\mathscr{T}(T) u\right\rangle+\langle u, u\rangle=\left\langle y, \mathscr{C}(T) x_{0}\right\rangle+\left\langle u+\mathscr{T}^{*}(T) y, u\right\rangle \\
& =\left\langle\left[I+\mathscr{T}(T) \mathscr{T}^{*}(T)\right]^{-1} \mathscr{C}(T) x_{0}, \mathscr{C}(T) x_{0}\right\rangle=\left\langle x_{0}, P(0) x_{0}\right\rangle .
\end{aligned}
$$

This proves the theorem.

The aim of this section is to represent the optimal control in feedback form and to derive a Riccati-type equation for the optimal cost operator $P(t)$. The main difficulty in this direction is to give a meaining to the operator $B^{*} P(t)$ since $B^{*}$ is unbounded and $P(t)$ will, in general, have no smoothing properties. This may lead to an unbounded feedback operator as was first observed by Lasiecka and Triggiani [27] in the context of the higher dimensional wave equation with Dirichlet boundary control. Another problem arises from the fact that the operator $T_{\mu}^{*}$ is needed for the representation of the output of the dual system. We will overcome these difficulties by means of studying the differentiable solutions of the coupled system (5.2), (5.3).

For notational purposes we first introduce the spaces $\mathscr{H}=H \times H, \mathscr{V}=V \times W^{*}$, $\mathscr{U}=U \times Y$ and the operators $\mathscr{A} \in \mathscr{L}(\mathscr{H}, \mathscr{V}), \mathscr{B} \in \mathscr{L}(\mathscr{U}, \mathscr{V})$ by

$$
\mathscr{A}=\left(\begin{array}{cc}
A & 0 \\
0 & -A^{*}
\end{array}\right), \quad \mathscr{B}=\left(\begin{array}{cc}
B & 0 \\
0 & -C^{*}
\end{array}\right) .
$$

Furthermore, we define $\mathscr{W} \subset \mathscr{H}$ to be the linear subspace of all pairs $\left(x_{0}, z_{0}\right) \in \mathscr{H}$ for which there exists a pair $\left(u_{0}, y_{0}\right) \in \mathscr{U}$ such that $A x_{0}+B u_{0} \in H, A^{*} z_{0}+C^{*} y_{0}$ $\in H$ and the following equations hold for some $\mu \notin \sigma(A)$ :

$$
\left\{\begin{array}{l}
u_{0}=-B^{*}\left(\mu I-A^{*}\right)^{-1}\left(\mu z_{0}-A^{*} z_{0}-C^{*} y_{0}\right)-T_{\mu}^{*} y_{0}, \\
y_{0}=C(\mu I-A)^{-1}\left(\mu x_{0}-A x_{0}-B u_{0}\right)+T_{\mu} u_{0} .
\end{array}\right.
$$


LeMma 5.2. (i) If (5.8) holds for some $\mu \notin \sigma(A)$ then it holds for every $\mu \notin \sigma(A)$.

(ii) Given $\left(x_{0}, z_{0}\right) \in \mathscr{W}$, the pair $\left(u_{0}, y_{0}\right) \in \mathscr{U}$ is uniquely determined by (5.8) and will be denoted by $\left(u_{0}, y_{0}\right)=\mathscr{F}\left(x_{0}, z_{0}\right)$.

(iii) The norm

$$
\left\|\left(x_{0}, z_{0}\right)\right\|_{\mathscr{W}}^{2}=\left\|x_{0}\right\|_{H}^{2}+\left\|A x_{0}+B u_{0}\right\|_{H}^{2}+\left\|z_{0}\right\|_{H}^{2}+\left\|A^{*} z_{0}+C^{*} y_{0}\right\|_{H}^{2}
$$

for $\left(x_{0}, z_{0}\right) \in \mathscr{W},\left(u_{0}, y_{0}\right)=\mathscr{F}\left(x_{0}, z_{0}\right)$ makes $\mathscr{W}$ into a Hilbert space with a continuous dense injection into $\mathscr{H}$.

(iv) $\mathscr{F} \in \mathscr{L}(\mathscr{W}, \mathscr{U})$ and $\mathscr{A}+\mathscr{B} \mathscr{F} \in \mathscr{L}(\mathscr{W}, \mathscr{H})$. Moreover, $\mathscr{A}+\mathscr{B} \mathscr{F}$ is a closed operator on $\mathscr{H}$.

Proof. Statement (i) follows straightforwardly from the compatibility condition (2.3). In order to establish statement (ii) suppose that (5.8) holds with $x_{0}=z_{0}=0$. Then $B u_{0} \in H, C^{*} y_{0} \in H$ and

$$
\left\langle y_{0}, y_{0}\right\rangle=-\left\langle y_{0}, C(\mu I-A)^{-1} B u_{0}\right\rangle+\left\langle y_{0}, T_{\mu} u_{0}\right\rangle=-\left\langle u_{0}, u_{0}\right\rangle
$$

which implies $u_{0}=0, y_{0}=0$. Now observe that the unique solution $\left(u_{0}, y_{0}\right) \in \mathscr{U}$ of (5.8) depends continuously on $x_{0} \in H, A x_{0}+B u_{0} \in H, z_{0} \in H, A^{*} z_{0}+C^{*} y_{0} \in$ $H$. This implies that the norm in (iii) makes $\mathscr{W}$ into a Hilbert space and (equivalently) that $\mathscr{A}+\mathscr{B} \mathscr{F}: \mathscr{W} \rightarrow \mathscr{H}$ is a closed operator on $\mathscr{H}$. Note that the norm on $\mathscr{W}$ is precisely the graph norm of $\mathscr{A}+\mathscr{B} \mathscr{F}$. Furthermore, we obtain $\mathscr{F} \in \mathscr{L}(\mathscr{W}, \mathscr{U})$.

It remains to show that $\mathscr{W}$ is dense in $\mathscr{H}$. For this purpose let $x_{0} \in H$, $u(\cdot) \in L^{2}[0, T ; U]$ be given and let $x(\cdot) \in \mathscr{C}[0, T ; H] \cap W^{1,2}[0, T ; V]$ and $y(\cdot) \in$ $L^{2}[0, T ; Y]$ be the unique solution and output, respectively, of (5.2) in the weak sense. Then

$$
\bar{x}=\int_{0}^{T} x(t) d t, \quad \bar{u}=\int_{0}^{T} u(t) d t, \quad \bar{y}=\int_{0}^{T} y(t) d t
$$

satisfy

$$
A \bar{x}+B \bar{u}=x(T)-x_{0} \in H
$$

and

$$
\bar{y}=C(\mu I-A)^{-1}(\mu \bar{x}-A \bar{x}-B \bar{u})+T_{\mu} \bar{u} .
$$

The latter equation has first to be established for $u(\cdot) \in W^{1,2}[0, T ; U]$ with $A x_{0}+$ $B u(0) \in H$ and follows in general from continuous dependence. Since the same arguments apply to system (5.3) we get

$$
\frac{1}{T}\left(\int_{0}^{T} x\left(t ; T, x_{0}, z_{1}\right) d t, \int_{0}^{T} z\left(t ; T, x_{0}, z_{1}\right) d t\right) \in \mathscr{W}
$$

for all $\left(x_{0}, z_{1}\right) \in \mathscr{H}$ and all $T>0$. Therefore $\mathscr{W}$ is dense in $\mathscr{H}$.

The next and most important step in the development of this section is the characterization of those pairs $x_{0}, z_{1}$ for which the corresponding solutions of (5.4) are differentiable. For this purpose we introduce the subspace $\mathscr{W}(T) \subset \mathscr{H}$ of all those pairs $\left(x_{0}, z_{1}\right) \in \mathscr{W}$ for which there exists a pair $\left(u_{0}, y_{1}\right) \in \mathscr{U}$ such that 
$A x_{0}+B u_{0} \in H, A^{*} z_{1}+C^{*} y_{1} \in H$ and the following equations hold for some $\mu \notin \sigma(A)$ :

$$
\begin{gathered}
u_{0}+\int_{0}^{T} u\left(t ; T, A x_{0}+B u_{0},-A^{*} z_{1}-C^{*} y_{1}\right) d t \\
=-B^{*}\left(\mu I-A^{*}\right)^{-1}\left(\mu z_{1}-A^{*} z_{1}-C^{*} y_{1}\right)-T_{\mu}^{*} y_{1}, \\
y_{1}-\int_{0}^{T} y\left(t ; T, A x_{0}+B u_{0},-A^{*} z_{1}-C^{*} y_{1}\right) d t \\
=C(\mu I-A)^{-1}\left(\mu x_{0}-A x_{0}-B u_{0}\right)+T_{\mu} u_{0} .
\end{gathered}
$$

REMARKS 5.3. (i) Note that equations (5.9) are independent of $\mu \notin \sigma(A)$. Furthermore, the next lemma shows that $u_{0} \in U$ and $y_{1} \in Y$ are uniquely determined by (5.9) if $\left(x_{0}, z_{1}\right) \in \mathscr{W}(T)$ is given. Finally, $u_{0}$ and $y_{1}$ depend continuously on $x_{0} \in H, \quad A x_{0}+B u_{0} \in H, \quad z_{1} \in H, \quad A^{*} z_{1}+C^{*} y_{1} \in H$. This allows us to make $\mathscr{W}(T)$ into a Hilbert space by defining

$$
\left\|\left(x_{0}, z_{1}\right)\right\|_{\mathscr{W}(T)}^{2}=\left\|x_{0}\right\|_{H}^{2}+\left\|A x_{0}+B u_{0}\right\|_{H}^{2}+\left\|z_{1}\right\|_{H}^{2}+\left\|A^{*} z_{1}+C^{*} y_{1}\right\|_{H}^{2}
$$

for $\left(x_{0}, z_{1}\right) \in \mathscr{W}(T)$ where $u_{0} \in U$ and $y_{1} \in Y$ are chosen such that (5.9) holds.

(ii) The operator $\mathscr{F}(T): \mathscr{W}(T) \rightarrow \mathscr{U}$ defined by $\mathscr{F}(T)\left(x_{0}, z_{1}\right)=\left(u_{0}, y_{1}\right)$ for $\left(x_{0}, z_{1}\right) \in \mathscr{W}(T),\left(u_{0}, y_{1}\right) \in \mathscr{U}$ satisfying (5.9) is bounded and linear.

(iii) $\mathscr{A}+\mathscr{B} \mathscr{F}(T)$ is a bounded operator from $\mathscr{W}(T)$ into $\mathscr{H}$ and a closed operator on $\mathscr{H}$. The norm on $\mathscr{W}(T)$ is precisely the graph norm of $\mathscr{A}+\mathscr{B} \mathscr{F}(T)$. Unfortunately we were not able to show that $\mathscr{W}(T)$ is dense in $\mathscr{H}$. We leave this as a conjecture.

(iv) Note that $\mathscr{W}(0)=\mathscr{W}$ and $\mathscr{F}(0)=\mathscr{F}$.

Finally, we introduce the operator $\mathscr{P}(t ; T) \in \mathscr{L}(\mathscr{H})$ by defining

$$
\mathscr{S}(t ; T)=\left(\begin{array}{cc}
I & Q(t) \\
-P(t) & I
\end{array}\right)^{-1}\left(\begin{array}{cc}
S(t)-R(t) & 0 \\
0 & S *(T-t)-R^{*}(T-t)
\end{array}\right)
$$

$$
\begin{gathered}
Q(t)=\mathscr{B}(t)\left[I+\mathscr{T}^{*}(t) \mathscr{T}(t)\right]^{-1} \mathscr{B} *(t), \\
R(t)=\mathscr{B}(t)\left[I+\mathscr{T}^{*}(t) \mathscr{T}(t)\right]^{-1} \mathscr{T}^{*}(t) \mathscr{C}(t),
\end{gathered}
$$

for $0 \leqslant t \leqslant T$.

Lemma 5.4. Suppose that the SCS (5.2) is wellposed in the sense of Definition 2.4, let $\left(x_{0}, z_{1}\right) \in \mathscr{H}$ be given and let $u(t)=u\left(t ; T, x_{0}, z_{1}\right), y(t)=y\left(t ; T, x_{0}, z_{1}\right), x(t)=$ $x\left(t ; T, x_{0}, z_{1}\right), z(t)=z\left(t ; T, x_{0}, z_{1}\right)$ be defined as above. Then the following statements hold.

(i) For every $t \in[0, T]$

$$
(x(t), z(t))=\mathscr{S}(t ; T)\left(x_{0}, z_{1}\right) .
$$

(ii) $\left(x_{0}, z_{1}\right) \in \mathscr{W}(T)$ if and only if $x(\cdot) \in \mathscr{C}^{1}[0, T ; H], z(\cdot) \in \mathscr{C}^{1}[0, T ; H], u(\cdot) \in$ $W^{1,2}[0, T ; U], y(\cdot) \in W^{1,2}[0, T ; Y]$. 
(iii) If $\left(x_{0}, z_{1}\right) \in \mathscr{W}(T)$ then $\dot{x}(t), \dot{z}(t), \dot{u}(t), \dot{y}(t)$ are the weak solutions of (5.2) and (5.3) with $\left(x_{0}, z_{1}\right)$ replaced by $(\mathscr{A}+\mathscr{B} \mathscr{F}(T))\left(x_{0}, z_{1}\right)$. Moreover, $(x(s), z(t)) \in$ $\mathscr{W}(t-s)$ for $0 \leqslant s \leqslant t \leqslant T$ and

$$
(u(s), y(t))=\mathscr{F}(t-s)(x(s), z(t)) .
$$

(iv) If $\left(x_{0}, z_{1}\right) \in \mathscr{W}(T)$, then $\mathscr{S}(\cdot ; T)\left(x_{0}, z_{1}\right) \in \mathscr{C}[0, T ; \mathscr{W}] \cap \mathscr{C}^{1}[0, T ; \mathscr{H}]$ and

$$
\begin{aligned}
\frac{d}{d t} \mathscr{S}(t ; T)\left(x_{0}, z_{1}\right) & =[\mathscr{A}+\mathscr{B} \mathscr{F}] \mathscr{S}(t ; T)\left(x_{0}, z_{1}\right) \\
& =\mathscr{S}(t ; T)[\mathscr{A}+\mathscr{B} \mathscr{F}(T)]\left(x_{0}, z_{1}\right)
\end{aligned}
$$

for $0 \leqslant t \leqslant T$.

Proof. It follows from (5.5) that

$$
\begin{gathered}
x(t)=S(t) x_{0}-\mathscr{B}(t)\left[I+\mathscr{T}^{*}(t) \mathscr{T}(t)\right]^{-1}\left(\mathscr{T}^{*}(t) \mathscr{C}(t) x_{0}+\mathscr{B} *(t) z(t)\right), \\
z(t)=S^{*}(T-t) z_{1}+\mathscr{C}^{*}(T-t)\left[I+\mathscr{T}(T-t) \mathscr{T}^{*}(T-t)\right]^{-1} \\
\cdot\left(\mathscr{C}(T-t) x(t)-\mathscr{T}(T-t) \mathscr{B} *(T-t) z_{1}\right),
\end{gathered}
$$

for $0 \leqslant t \leqslant T$. This proves (5.11). In order to prove statement (ii) let us first assume that $x(\cdot) \in \mathscr{C}^{1}[0, T ; H], \quad z(\cdot) \in \mathscr{C}^{1}[0, T ; H], \quad u(\cdot) \in W^{1,2}[0, T ; U], \quad y(\cdot) \in$ $W^{1,2}[0, T ; Y]$. Then it follows from Lemma 2.5 and Lemma 3.2 that (5.9) holds with $u_{0}=u(0), \quad y_{1}=y(T)$. Conversely, assume that $\left(x_{0}, z_{1}\right) \in \mathscr{W}(T), \quad\left(u_{0}, y_{1}\right)=$ $\mathscr{F}(T)\left(x_{0}, z_{1}\right)$ and define

$$
\begin{aligned}
& u(t)=u_{0}+\int_{0}^{t} u\left(s ; T, A x_{0}+B u_{0},-A^{*} z_{1}-C^{*} y_{1}\right) d s, \\
& y(t)=y_{1}-\int_{t}^{T} y\left(s ; T, A x_{0}+B u_{0},-A^{*} z_{1}-C^{*} y_{1}\right) d s
\end{aligned}
$$

for $0 \leqslant t \leqslant T$. Then Lemma 2.5 shows that $y\left(\cdot ; x_{0}, u\right) \in W^{1,2}[0, T ; Y]$ satisfies

$$
\dot{y}\left(\cdot ; x_{0}, u\right)=y\left(\cdot ; A x_{0}+B u_{0}, \dot{u}\right)=y\left(\cdot ; A x_{0}+B u_{0},-A^{*} z_{1}-C^{*} y_{1}\right)=\dot{y}(\cdot) \text {. }
$$

Furthermore, it follows from $(5.9 ; 2)$ that $y(0)=y\left(0 ; x_{0}, u\right)$ and hence $y(\cdot)=$ $y\left(\cdot ; x_{0}, u\right)$. Analogous arguments using Lemma 3.2 and $(5.9 ; 1)$ show that $u(\cdot)=$ $-w\left(\cdot ; z_{1}, y\right)$. Hence $u(\cdot)$ and $y(\cdot)$ satisfy (5.4) and we conclude that $u(\cdot)=$ $u\left(\cdot ; T, x_{0}, z_{1}\right)$ and $y(\cdot)=y\left(\cdot ; T, x_{0}, z_{1}\right)$. This proves the statements (ii) and (iii). Finally, statement (iv) is an operator theoretic reformulation of statement (iii).

In order to apply the previous lemma to the linear quadratic control problem we introduce the Hilbert space $W(t)=\left\{x_{0} \in H \mid\left(x_{0}, 0\right) \in \mathscr{W}(T-t)\right\}$ with the norm

$$
\left\|x_{0}\right\|_{W(t)}^{2}=\left\|x_{0}\right\|_{H}^{2}+\left\|A x_{0}+B u_{0}\right\|_{H}^{2}+\left\|C^{*} y_{1}\right\|_{H}^{2} \text { for } x_{0} \in W(t)
$$

where $u_{0}=: F(t) x_{0} \in U$ and $y_{1} \in Y$ are chosen such that (5.9) holds with $z_{1}=0$ and $T$ replaced by $T-t$ (see Remark 5.3(i)). Then $F(t) \in \mathscr{L}(W(t), U), A+B F(t)$ $\in \mathscr{L}(W(t), H)$ and we define $K(t) \in \mathscr{L}(W(t), Y)$ by

$$
K(t)=C(\mu I-A)^{-1}(\mu I-A-B F(t))+T_{\mu} F(t)
$$

for $0 \leqslant t \leqslant T$. Finally, we define $\Phi(t, s)$ for $0 \leqslant s \leqslant t \leqslant T$ by

$$
\Phi(t, s)=\left[\begin{array}{ll}
I & 0
\end{array}\right] \mathscr{S}(t-s ; T-s)\left[\begin{array}{l}
I \\
0
\end{array}\right] .
$$


THEOREM 5.5. Suppose that the SCS (5.2) is wellposed in the sense of Definition 2.4 and let $x_{0} \in H$ be given. Then the following statements hold.

(i) $x_{0} \in W(0)$ if and only if $u\left(\cdot ; x_{0}\right) \in W^{1,2}[0, T ; U], y\left(\cdot ; x_{0}\right) \in W^{1,2}[0, T ; Y]$, $x\left(\cdot ; x_{0}\right) \in \mathscr{C}^{1}[0, T ; H], z\left(\cdot ; x_{0}\right) \in \mathscr{C}^{1}[0, T ; H]$. If $x_{0} \in W(0)$ then $x(t) \in W(t)$ for $0 \leqslant t \leqslant T$ and

$$
u\left(t ; x_{0}\right)=F(t) x\left(t ; x_{0}\right), \quad y\left(t ; x_{0}\right)=K(t) x\left(t ; x_{0}\right) .
$$

(ii) The operator $\Phi(t, s) \in \mathscr{L}(H) \cap \mathscr{L}(W(s), W(t))$ satisfies $\Phi(t, \tau) \Phi(\tau, s)=$ $\Phi(t, s), \Phi(s, s)=I$ and

$$
x\left(t ; x_{0}\right)=\Phi(t, s) x\left(s ; x_{0}\right)
$$

for $0 \leqslant s \leqslant \tau \leqslant t \leqslant T$. If $x \in W(s)$ then $\Phi(\cdot, s) x \in \mathscr{C}^{1}[s, T ; H]$ and

$$
\frac{d}{d t} \Phi(t, s) x=[A+B F(t)] \Phi(t, s) x=\Phi(t, s)[A+B F(s)] x \text {. }
$$

(iii) If $x \in W(t)$ then $(x, P(t) x) \in \mathscr{W}$ and

$$
(F(t) x, K(t) x)=\mathscr{F}(x, P(t) x)
$$

for $0 \leqslant t \leqslant T$. Furthermore the following equation holds for $0 \leqslant t \leqslant T$ and $x, z \in$ $W(t)$

$$
\begin{aligned}
& \langle z, P(t) x\rangle=\int_{t}^{T}\langle\mathscr{F}(\Phi(\tau, t) z, P(\tau) \Phi(\tau, t) z) \\
& \mathscr{F}(\Phi(\tau, t) x, P(\tau) \Phi(\tau, t) x)\rangle_{U \times Y} d \tau
\end{aligned}
$$

Proof. Theorem 5.1 and Lemma 5.4.

An essential feature of the above theorem is that the feedback operator $F(t) \in$ $\mathscr{L}(W(t), U)$ is unbounded with respect to $H$ and will in general have no bounded extension. Furthermore, it is important to note that $F(t) x \in U$ depends not only on $P(t) x$ but also on $x$ itself (see equation (5.19)). This change to the product space $\mathscr{W} \subset H \times H$ is the key feature in equation (5.20) which may be considered as a generalized version of the integral Riccati equation (compare Curtain and Pritchard [6], Gibson [17], Pritchard and Salamon [34]). If the input and output operators $B$ and $C$ are bounded with respect to $H$ then $F(t)$ is bounded as well and furthermore $W(t)=W$ in that case. The latter follows from equation (5.5) in connection with the fact that the operator $I+\mathscr{T}^{*}(T) \mathscr{T}(T)$ is boundedly invertible on $W^{1,2}[0, T ; U]$ if $B$ and $C$ are bounded. Unfortunately we were not able to prove in general that $W(t)$ is dense in $H$, and we leave this as a conjecture. Another interesting open question is whether there is a way to differentiate equation (5.20) in order to derive some kind of a differential Riccati equation for the operator $P(t)$. A question which we have not addressed is the uniqueness for the solution operator $P(t)$ of (5.20). Finally we point out that a cost functional with an (arbitrary nonnegative) additional weighting term on the final state $x(T)$ can be treated in an analogous way as presented in this section.

We close this section with the discussion of four interesting special cases.

COROllary 5.6 (BOUNDED INPUT OPERATOR). Suppose that the SCS (5.2) is wellposed in the sense of Definition 2.4, that $B \in \mathscr{L}(U, H)$ and that $T_{\mu}=$ $C(\mu I-A)^{-1} B$. Then the following statements hold. 
(i) $\mathscr{W}=\left\{\left(x_{0}, z_{0}\right) \in W \times H \mid A^{*} z_{0}+C^{*} C x_{0} \in H\right\}$ and $\mathscr{F}\left(x_{0}, z_{0}\right)=$ $\left(-B^{*} z_{0}, C x_{0}\right)$ for $\left(x_{0}, z_{0}\right) \in \mathscr{W}$. The space $W(t)$ consists of all $x_{0} \in W$ for which there exists $a y_{1} \in Y$ such that $C^{*} y_{1} \in H$ and

$$
\begin{aligned}
& B^{*} P(t) x_{0}=\int_{0}^{T-t} u\left(s ; T-t, A x_{0}-B B^{*} P(t) x_{0},-C^{*} y_{1}\right) d s, \\
& y_{1}=C x_{0}+\int_{0}^{T-t} y\left(s ; T-t, A x_{0}-B B^{*} P(t) x_{0},-C^{*} y_{1}\right) d s .
\end{aligned}
$$

Moreover $F(t) x=-B^{*} P(t) x$ and $K(t) x=C x$ for $x \in W(t)$.

(ii) The optimal control is always continuous and characterized by the bounded feedback law

$$
u\left(t ; x_{0}\right)=-B^{*} P(t) x\left(t ; x_{0}\right) .
$$

Moreover, the following equation holds for all $x \in H$ and $0 \leqslant s \leqslant t \leqslant T$ :

$$
\begin{aligned}
\Phi(t, s) x & =S(t-s) x-\int_{s}^{t} S(t-\tau) B B^{*} P(\tau) \Phi(\tau, s) x d \tau \\
& =S(t-s) x-\int_{s}^{t} \Phi(t, \tau) B B^{*} P(\tau) S(\tau-s) x d \tau .
\end{aligned}
$$

(iii) The following equation holds for all $t \in[0, T]$ and all $x, z \in W$

$$
\begin{aligned}
\langle z, P(t) x\rangle= & \int_{t}^{T}\langle C S(\tau-t) z, C S(\tau-t) x\rangle d \tau \\
& -\int_{t}^{T}\left\langle B^{*} P(\tau) S(\tau-t) z, B^{*} P(\tau) S(\tau-t) x\right\rangle d \tau .
\end{aligned}
$$

(iv) If $x \in W$ then $P(\cdot) x \in \mathscr{C}^{1}\left[0, T ; W^{*}\right]$ and the following equation holds for $0 \leqslant t \leqslant T$

$$
\frac{d}{d t} P(t) x+A^{*} P(t) x+P(t) A x-P(t) B B^{*} P(t) x+C^{*} C x=0 .
$$

Proof. It follows from (5.4) and (5.6) that

$$
u\left(t ; x_{0}\right)=-B^{*} z\left(t ; x_{0}\right)=-B^{*} P(t) x\left(t ; x_{0}\right)
$$

for all $t \in[0, T]$ and all $x_{0} \in H$. This proves statement (ii). Statement (i) follows from statement (ii) and equation (5.9).

In order to prove statement (iii) we make use of the fact that the operator $\mu(\mu I-A)^{-1}$ converges strongly to the identity in $\mathscr{L}(H)$ as $\mu$ approaches $+\infty$. We replace $C \in \mathscr{L}(W, Y)$ by $C_{\mu}=\mu C(\mu I-A)^{-1} \in \mathscr{L}(H, Y)$ and denote by $\mathscr{C}_{\mu}(T)$, $\mathscr{T}_{\mu}(T), P_{\mu}(t), 0 \leqslant t \leqslant T$, the operators which replace $\mathscr{C}(T), \mathscr{T}(T), P(t)$, respectively. Using hypothesis (S3) in $\S 2.1$ one shows easily that the operators $\mathscr{C}_{\mu}(T)$, $\mathscr{C}_{\mu}{ }^{*}(T), \mathscr{T}_{\mu}(T), \mathscr{T}_{\mu}^{*}(T)$ converge to $\mathscr{C}(T), \mathscr{C}^{*}(T), \mathscr{T}(T), \mathscr{T}^{*}(T)$, respectively, in the strong operator topology. In the case of the operator $\mathscr{T}(T)$ it is useful to consider first the case $B=I, U=H$ and to recall that the SCS (5.2) is wellposed in this case by duality. Hence the operator $P_{\mu}(t)$ converges to $P(t)$ in the strong operator topology. It is well known $[6,17,34]$ that the operators $P_{\mu}(t)$ satisfy equation (5.24) with $C$ replaced by $C_{\mu}$. Since $\left\|P_{\mu}(t)\right\| \leqslant\left\|P_{\mu}(0)\right\|$ we can apply the dominated convergence theorem to these equations and get (5.24). 
Now statement (iv) can be established by differentiating (5.24) as in [34]. In a straightforward way we obtain the following weak form of (5.25) for $x, z \in \mathscr{D}\left(A^{2}\right)$.

$$
\begin{aligned}
\frac{d}{d t}\langle z, P(t) x\rangle+\langle A x, P(t)\rangle+\langle P(t) z, A x\rangle & \\
& -\left\langle B^{*} P(t) z, B^{*} P(t) x\right\rangle+\langle C z, C x\rangle=0 .
\end{aligned}
$$

By continuous extension this equation holds for all $x, z \in W$. Furthermore, the equation can be rewritten in the form

$$
\langle z, P(t) x\rangle=\left\langle z, \int_{t}^{T}\left[A^{*} P(s) x+P(s) A x-P(s) B B^{*} P(s) x+C^{*} C x\right] d s\right\rangle_{W, W^{*}} .
$$

This proves (5.25).

The differential Riccati equation (5.25) in Corollary 5.4 has been established in [34] under the additional assumption that $B \in \mathscr{L}(U, H)$ satisfies hypothesis (H2) with $H$ replaced by $W$. Under this condition also the uniqueness for nonnegative solutions of (5.25) has been shown in [34] and one can easily see that $\Phi(t, s) \in \mathscr{L}(W)$. It seems that the latter does not hold in general under the assumptions of Corollary 5.4

COROllary 5.7 (BOUNDED OUTPUT OPERATOR). Suppose that the SCS (5.2) is wellposed in the sense of Definition 2.4 and that $C \in \mathscr{L}(H, Y)$ and $T_{\mu}=$ $C(\mu I-A)^{-1} B$. Then the following statements hold.

(i) $\mathscr{W}=\left\{\left(x_{0}, z_{0}\right) \in H \times V^{*} \mid A x_{0}-B B^{*} z_{0} \in H\right\}$ and $\mathscr{F}\left(x_{0}, z_{0}\right)=$ $\left(-B^{*} z_{0}, C x_{0}\right)$ for $\left(x_{0}, z_{0}\right) \in \mathscr{W}$. The space $W(t)$ consists of all $x_{0} \in H$ for which there exist $u_{0} \in U, y_{1} \in Y$ such that $A x_{0}+B u_{0} \in H$ and

$$
\begin{aligned}
& u_{0}+\int_{0}^{T-t} u\left(s ; T-t, A x_{0}+B u_{0},-C^{*} y_{1}\right) d s=0, \\
& y_{1}=C x_{0}+\int_{0}^{T-t} y\left(s ; T-t, A x_{0}+B u_{0},-C^{*} y_{1}\right) d s .
\end{aligned}
$$

If these equations hold, then $P(t) x_{0} \in V^{*}$ and $F(t) x_{0}=-B^{*} P(t) x_{0}=u_{0}$ and $K(t) x_{0}=C x_{0}$.

(ii) The following equation holds for all $x, z \in H$ and $0 \leqslant t \leqslant T$,

$$
\langle z, P(t) x\rangle=\int_{t}^{T}\langle C \Phi(\tau, t) z, C S(\tau-t) x\rangle d \tau .
$$

(iii) The following equation holds for $0 \leqslant t \leqslant T$ and $x, z \in W(t)$ :

$$
\begin{aligned}
\langle z, P(t) x\rangle= & \int_{t}^{T}\langle C \Phi(\tau, t) z, C \Phi(\tau, t) x\rangle d \tau \\
& +\int_{t}^{T}\left\langle B^{*} P(\tau) \Phi(\tau, t) z, B^{*} P(\tau) \Phi(\tau, t) x\right\rangle d \tau .
\end{aligned}
$$

Proof. It follows from Theorem 5.3 that $\left\langle x_{0}, P(0) x_{0}\right\rangle=\left\langle y\left(\cdot ; x_{0}\right), \mathscr{C}(T) x_{0}\right\rangle$. This proves (5.27) for $t=0$ and $x=z$. In general, (5.27) follows from the fact that $P(t)$ is selfadjoint and plays the role of $P(0)$ for the control problem on the time interval $[t, T]$. Statements (i) and (iii) follow from (5.9) and Theorem 5.5. 
COROllary 5.8 (STRICTLY UNBOUNDED INPUT OPERATOR). Suppose that the SCS (5.2) is wellposed in the sense of Definition 2.4, that $B$ is injective and strictly unbounded with respect to $H$ and that the operators $\Delta, \Gamma, K$ are related to the SCS (5.2) as in \$2.2. Then the following statements hold.

(i) $\mathscr{W}=\left\{\left(x_{0}, z_{0}\right) \in Z \times H \mid \Delta^{*} z_{0}+K^{*} K x_{0}+\Gamma^{*} \Gamma x_{0} \in H\right\}$ and $\mathscr{F}\left(x_{0}, z_{0}\right)=$ $\left(\Gamma x_{0}, K x_{0}\right)$ for $\left(x_{0}, z_{0}\right) \in \mathscr{W}$. The space $W(t)$ consists of all $x_{0} \in Z$ for which there exist $u_{1} \in U$ and $y_{1} \in Y$ such that $K^{*} y_{1}+\Gamma^{*} u_{1} \in H$ and

$$
\left\{\begin{array}{l}
\Gamma x_{0}+\int_{0}^{T-t} u\left(s ; T-t, \Delta x_{0},-K^{*} y_{1}-\Gamma^{*} u_{1}\right) d s=-u_{1}, \\
K x_{0}+\int_{0}^{T-t} y\left(s ; T-t, \Delta x_{0},-K^{*} y_{1}-\Gamma^{*} u_{1}\right) d s=y_{1}
\end{array}\right.
$$

If these equations hold then $\Delta^{*} P(t) x_{0}+K^{*} K x_{0}+\Gamma^{*} \Gamma x_{0} \in H$ and $F(t) x_{0}=\Gamma x_{0}$, $K(t) x_{0}=K x_{0}$.

(ii) For every $x_{0} \in H$ the adjoint state $z(\cdot)=z\left(\cdot ; x_{0}\right) \in \mathscr{C}[0, T ; H] \cap$ $W^{1,2}\left[0, T ; Z^{*}\right]$ and the optimal control $u(\cdot)=u\left(\cdot ; x_{0}\right) \in L^{2}[0, T ; U]$ and the optimal output $y(\cdot)=y\left(\cdot ; x_{0}\right) \in L^{2}[0, T ; Y]$ satisfy the equation

$$
\dot{z}(t)+\Gamma^{*} u(t)=-\Delta^{*} z(t)-K^{*} y(t), \quad z(T)=0 .
$$

If moreover $x_{0} \in W(0)$, then $z(\cdot) \in \mathscr{C}^{1}[0, T ; H]$ and $x(:)=x\left(\cdot ; x_{0}\right) \in \mathscr{C}[0, T ; Z] \cap$ $\mathscr{C}^{1}[0, T ; H]$ satisfies the equation

$$
\dot{x}(t)=\Delta x(t), \quad \Gamma x(t)=u(t), \quad y(t)=\dot{K} x(t), \quad x(0)=x_{0} .
$$

(iii) The following equation holds for all $t \in[0, T]$ and all $x, z \in W(t)$ :

$$
\begin{aligned}
\langle z, P(t) x\rangle= & \int_{t}^{T}\langle K \Phi(\tau, t) z, K \Phi(\tau, t) x\rangle_{Y} d \tau \\
& +\int_{t}^{T}\langle\Gamma \Phi(\tau, t) z, \Gamma \Phi(\tau, t) x\rangle_{U} d \tau .
\end{aligned}
$$

Proof. Statement (i) follows from (5.9) together with the fact that $A^{*} z_{1}+C^{*} y_{1}$ $\in H$ and $B^{*}\left(\mu I-A^{*}\right)^{-1}\left(\mu z_{1}-A^{*} z_{1}-C^{*} y_{1}\right)+T_{\mu}^{*} y_{1}=-u_{1}$ is equivalent to $\Delta^{*} z_{1}$ $+K^{*} y_{1}+\Gamma^{*} u_{1} \in H$ (Lemma 2.2). The remaining assertions of the corollary follow directly from Theorem 5.5.

COROLlARY 5.9 (STRICTLY UNBOUNDED OUTPUT OPERATOR). Suppose that the SCS (5.2) is wellposed in the sense of Definition 2.4, that $C$ has a dense range and is strictly unbounded with respect to $H$ and that the operators $\Lambda, \Omega, G$ are related to the SCS SCS (5.2) as in \$2.3. Then the following statements hold.

(i) $\mathscr{W}=\left\{\left(x_{0}, z_{0}\right) \in H \times X^{*} \mid \Lambda x_{0}-G G^{*} z_{0}-\Omega \Omega *_{z_{0}} \in H\right\}$ and $\mathscr{F}\left(x_{0}, z_{0}\right)=$ $\left(-G * z_{0}, \Omega^{*} z_{0}\right)$ for $\left(x_{0}, z_{0}\right) \in \mathscr{W}$. The space $W(t)$ consists of all $x_{0} \in H$ for which there exist $u_{0} \in U$ and $y_{0} \in Y$ such that $\Lambda x_{0}+G u_{0}-\Omega y_{0} \in H$ and

$$
\left\{\begin{array}{l}
u_{0}+\int_{0}^{T-t} u\left(s ; T-t, \Lambda x_{0}+G u_{0}-\Omega y_{0}, 0\right) d s=0 \\
y_{0}+\int_{0}^{T-t} y\left(s ; T-t, \Lambda x_{0}+G u_{0}-\Omega y_{0}, 0\right) d s=0
\end{array}\right.
$$

If these equations hold then $P(t) x_{0} \in X^{*}$ and $F(t) x_{0}=-G^{*} P(t) x_{0}=u_{0}$ and $K(t) x_{0}=\Omega * P(t) x_{0}=y_{0}$. 
(ii) For every $x_{0} \in H$ the optimal state trajectory $x(\cdot)=x\left(\cdot ; x_{0}\right) \in \mathscr{C}[0, T ; H] \cap$ $W^{1,2}[0, T ; X]$ and the optimal control $u(\cdot)=u\left(\cdot ; x_{0}\right) \in L^{2}[0, T ; U]$ and the optimal output $y(\cdot)=y\left(\cdot ; x_{0}\right) \in L^{2}[0, T ; Y]$ satisfy the equation

$$
\dot{x}(t)+\Omega y(t)=\Lambda x(t)+G u(t), \quad x(0)=x_{0} .
$$

If moreover $x_{0} \in W(0)$ then $x(\cdot) \in \mathscr{C}^{1}[0, T ; H]$ and $z(\cdot)=z\left(\cdot ; x_{0}\right) \in \mathscr{C}\left[0, T ; X^{*}\right]$ $\cap \mathscr{C}^{1}[0, T ; H]$ satisfies the equation

$$
\dot{z}(t)=-\Lambda^{*} z(t), \quad \Omega^{*} z(t)=y(t), \quad u(t)=-G^{*} z(t), \quad z(T)=0 .
$$

(iii) The following equation holds for all $t \in[0, T]$ and all $x, z \in W(t)$ :

$$
\begin{aligned}
\langle z, P(t) x\rangle= & \int_{t}^{T}\left\langle\Omega^{*} P(\tau) \Phi(\tau, t) z, \Omega^{*} P(\tau) \Phi(\tau, t) x\right\rangle_{Y} d \tau \\
& +\int_{t}^{T}\left\langle G^{*} P(\tau) \Phi(\tau, t) z, G^{*} P(\tau) \Phi(\tau, t) x\right\rangle_{U} d \tau .
\end{aligned}
$$

Proof. Lemma 2.12 and Theorem 5.5.

The linear quadratic control problem (LQCP) for infinite dimensional systems with unbounded control and observation has previously been studied e.g. by Lukes and Russell [30], Russell [37], Lions [28], Balakrishnan [2], Lasiecka and Triggiani [25, 27], Flandoli [13], Sorine [42, 43] for various classes of partial differential equations (PDE) and by Ichikawa [20], Datko [9], Delfour [10], Ito and Tarn [21], Pritchard and Salamon [34], Karrakchou [22], for retarded and neutral functional differential equations (FDE). A general semigroup theoretic framework for the LQCP which allows for unbounded input and output operators and applies to large classes of PDEs and FDEs has been presented in [34]. However, the "degree of unboundedness" in the input and output operators which can be allowed in [34] is not general enough to cover all cases of interest. In the theory developed in this section there are no requirements on the operators $A, B, C, T_{\mu}$ other than wellposedness. In this sense our approach includes all previous results on the LQCP for wellposed control systems. However, more specific conclusions and results are certainly possible under more restrictive assumptions. In the case of analytic semigroups for example we refer to Flandoli [13], Sorine [42, 43], Lasiecka and Triggiani [25], DaPrato [8] and for the LQCP on the infinite time interval under stronger hypotheses to Pritchard and Salamon [34]. Moreover, we mention the recent paper by Flandoli [14] which contains a very nice approach to the LQCP for nonwellposed Cauchy problems.

6. Functional differential equations. The aim of this section is to show how a very general class of neutral functional differential equations (NFDE) fits into the framework of $\S 2$ so that the results of $\S \S 4$ and 5 can be applied. Consider the NFDE

$$
\left\{\begin{array}{l}
\frac{d}{d t}\left(x(t)-M x_{t}-G u_{t}\right)=L x_{t}+B u_{t}, \\
y(t)=C x_{t}+D u_{t},
\end{array}\right.
$$

where $u(t) \in R^{m}, x(t) \in R^{n}, y(t) \in R^{p}$ and $x_{t}$ is defined by $x_{t}(\tau)=x(t+\tau)$ for $-h \leqslant \tau \leqslant 0$ where $0<h<\infty$. Correspondingly $L, M, B, G, C, D$ are bounded, linear functionals on the appropriate spaces of continuous functions. They can be 
represented in the form

$$
\begin{aligned}
& L \varphi=\int_{0}^{h} d \eta(\tau) \varphi(-\tau), \quad M \varphi=\int_{0}^{h} d \mu(\tau) \varphi(-\tau), \quad C \varphi=\int_{0}^{h} d \kappa(\tau) \varphi(-\tau), \\
& B \xi=\int_{0}^{h} d \beta(\tau) \xi(-\tau), \quad G \xi=\int_{0}^{h} d \gamma(\tau) \xi(-\tau), \quad D \xi=\int_{0}^{h} d \delta(\tau) \xi(-\tau),
\end{aligned}
$$

for $\varphi \in \mathscr{C}\left[-h, T ; \mathbf{R}^{n}\right], \xi \in \mathscr{C}\left[-h, 0 ; \mathbf{R}^{m}\right]$ where $\eta, \mu, \beta, \gamma, \kappa, \delta$ are normalized matrix functions of bounded variation, that is they are constant for $\tau \geqslant h$, right continuous for $0<\tau<h$ and vanish for $\tau \leqslant 0$. Note that the expression $L x_{t}=$ $d \eta * x(t)$ makes sense as an $L^{2}$-function of $t$ if $x(\cdot) \in L^{2}\left[-h, 0 ; \mathbf{R}^{n}\right]$. In order to guarantee the existence and uniqueness for the solutions of (6.1) we will always assume that

$$
\mu(0)=\lim _{\tau \downarrow 0} \mu(\tau) .
$$

Given any control input $u(\cdot) \in L^{2}\left[-h, T ; \mathbf{R}^{m}\right]$ a function $x(\cdot) \in L^{2}\left[-h, 0 ; \mathbf{R}^{n}\right]$ is said to be a solution of (6.1) if the function

$$
q(t)=x(t)-M x_{t}-G u_{t}, \quad 0 \leqslant t \leqslant T
$$

is in $W^{1,2}\left[0, T ; \mathbf{R}^{n}\right)$ and satisfies $\dot{q}(t)=L x_{t}+B u_{t}$ for almost every $t \in[0, T]$. Under condition (6.2) it has been shown in $[\mathbf{4}, \mathbf{4 0}]$ that system (6.1), (6.3) admits a unique solution pair $x(\cdot) \in L^{2}\left[-h, T ; \mathbf{R}^{n}\right], q(\cdot) \in W^{1,2}\left[0, T ; \mathbf{R}^{n}\right]$ for every input $u(\cdot) \in L^{2}\left[0, T ; \mathbf{R}^{m}\right]$ and every initial condition of the form

$$
q(0)=\varphi^{0}, \quad x(\tau)=\varphi^{1}(\tau), \quad u(\tau)=\varphi^{2}(\tau), \quad-h \leqslant \tau<0,
$$

where

$$
\varphi=\left(\varphi^{0}, \varphi^{1}, \varphi^{2}\right) \in H=R^{n} \times L^{2}\left[-h, 0 ; \mathbf{R}^{n}\right] \times L^{2}\left[-h, 0 ; \mathbf{R}^{m}\right] .
$$

In this section we will be concerned with the problem of finding an abstract evolution equation which equivalently describes the solutions of (6.1). Such an evolution equation has been derived for neutral systems with state delays only $\left(G=0, B u_{t}=B_{0} u(t), C=0, D=0\right)$ by Burns, Herdman and Stech [4]. For retarded systems with input delays $(M=0, G=0, C=0, D=0)$ we refer to Ichikawa [20], Vinter and Kwong [44], Delfour [10], and for neutral systems with delays either in control or observation to Salamon [40]. Retarded systems with simultaneous delays in control and observation $(M=0, G=0, D=0)$ have been for the first time successfully treated in Pritchard and Salamon [34]. That approach, however, is not applicable if $D \neq 0$ the problem being "too much unboundedness" in the input and output operators. A state space approach for this class of systems ( $M=0, G=0$ ) has been developed recently by Delfour and Karrakchou [11] using the forcing function state concept which is due to Miller [31]. We use a different approach to derive an evolution equation representation for general system of the form (6.1).

In order to reformulate system (6.1) in the framework of $\$ 2.2$ we define

$$
\begin{aligned}
Z & =\left\{\varphi \in H \mid \varphi^{1} \in W^{1,2}\left[-h, 0 ; \mathbf{R}^{n}\right], \varphi^{2} \in W^{1,2}\left[-h, 0 ; \mathbf{R}^{m}\right],\right. \\
\left.\varphi^{0}=\varphi^{1}(0)-M \varphi^{1}-G \varphi^{2}\right\}, & \\
U & =\mathbf{R}^{m}, Y=\mathbf{R}^{p}, \\
\Delta \varphi & =\left(L \varphi^{1}-B \varphi^{2}, \dot{\varphi}^{1}, \dot{\varphi}^{2}\right), \quad \Gamma \varphi=\varphi^{2}(0), \quad K \varphi=C \varphi^{1}+D \varphi^{2} .
\end{aligned}
$$


THEOREM 6.1. Let the spaces $Z \subset H$ and the operators $\Delta \in \mathscr{L}(Z, H), \Gamma \in \mathscr{L}(Z, U)$, $K \in \mathscr{L}(Z, Y)$ be defined as above and let $\varphi \in Z, u(\cdot) \in W^{1,2}\left[0, T ; \mathbf{R}^{m}\right]$ satisfy $\varphi^{2}(0)=u(0)$. Furthermore, let $x(\cdot) \in L^{2}\left[-h, T ; \mathbf{R}^{n}\right], q(\cdot) \in W^{1,2}\left[0, T ; \mathbf{R}^{n}\right], y(\cdot) \in$ $L^{2}\left[0, T ; \mathbf{R}^{p}\right]$ be given and define

$$
\hat{x}(t)=\left(q(t), x_{t}, u_{t}\right) \in H,
$$

for $0 \leqslant t \leqslant T$. Then $x(t), q(t), y(t)$ satisfy (6.1), (6.3), (6.4) if and only if $\hat{x}(\cdot) \in$ $\mathscr{C}[0, T ; Z] \cap \mathscr{C}^{1}[0, T ; H]$ satisfies

$$
\left\{\begin{array}{l}
\frac{d}{d t} \hat{x}(t)=\Delta \hat{x}(t), \quad \hat{x}(0)=\varphi, \\
\Gamma \hat{x}(t)=u(t), \quad 0 \leqslant t \leqslant T, \\
y(t)=K \hat{x}(t) .
\end{array}\right.
$$

Furthermore, the BCS (6.6) is wellposed in the sense of Definition 2.10 .

Proof. If $x(t), w(t), y(t)$ satisfy (6.1), (6.3), (6.4), then $x(\cdot) \in W^{1,2}\left[-h, T ; \mathbf{R}^{n}\right]$ (see [40, Theorem 1.2.3]) and moreover $u(\cdot) \in W^{1,2}\left[-h, T ; \mathbf{R}^{m}\right]$. Now it follows from the shift property of the term $(6.5)$ that $\hat{x}(\cdot) \in \mathscr{C}[0, T ; Z] \cap \mathscr{C}^{1}[0, T ; H]$ and stantard arguments in the theory of FDEs (e.g. Bernier and Manitius [3], Salamon [40, Theorem 1.2.6]) show that $(d / d t) \hat{x}(t)=\Delta \hat{x}(t)$. The equations $u(t)=\Gamma \hat{x}$, $y(t)=K \hat{x}(t)$ are obviously satisfied. Therefore $\hat{x}(t)$ satisfies (6.6). The converse implication follows from the fact that the restriction of $\Delta$ to $\operatorname{ker} \Gamma$ is the infinitesimal generator of a strongly continuous semigroup [40, Theorem 1.2.6] and therefore the solutions of (6.6) are unique.

Combining Theorem 6.1 with the existence, uniqueness and continuous dependence results for the solutions of (6.1) (see e.g. [40, Theorem 1.2.3]) we obtain that the BCS (6.6) is wellposed in the sense of Definition 2.10.

In order to derive a satisfactory solution of the linear quadratic control problem we have to clarify the relation between the dual system of (6.6) and the transposed system of $(6.1)$. Following $[31,10,34,40,41]$ we write the transposed NFDE in the form

$$
\left\{\begin{aligned}
\dot{z}(t)= & -\int_{0}^{T-t} d \eta^{*}(\tau) z(t+\tau)-\int_{0}^{T-t} d \mu^{*}(\tau) \dot{z}(t+\tau) \\
& -\int_{0}^{T-t} d \kappa^{*}(\tau) v(t+\tau)-\psi^{1}(t-T), \\
w(t)= & \int_{0}^{T-t} d \beta^{*}(\tau) z(t+\tau)+\int_{0}^{T-t} d \gamma^{*}(\tau) \dot{z}(t+\tau) \\
& +\int_{0}^{T-t} d \delta^{*}(\tau) v(t+\tau)+\psi^{2}(t-T), \\
z(T)= & \psi^{0}, \quad t \leqslant T
\end{aligned}\right.
$$

where $\psi=\left(\psi^{0}, \psi^{1}, \psi^{2}\right) \in H$. The obvious existence, uniqueness and continuous dependence results hold for the solutions of (6.7) [40, Theorem 1.2.3]. The state of (6.7) at time $t \geqslant 0$ is the triple $\hat{z}(t)=\left(z(t), z^{t}, w^{t}\right) \in H$ where $z^{t} \in L^{2}\left[-h, 0 ; \mathbf{R}^{n}\right]$ 
and $w^{t} \in L^{2}\left[-h, 0 ; \mathbf{R}^{m}\right]$ are given by

$$
\left\{\begin{aligned}
z^{t}(s)= & \int_{-s}^{T-t-s} d \eta^{*}(\tau) z(t+s+\tau)+\int_{-s}^{T-t-s} d \mu^{*}(\tau) \dot{z}(t+s+\tau) \\
& +\int_{-s}^{T-t-s} d \kappa^{*}(\tau) v(t+s+\tau)+\psi^{1}(t+s-\tau) \\
w^{t}(s)= & \int_{-s}^{T-t-s} d \beta^{*}(\tau) z(t+s+\tau)+\int_{-s}^{T-t-s} d \gamma^{*}(\tau) \dot{z}(t+s+\tau) \\
& +\int_{-s}^{T-t-s} d \delta^{*}(\tau) v(t+s+\tau)+\psi^{2}(t+s-t)
\end{aligned}\right.
$$

for $-h \leqslant s \leqslant 0$. With this definition it follows from standard arguments in the theory of FDEs that the state $\hat{x}(t) \in H$ of the NFDE (6.1) and the state $\hat{z}(t) \in H$ of the NFDE (6.7) always satisfy equation (3.4) (see e.g. [41, 40, Theorem 2.3.5] or [Part II, Theorem 2.2]). Using this fact together with Corollary 3.4 we obtain the following result as an immediate consequence.

THEOREM 6.2. Let $\psi \in H, v(\cdot) \in L^{2}\left[0, T ; \mathbf{R}^{p}\right], z(\cdot) \in W^{1,2}\left[0, T ; \mathbf{R}^{n}\right], w(\cdot) \in$ $L^{2}\left[0, T ; \mathbf{R}^{m}\right]$ be given and let $\hat{z}(t)=\left(z(t), z^{t}, w^{t}\right) \in H$ be defined by (6.8). Then $z(t)$ and $w(t)$ satisfy (6.7) for almost every $t \in[0, T]$ if and only if $\hat{z}(\cdot) \in \mathscr{C}[0, T ; H] \cap$ $W^{1,2}\left[0, T ; Z^{*}\right]$ is the unique solution of the abstract POP

$$
\frac{d}{d t} \hat{z}(t)-\Gamma^{*} w(t)=-\Delta^{*} \hat{z}(t)-K^{*} v(t), \quad t \leqslant T, \hat{z}(T)=\psi .
$$

Furthermore, the POP (6.9) is wellposed in the sense of Definition 2.16.

We can now transform the BCS (6.6) and the POP (6.9) into their corresponding semigroup control system as in $\$ 2$ and then apply Theorem 5.1. Alternatively, we can use Corollary 5.8 direcly to obtain that the optimal control $u(\cdot)=u(\cdot ; \varphi) \in$ $L^{2}\left[0, T ; \mathbf{R}^{m}\right]$ which minimizes the cost functional (5.1) subject to $(6.1),(6.3),(6.4)$ is characterized by the transposed equation (6.7) with $\psi=0, w(t)=u(t), v(t)=y(t)$ (compare Karrakchou [22]).

7. Partial differential equations. The aim of this section is to clarify the relation between the framework in $\$ 2$ and the one developed by Lions [28] and Lions and Magenes [29] in their classical work. Although some of the material in this section is known, at some places things are presented in a slightly different way than usual. Therefore, we feel that a somewhat more elaborate discussion is appropriate.

The semigroup theoretic reformulation of boundary control systems is of course not new. Earlier work in this direction has been done for example by Balakrishnan [2], Washburn [45], Curtain and Pritchard [6], Lasiecka and Triggiani [24]. One of the important insights in $[\mathbf{2}, \mathbf{4 5}]$, is that the input operator is given, roughly speaking, by composing the infinitesimal generator with the solution operator of an elliptic problem. We find it convenient to take a slightly different route and introduce the input operator directly on the basis of a classical duality result (Theorem 7.1 below).

In order to avoid confusion we point out that throughout this section we denote by $V$ the space introduced by Lions and by $\Omega$ an open domain in $\mathbf{R}^{n}$. Furthermore, whenever the letter $\Delta$ appears in this section it will denote the operator of $\S 2.2$ and not the Laplacian. 
7.1. Parabolic systems. Consider the parabolic PDE with Dirichlet boundary control described by the equations

$$
\left\{\begin{array}{l}
\frac{\partial z}{\partial t}(x, t)+\sum_{|\rho|,|\sigma| \leqslant m}(-1)^{|\rho|} D^{\rho}\left(a_{\rho \sigma} D^{\sigma} z\right)(x, t)=0, \quad x \in \Omega, t>0, \\
D_{\nu}^{j} z(x, t)=u_{j}(x, t), \quad x \in \partial \Omega, t>0, j=0,1, \ldots, m-1,
\end{array}\right.
$$

on a bounded, open domain $\Omega \subset \mathbf{R}^{n}$ whose boundary $\partial \Omega$ is a compact orientable $\mathscr{C}^{\infty}$-manifold. We assume that the coefficients $a_{\rho \sigma}(x)$ are in $\mathscr{C}^{\infty}(\bar{\Omega})$ and satisfy the uniform ellipticity condition

$$
\sum_{|\rho|,|\sigma| \leqslant m} a_{\rho \sigma}(x) \xi^{\rho} \xi^{\sigma} \geqslant c|\xi|^{2}, \quad x \in \Omega, \xi \in R^{n},
$$

for some constant $c>0$. The functions $u_{j}(x, t)$ are understood as the control inputs and it is the aim of this section to show how system (7.1) fits into the framework of $\$ 2$.

Following Lions [28] and Lions and Magenes [29] we first introduce the spaces $V=H_{0}^{m}(\Omega), H=L^{2}(\Omega), V^{*}=H^{-m}(\Omega)$ so that $V \subset H \subset V^{*}$ with continuous, dense injections and we define the bilinear form $a(\cdot, \cdot)$ on $H^{m}(\Omega)$ by

$$
a(\psi, \varphi)=\sum_{|\rho|,|\sigma| \leqslant m} \int_{\Omega} a_{\rho \sigma}(x) D^{\rho} \psi(x) D^{\sigma} \varphi(x) d x
$$

for $\varphi, \psi \in H^{m}(\Omega)$. Furthermore, we introduce the differential operators $L$ and $L^{*}$ from $H^{2 m}(\Omega)$ into $L^{2}(\Omega)$ by

$$
\begin{aligned}
L \varphi & =\sum_{|\rho|,|\sigma| \leqslant m}(-1)^{|\rho|} D^{\rho}\left(a_{\rho \sigma} D^{\sigma} \varphi\right), \\
L^{*} \psi & =\sum_{|\rho|,|\sigma| \leqslant m}(-1)^{|\sigma|} D^{\sigma}\left(a_{\rho \sigma} D^{\rho} \psi\right)
\end{aligned}
$$

for $\varphi, \psi \in H^{2 m}(\Omega)$. Then the following basic duality result plays a centrol role in this section. For the proof we refer to Folland [15, p. 288] in connection with the trace theorem.

THEOREM 7.1. (i) There exist differential operators $B_{j}^{*}$ of order $2 m-1-j$ for $j=0, \ldots, m-1$, defined in a neighborhood of $\partial \Omega$, such that the following equation holds for $\varphi \in H^{m}(\Omega), \psi \in H^{2 m}(\Omega)$,

$$
\left\langle L^{*} \psi, \varphi\right\rangle_{L^{2}(\Omega)}=a(\psi, \varphi)+\sum_{0}^{m-1} \int_{\partial \Omega} B_{j}^{*} \psi(x) D_{\nu}^{j} \varphi(x) d S(x) .
$$

The highest order term of $B_{j}^{*}$ is of the form $b_{j}(x) D_{\nu}^{2 m-1-j}+D_{\tau}^{2 m-1-j}$ where $b_{j}(\cdot) \in \mathscr{C}^{\infty}(\partial \Omega)$ is bounded away from zero and $D_{\tau}^{2 m-1-j}$ is a tangential differential operator on $\partial \Omega$ of order at most $2 m-1-j$.

(ii) For $\varphi \in H^{2 m}(\Omega)$ and $\psi \in H_{0}^{m}(\Omega)$ we have $\langle\psi, L \varphi\rangle=a(\psi, \varphi)$.

This theorem shows that every classical solution of the parabolic PDE (7.1) is a weak solution in the following sense. 
Definition 7.2 (WeAK SOlution). Let $u_{j}(\cdot) \in L^{2}\left[0, T ; L^{2}(\partial \Omega)\right]$ be given. Then $a$ function $z(\cdot) \in L^{2}\left[0, T ; L^{2}(\Omega)\right]$ is said to be a weak solution of $(7.1)$ if $\langle\psi, z(\cdot)\rangle \in$ $W^{1,2}[0, T]$ for every $\psi \in H^{2 m}(\Omega) \cap H_{0}^{m}(\Omega)$ and the following equation holds for almost every $t \in[0, T]$

$$
\frac{d}{d t}\langle\psi, z(t)\rangle+\left\langle L^{*} \psi, z(t)\right\rangle=\sum_{0}^{m-1}\left\langle B_{j}^{*} \psi, u_{j}(t)\right\rangle_{L^{2}(\partial \Omega)} .
$$

The main step toward the existence and uniqueness result for weak solutions is the Gårding inequality

$$
a(\varphi, \varphi) \geqslant \varepsilon\|\varphi\|_{V}^{2}-\omega\|\varphi\|_{H}^{2}, \quad \varphi \in V,
$$

which follows from the uniform ellipticity (7.2) (see for example Folland [15, p. 309]). This inequality in connection with standard results in semigroup theory shows that the operator $A: V \rightarrow V^{*}$ defined by

$$
\langle\psi, A \varphi\rangle=-a(\psi, \varphi), \quad \varphi, \psi \in V,
$$

is the infinitesimal generator of an analytic semigroup $S(t) \in \mathscr{L}\left(V^{*}\right)$ (see for example Pazy [32], Friedman [16]). Furthermore, it follows from Rellich's lemma that $A$ has a compact resolvent operator. The restriction of $A$ to $\mathscr{D}(A)=\{\varphi \in$ $V \mid A \varphi \in H\}$ is the generator of the restricted semigroup $S(t) \in \mathscr{L}(H)$. A classical result in the $L^{2}$ theory for elliptic boundary value problems shows that the domain of this restriction is given by

$$
W=\mathscr{D}(A)=\{\varphi \in V \mid A \varphi \in H\}=H^{2 m}(\Omega) \cap H_{0}^{m}(\Omega)
$$

(Agmon, Douglis and Nirenberg [1], Friedman [16]). Moreover, Theorem 7.1 shows that $A \varphi=-L \varphi, \varphi \in W$. Now the trace theorem allows us to understand the boundary operators $B_{j}^{*}$ in Theorem 7.1 as bounded linear transformations from $W$ into $U=L^{2}(\partial \Omega)$. Hence $B_{j} \in \mathscr{L}\left(U ; W^{*}\right)$ and we can rewrite (7.3) into the abstract Cauchy problem

$$
\dot{z}(t)=A z(t)+\sum_{0}^{m-1} B_{j} u_{j}(t), \quad z(0)=z_{0} \in V^{*} .
$$

For all $z_{0} \in V^{*}$ and all $u_{j}(\cdot) \in L^{2}[0, T ; U]$ the corresponding solution of (7.5) is given by the variation-of-constants formula

$$
z\left(t ; z_{0}, u\right)=S(t) z_{0}+\sum_{0}^{m-1} \int_{0}^{t} S(t-s) B_{j} u_{j}(s) d s .
$$

It follows from results by DaPrato [8], Balakrishnan [2], Washburn [45], Lasiecka [23] that this solution always lies in

$$
z\left(\cdot ; z_{0}, u\right) \in L^{2}[0, T ; H] \cap \mathscr{C}\left[0, T ; V^{*}\right] \cap W^{1,2}\left[0, T ; W^{*}\right]
$$

and depends in these spaces continuously on $z_{0} \in V^{*}$ and $u_{j}(\cdot) \in L^{2}[0, T ; U]$, $j=0, \ldots, m-1$. This proves the existence, uniqueness and continuous dependence for the weak solutions of (7.1). In other words, the SCS (7.5) is wellposed in the state space $V^{*}$ in the sense of Definition 2.4, if the output is defined through any bounded operator on $H$. 
In order to transform the SCS (7.5) into an abstract BCS with $V^{*}$ as a state space we have to make sure that the input operators $B_{j} \in \mathscr{L}\left(U, W^{*}\right)$ are strictly unbounded with respect to this space.

LEMMA 7.3. $\sum_{0}^{m-1} B_{j} u_{j} \in V^{*} \Rightarrow u_{0}=u_{1}=\cdots=u_{m-1}=0$.

Proof. Suppose that not all the $u_{j}$ vanish identically and let $k \in\{0, \ldots, m-1\}$ be the smallest index with $u_{k} \not \equiv 0$. Choose $v(\cdot) \in \mathscr{C}^{\infty}(\partial \Omega)$ such that $\left\langle v, u_{k}\right\rangle=1$. Let $\nu(x), x \in \partial \Omega$, denote the (global, smooth) outward unit normal vector. Then the neighborhood $V_{\varepsilon}=\{x+t \nu(x) \mid x \in \partial \Omega,-\varepsilon<t<\varepsilon\}$ is diffeomorphic to $\partial \Omega \times$ $(-\varepsilon, \varepsilon)$ if $\varepsilon$ is sufficiently small. Finally, let $\zeta(\cdot) \in \mathscr{C}^{\infty}(R ;[0,1])$ satisfy $\zeta(t)=1$ for $|t| \leqslant 1 / 4$ and $\zeta(t)=0$ for $|t| \geqslant 1 / 2$. Then we define $\psi_{\varepsilon} \in \mathscr{C}^{\infty}(\bar{\Omega})$ by

$$
\psi_{\varepsilon}(x+t \nu(x))=\zeta\left(\frac{t}{\varepsilon}\right) \frac{t^{2 m-1-k}}{(2 m-1-k) !} \frac{v(x)}{b_{k}(x)}, \quad x \in \partial \Omega,-\varepsilon<t \leqslant 0,
$$

and $\psi_{\varepsilon}(x)=0$ for $x \in \Omega \backslash V_{\varepsilon}$ (compare Folland [15, p. 294]). Then $B_{j}^{*} \psi_{\varepsilon}=0$ for $j>k$ and $B_{k}^{*} \psi_{\varepsilon}=v$. This implies

$$
\left\langle\psi_{\varepsilon}, \sum_{0}^{m-1} B_{j} u_{j}\right\rangle=\sum_{0}^{m-1}\left\langle B_{j}^{*} \psi_{\varepsilon}, u_{j}\right\rangle=\left\langle v, u_{k}\right\rangle=1
$$

Since $\psi_{\varepsilon} \in H_{0}^{m}(\Omega)$ and $\left\|\psi_{\varepsilon}\right\|_{H^{m}(\Omega)}$ tends to zero as $\varepsilon$ approaches zero, we conclude that $\sum_{0}^{m-1} B_{j} u_{j} \notin V^{*}$.

The previous lemma allows us to introduce the space

$$
Z=\left\{\varphi \in H \mid A \varphi \in V^{*}+\sum_{0}^{m-1} \text { range } B_{j}\right\}
$$

and operators $\Delta: Z \rightarrow V^{*}, \Gamma_{j}: Z \rightarrow U$ such that

$$
A \varphi+\sum_{0}^{m-1} B_{j} \Gamma_{j} \varphi=\Delta \varphi, \quad \varphi \in Z .
$$

Then $Z$ becomes a Hilbert space if we define

$$
\|\varphi\|_{Z}^{2}=\|\varphi\|_{H}^{2}+\|\Delta \varphi\|_{V^{*}}^{2}+\sum_{0}^{m-1}\left\|\Gamma_{j} \varphi\right\|_{U}^{2}
$$

Now the results of $\S 2.2$ show that the SCS (7.5) is equivalent to the BCS

$$
\begin{cases}\dot{z}(t)=\Delta z(t), & t \geqslant 0, z(0)=z_{0} \in Z \\ \Gamma_{j} z(t)=u_{j}(t), & j=0, \ldots, m-1 .\end{cases}
$$

More precisely, the BCS (7.8) is wellposed in the state space $V^{*}$ (Proposition 2.11) and for all $z_{0} \in Z$ and all $u_{j}(\cdot) \in W^{1,2}\left[0, T ; L^{2}(\partial \Omega)\right]$ with $\Gamma_{j} z_{0}=u_{j}(0)$ the function $z\left(\cdot ; z_{0}, u\right)$ defined by (7.6) is in $\mathscr{C}[0, T ; Z] \cap \mathscr{C}^{1}\left[0, T ; V^{*}\right]$ and satisfies (7.8) (Proposition 2.8). Moreover, the definition of $Z$ shows that every solution $z(\cdot) \in$ $L^{2}[0, T ; H] \cap W^{1,2}\left[0, T ; V^{*}\right]$ of the $\operatorname{SCS}(7.5)$ is in $L^{2}[0, T ; Z]$ and also satisfies (7.7). 
LEMmA 7.4. (i) $H^{m}(\Omega) \subset Z$ with a continuous, dense injection and

$$
\begin{gathered}
\langle\psi, \Delta \varphi\rangle=-a(\psi, \varphi), \quad \varphi \in H^{m}(\Omega), \psi \in H_{0}^{m}(\Omega), \\
\Gamma_{j} \varphi=D_{\nu}^{j} \varphi, \quad \varphi \in H^{m}(\Omega), j=0, \ldots, m-1 .
\end{gathered}
$$

In particular, $\Delta \varphi=-L \varphi$ for $\varphi \in H^{2 m}(\Omega)$.

(ii) Let $u_{j} \in L^{2}(\partial \Omega)$ be given and suppose that $\varphi=\sum_{0}^{m-1}(\mu I-A)^{-1} B_{j} u_{j} \in$ $H^{m}(\Omega)$. Then

$$
\begin{cases}a(\psi, \varphi)+\mu\langle\psi, \varphi\rangle=0, & \psi \in H_{0}^{m}(\Omega), \\ D_{\nu}^{j} \varphi=u_{j}, & j=0,1, \ldots, m-1 .\end{cases}
$$

(iii) If $\Phi \in H^{m}(\Omega)$ then $\varphi=\sum_{0}^{m-1}(\mu I-A)^{-1} B_{j} D_{\nu}^{j} \Phi \in H^{m}(\Omega)$ and (7.11) holds with $u_{j}=D_{\nu}^{j} \Phi$.

Proof. Let $\varphi \in H^{m}(\Omega)$ be given and define $\Phi \in V^{*}$ by $\langle\psi, \Phi\rangle=-a(\psi, \varphi)$ for $\psi \in H_{0}^{m}(\Omega)$. Then it follows from Theorem 7.1 that the following equation holds for $\psi \in H^{2 m}(\Omega) \cap H_{0}^{m}(\Omega)$ :

$$
\left\langle\psi, A \varphi+\sum_{0}^{m-1} B_{j} D_{\nu}^{j} \varphi\right\rangle_{W, W^{*}}=-\left\langle L^{*} \psi, \varphi\right\rangle_{H}+\sum_{0}^{m-1}\left\langle B_{j}^{*} \psi, D_{\nu}^{j} \varphi\right\rangle_{U}=\langle\psi, \Phi\rangle_{V, V^{*}}
$$

and hence

$$
A \varphi+\sum_{0}^{m-1} B_{j} D_{\nu}^{j} \varphi=\Phi \in V^{*} .
$$

By equation (7.7) and Lemma 7.3, this implies $\varphi \in Z, \Delta \varphi=\Phi, \Gamma_{j} \varphi=D_{\nu}^{j} \varphi$. Thus we have proved that $H^{m}(\Omega) \subset Z$ and (7.9), (7.10) hold. It follows from (7.9) and (7.10) that there exists a constant $c>0$ such that $\|\varphi\|_{Z} \leqslant c\|\varphi\|_{H^{m}}$ for all $\varphi \in H^{m}(\Omega)$ and hence the injection of $H^{m}(\Omega)$ into $Z$ is continuous.

This proves all the assertions of statement (i) except for the density of $H^{m}(\Omega)$ in $Z$. Statement (ii) follows now immediately from Proposition 2.8(i). In order to prove statement (iii), let $\Phi \in H^{m}(\Omega)$ be given and define $\varphi=\sum_{0}^{m-1}(\mu I-A)^{-1} B_{j} \Gamma_{j} \Phi$. Then it follows again from Proposition 2.8 that $\varphi \in Z$ and $\Delta \varphi=\mu \varphi, \Gamma_{j} \varphi=\Gamma_{j} \Phi$. Therefore we get from equation (7.7) that $A(\varphi-\Phi)=\Delta \varphi-\Delta \Phi \in V^{*}$ and hence $\varphi-\Phi \in H_{0}^{m}(\Omega)$. This proves statement (iii).

Now let $\varphi \in Z$ be given and observe that

$$
\varphi-\sum_{0}^{m-1}(\mu I-A)^{-1} B_{j} \Gamma_{j} \varphi=(\mu I-A)^{-1}(\mu \varphi-\Delta \varphi) \in H_{0}^{m}(\Omega) .
$$

Moreover, choose $u_{j}^{n} \in \mathscr{C}^{\infty}(\partial \Omega)$ converging to $\Gamma_{j} \varphi$ in $L^{2}(\partial \Omega)$. Then there exists a $\Phi^{n} \in \mathscr{C}^{\infty}(\bar{\Omega})$ with $\Gamma_{j} \Phi^{n}=u_{j}^{n}$ for $j=0, \ldots, m-1$ (Folland [15, p. 294]). Hence it follows from statement (iii) that

$$
\varphi^{n}=\varphi+\sum_{0}^{m-1}(\mu I-A)^{-1} B_{j}\left(u_{j}^{n}-\Gamma_{j} \varphi\right) \in H^{m}(\Omega) .
$$


Furthermore, $\varphi^{n}$ converges to $\varphi$ in $Z$ since $\Gamma_{j} \varphi^{n}=u_{j}^{n}$ and

$$
\Delta\left(\varphi^{n}-\varphi\right)=\sum_{0}^{m-1} \mu(\mu I-A)^{-1} B_{j}\left(u_{j}^{n}-\Gamma_{j} \varphi\right) .
$$

Therefore $H^{m}(\Omega)$ is dense in $Z$.

REMARKS 7.5. (i) The results of this section indicate that $Z$ is the natural space for studying the solutions of both the parabolic PDE (7.1) and the associated elliptic boundary value problem (7.11). It follows from Lemma 7.4 that $Z$ is the completion of $\mathscr{C}^{\infty}(\bar{\Omega})$ with respect to the norm

$$
\|\varphi\|_{Z}^{2}=\|\varphi\|_{L^{2}(\Omega)}^{2}+\|L \varphi\|_{H^{-m}(\Omega)}^{2}+\sum_{0}^{m-1}\left\|D_{\nu}^{j} \varphi\right\|_{L^{2}(\partial \Omega)}^{2} .
$$

(ii) Let us define $B \in \mathscr{L}\left(U^{m}, W^{*}\right)$ by $B u=\sum_{0}^{m-1} B_{j} u_{j}$ for $u=\left(u_{0}, \ldots, u_{m-1}\right) \in$ $U^{m}$. Then it follows from Lemma 7.4 that the operator $(\mu I-A)^{-1} B \in \mathscr{L}\left(U^{m}, Z\right)$ is the extended solution operator for the elliptic boundary value problem (7.11). This relates our results to those by Balakrishnan [2], Washburn [45].

(iii) If $u \in U^{m}$ then it is easy to see that $\varphi=(\mu I-A)^{-1} B u \in L^{2}(\Omega)$ satisfies $L \varphi+\mu \varphi=0$ in the distributional sense. Hence it follows from the local regularity theorem for elliptic operators (see for example Folland [15, p. 269]) that $\varphi \in \mathscr{C}_{\text {loc }}^{\infty}(\Omega)$. This shows that $Z \subset H_{\mathrm{loc}}^{m}(\Omega)$.

7.2. Hyperbolic systems. Consider the hyperbolic PDE with Dirichlet boundary control described by the equations

$$
\left\{\begin{array}{l}
\frac{\partial^{2} z}{\partial t^{2}}(x, t)+\sum_{|\rho|,|\sigma| \leqslant m}(-1)^{|\rho|} D^{\rho}\left(a_{\rho \sigma} D^{\sigma} z\right)(x, t)=0, \quad x \in \Omega, t>0 \\
D_{\nu}^{j} z(x, t)=u_{j}(x, t), \quad x \in \partial \Omega, t>0, j=0, \ldots, m-1, \\
y(t)=C \frac{\partial z}{\partial t}(\cdot, t), \quad t>0
\end{array}\right.
$$

where $\Omega \subset R^{n}$ and $a_{\rho \sigma}(x)$ have the same properties as in the previous section and $C$ is a bounded linear operator from $H=L^{2}(\Omega)$ into the Hilbert space $Y$. Also the spaces $U=L^{2}(\Omega), W=H^{2 m}(\Omega) \cap H_{0}^{m}(\Omega), V=H_{0}^{m}(\Omega)$, the bilinear form $a(\cdot, \cdot)$ on $H^{m}(\Omega)$ and the operators $L, L^{*} \in \mathscr{L}\left(H^{2 m}(\Omega), L^{2}(\Omega)\right), B_{j} \in \mathscr{L}\left(U, W^{*}\right), A \in$ $\mathscr{L}(W, H) \cap \mathscr{L}\left(V, V^{*}\right) \cap \mathscr{L}\left(H, W^{*}\right)$ are defined as in $\S 7.1$. Then it follows again from Theorem 7.1 that every classical solution of (7.12) is a weak solution in the following sense.

Definition 7.6 (WeAK Solution). Let $u_{j}(\cdot) \in L^{2}\left[0, T ; L^{2}(\partial \Omega)\right]$ be given. Then a function $z(\cdot) \in \mathscr{C}\left[0, T ; L^{2}(\Omega)\right] \cap \mathscr{C}^{1}\left[0, T ; H^{-m}(\Omega)\right]$ is said to be a weak solution of (7.12) if $\langle\psi, z(\cdot)\rangle \in W^{2,2}[0, T]$ for every $\psi \in H^{2 m}(\Omega) \cap H_{0}^{m}(\Omega)$ and the following equation holds for almost every $t \in[0, T]$ :

$$
\frac{d^{2}}{d t^{2}}\langle\psi, z(t)\rangle+\left\langle L^{*} \psi, z(t)\right\rangle=\sum_{0}^{m-1}\left\langle B_{j}^{*} \psi, u_{j}(t)\right\rangle_{L^{2}(\partial \Omega)} .
$$


This means that $z(\cdot) \in \mathscr{C}[0, T ; H] \cap \mathscr{C}^{1}\left[0, T ; V^{*}\right] \cap W^{2,2}\left[0, T ; W^{*}\right]$ satisfies the second order abstract Cauchy problem

$$
\begin{gathered}
\ddot{z}(t)=A x(t)+B u(t), \quad t>0, \\
z(0)=\varphi^{0} \in H, \quad \dot{z}(0)=\varphi^{1} \in V^{*},
\end{gathered}
$$

where $u(t) \in \mathscr{U}=U^{m}$ and $B \in \mathscr{L}\left(\mathscr{U}, W^{*}\right)$ is defined as in Remark 7.5(ii). If $z(\cdot) \in \mathscr{C}^{1}[0, T ; H]$ then the output of the system is given by

$$
y(t)=\frac{d}{d t} C z(t), \quad t>0 .
$$

In order to rewrite system (7.14) into a first order SCS as in $\$ 2.1$ we introduce the spaces $\mathscr{W}=V \times H, \mathscr{H}=H \times V^{*}, \mathscr{V}=V^{*} \times W^{*}$ so that $\mathscr{W} \subset \mathscr{H} \subset \mathscr{V}$ with continuous, dense injections. We consider on each of these spaces the standard inner product and identify none of them with its respective dual. We also introduce the operators $\mathscr{C} \in \mathscr{L}(\mathscr{W}, \mathscr{Y}), \quad \mathscr{A} \in \mathscr{L}(\mathscr{W}, \mathscr{H}) \cap \mathscr{L}(\mathscr{H}, \mathscr{V}), \quad \mathscr{B} \in \mathscr{L}(\mathscr{U}, \mathscr{V}), \quad \mathscr{T}_{\mu} \in$ $\mathscr{L}(\mathscr{U}, \mathscr{Y})$ by

$$
\begin{gathered}
\mathscr{A}=\left[\begin{array}{ll}
0 & I \\
A & 0
\end{array}\right], \quad \mathscr{B}=\left[\begin{array}{l}
0 \\
B
\end{array}\right], \\
\mathscr{C}=\left[\begin{array}{ll}
0 & C
\end{array}\right], \quad \mathscr{T}_{\mu}=\mu C\left(\mu^{2} I-A\right)^{-1} B .
\end{gathered}
$$

Observe that the range of $(\mu I-\mathscr{A})^{-1} \mathscr{B}$ is always contained in $H \times H$, that $\mathscr{C}=$ $\left[\begin{array}{ll}0 & C\end{array}\right]$ extends naturally to a bounded operator on this space and that $\mathscr{T}_{\mu}$ is given by the composition of these operators. Now for every weak solution $z(t)$ of (7.12) the function $\hat{z}(\cdot)=(z(\cdot), \dot{z}(\cdot)) \in \mathscr{C}[0, T ; \mathscr{H}] \cap W^{1,2}[0, T ; \mathscr{V}]$ satisfies the abstract SCS

$$
\left\{\begin{array}{l}
\frac{d}{d t} \hat{z}(t)=\mathscr{A} \hat{z}(t)+\mathscr{B} u(t), \quad t \geqslant 0, \hat{z}(0)=\varphi \in \mathscr{H}, \\
y(t)=\mathscr{C}(\mu I-\mathscr{A})^{-1}\left(\mu \hat{z}(t)-\frac{d}{d t} \hat{z}(t)\right)+\mathscr{T}_{\mu} u(t),
\end{array}\right.
$$

and vice versa. The equation for the output, of course, requires that $\hat{z}(t) \in$ $W^{1,2}[0, T ; \mathscr{H}]$. It can be written in the simple form $y(t)=\mathscr{C} \hat{z}(t)$ if $\mathscr{C}$ is understood as the extended operator on $H \times H$. However, for some purposes it is convenient to keep (7.15) in its more complicated form. In particular, the duality relations can be derived in a straightforward way and the results of $\S \S 4$ and 5 can be applied directly.

It requires only the Gårding inequality (7.4) to establish the hypotheses (S0) and (S1) of $\S 2.1$ for the SCS (7.15). In fact, it follows from the Hille-Yoshida-Phillips theorem that $\mathscr{A}$ is the infinitesimal generator of a strongly continuous group $\mathscr{S}(t) \in \mathscr{L}(\mathscr{W}) \cap \mathscr{L}(\mathscr{H}) \cap \mathscr{L}(\mathscr{V})$. This proves the uniqueness for the weak solutions of (7.12) and their existence if $\varphi=\left(\varphi^{0}, \varphi^{1}\right) \in \mathscr{H}$ and $u_{j}(\cdot) \in$ $W^{1,2}\left[0, T ; L^{2}(\partial \Omega)\right]$. We denote the weak solution of (7.12), respectively (7.14), always by $z(t ; \varphi, u)$ and it is given by the first component of

$$
\hat{z}(t ; \varphi, u)=\mathscr{S}(t) \varphi+\int_{0}^{t} \mathscr{S}(t-s) \mathscr{B} u(s) d s \in \mathscr{H} .
$$


If $z(\cdot ; \varphi, u) \in \mathscr{C}^{1}[0, T ; H]$ then the corresponding output of (7.12), respectively (7.14) or (7.15), will be denoted by $y(t ; \varphi, u)$. If the SCS (7.15) is wellposed then the same notation extends to arbitrary $u(\cdot) \in L^{2}[0, T ; \mathscr{U}]$. In this case it follows from the usual density and continuous dependence arguments that the equation

$$
C z(t ; \varphi, u)=C \varphi^{0}+\int_{0}^{t} y(s ; \varphi, u) d s
$$

holds for all $\varphi \in \mathscr{H}, u(\cdot) \in L^{2}[0, T ; \mathscr{U}], t \geqslant 0$.

Now let $Z \subset H, \Delta \in \mathscr{L}\left(Z, V^{*}\right), \Gamma_{j} \in \mathscr{L}(Z, U)$ be given as in $\S 7.1$ and define $\Gamma \in \mathscr{L}(Z, \mathscr{U})$ by $\Gamma \varphi=\left(\Gamma_{0} \varphi, \ldots, \Gamma_{m-1} \varphi\right)$ for $\varphi \in Z$. Then we get

$$
\mathscr{Z}=\{\varphi \in \mathscr{H} \mid \mathscr{A} \varphi \in \mathscr{H}+\text { range } \mathscr{B}\}=Z \times H
$$

and the SCS (7.15) is related to the BCS

$$
\left\{\begin{array}{l}
\frac{d}{d t} \hat{z}(t)=\left(\begin{array}{cc}
0 & I \\
\Delta & 0
\end{array}\right) \hat{z}(t), \quad \hat{z}(0)=\varphi \in \mathscr{Z}, \\
{\left[\begin{array}{ll}
\Gamma & 0] \hat{z} \\
y(t)=u(t), \\
y(t)=\left[\begin{array}{ll}
0 & C
\end{array}\right] \hat{z}(t), \quad t \geqslant 0
\end{array}\right.}
\end{array}\right.
$$

as in $\S 2.2$. This means that $\hat{z}(\cdot) \in \mathscr{C}^{1}[0, T ; \mathscr{H}]$ satisfies $(7.15)$ if and only if $\hat{z}(\cdot) \in \mathscr{C}[0, T ; \mathscr{Z}]$ and $(7.17)$ holds.

We point out that everything remains the same if $\mathscr{U}$ is an arbitrary Hilbert space and $B \in \mathscr{L}\left(\mathscr{U}, W^{*}\right)$ is injective and strictly unbounded with respect to $V^{*}$ which we will assume from now on. In that context it is interesting to state explicitly the consequences of the perturbation result in $\S 4$. For this purpose we denote by $\mathscr{B}(T) \in \mathscr{L}\left(L^{2}[0, T ; \mathscr{U}] ; \mathscr{H}\right), \quad \mathscr{C}(T) \in \mathscr{L}\left(\mathscr{H}, L^{2}[0, T ; \mathscr{Y}]\right) \quad$ and $\quad \mathscr{T}(T) \in$ $\mathscr{L}\left(L^{2}[0, T ; \mathscr{U}], L^{2}[0, T ; \mathscr{Y}]\right)$ for $T>0$ the operators introduced in $\S 2.1$.

Corollary 7.7. Suppose that the SCS (7.15) is wellposed in the sense of Definition 2.4, that $\mathscr{Y}=\mathscr{U}$ and that $I-\mathscr{T}(t)$ is invertible for $t>0$. Then the following statements hold.

(i) The operators $\mathscr{S}_{F}(t)=\mathscr{S}(t)+\mathscr{B}(t)[I-\mathscr{T}(t)]^{-1} \mathscr{C}(t) \in \mathscr{L}(\mathscr{H})$ define a strongly continuous semigroup whose infinitesimal generator will be denoted by $\mathscr{A}_{F}$.

(ii) The closed loop input

$$
u(\cdot)=u_{F}(\cdot ; \varphi)=[I-\mathscr{T}(T)]^{-1} \mathscr{C}(T) \varphi \in L^{2}[0, T ; \mathscr{U}]
$$

and the first conponent

$$
z(\cdot)=z_{F}(\cdot ; \varphi) \in \mathscr{C}[0, T ; H] \cap \mathscr{C}^{1}\left[0, T ; V^{*}\right] \cap W^{2,2}\left[0, T ; W^{*}\right]
$$

of $\mathscr{S}(t) \varphi$ are the unique solutions of the feedback system

$$
\left\{\begin{array}{l}
\ddot{z}(t)=A x(t)+B u(t) \in W^{*}, \quad z(0)=\varphi^{0} \in H, \dot{z}(0)=\varphi^{1} \in V^{*}, \\
u(t)=\frac{d}{d t} C z(t), \quad t \geqslant 0,
\end{array}\right.
$$

whenever $\varphi \in H$. Furthermore $\mathscr{S}_{F}(t) \varphi=(z(t), \dot{z}(t))$ for all $t \geqslant 0$.

(iii) $\mathscr{W}_{F}=\left\{\varphi \in H \times H \mid A \varphi^{0}+B C \varphi^{1} \in V^{*}\right\}=\left\{\varphi \in Z \times H \mid \Gamma \varphi^{0}=C \varphi^{1}\right\} \subset$ $\mathscr{D}\left(\mathscr{A}_{F}\right)$ is invariant under $\mathscr{S}_{F}(t)$. Moreover, if $\varphi \in \mathscr{W}_{F}$ then $\mathscr{A}_{F} \varphi=\left(\varphi^{1}, A \varphi^{0}+\right.$ $\left.B C \varphi^{1}\right)=\left(\varphi^{1}, \Delta \varphi^{0}\right)$ and $z_{F}(\cdot ; \varphi) \in \mathscr{C}[0, T ; Z] \cap \mathscr{C}^{1}[0, T ; H] \cap \mathscr{C}^{2}\left[0, T ; V^{*}\right]$, 
$u_{F}(\cdot ; \varphi) \in W^{1,2}[0, T ; \mathscr{U}] \quad$ with $\dot{z}_{F}(t ; \varphi)=z_{F}\left(t ; \mathscr{A}_{F} \varphi\right), \quad \dot{u}_{F}(t ; \varphi)=u_{F}\left(t ; \mathscr{A}_{F} \varphi\right)$, $u_{F}(t ; \varphi)=\Gamma z_{F}(t ; \varphi)=C z_{F}(t ; \varphi)$.

(iv) If either $\mathscr{U}$ is finite dimensional or $C \in \mathscr{L}\left(V^{*}, \mathscr{Y}\right)$, then $\mathscr{D}\left(\mathscr{A}_{F}\right)=\mathscr{W}_{F}$.

We consider the dual system of the SCS (7.15) in the dual spaces $\mathscr{V}^{*}=V \times W$, $\mathscr{H}^{*}=H \times V, \mathscr{W}^{*}=V^{*} \times H$. We identify only the spaces $\mathscr{U}$ and $\mathscr{Y}$ with their respective dual so that the operators $\mathscr{A}^{*} \in \mathscr{L}\left(\mathscr{V}^{*}, \mathscr{H}^{*}\right) \cap \mathscr{L}\left(\mathscr{H}^{*}, \mathscr{W}^{*}\right), \mathscr{C}^{*} \in$ $\mathscr{L}\left(\mathscr{Y}, \mathscr{W}^{*}\right), \mathscr{B}^{*} \in \mathscr{L}\left(\mathscr{V}^{*}, \mathscr{U}\right), \mathscr{T}_{\mu}^{*} \in \mathscr{L}(\mathscr{Y}, \mathscr{U})$ are given by

$$
\begin{array}{ll}
\mathscr{A}^{*}=\left(\begin{array}{cc}
0 & A^{*} \\
I & 0
\end{array}\right), & \mathscr{C}^{*}=\left(\begin{array}{c}
0 \\
C^{*}
\end{array}\right), \\
\mathscr{B}^{*}=\left[\begin{array}{ll}
0 & B^{*}
\end{array}\right], & \mathscr{T}_{\mu}^{*}=\mu B^{*}\left(\mu^{2} I-A^{*}\right)^{-1} C^{*} .
\end{array}
$$

Observe that the range of $\left(\mu I-\mathscr{A}^{*}\right)^{-1}$ is always contained in $H \times W$, that $\mathscr{B}^{*}=\left[\begin{array}{ll}0 & B^{*}\end{array}\right]$ extends naturally to a bounded operator on this space and that $\mathscr{T}_{\mu}^{*}$ is given by the composition of these operators. Now the dual SCS in the time reverse form is described by the equations

$$
\left\{\begin{array}{l}
\frac{d}{d t} \hat{w}(t)=-\mathscr{A}^{*} \hat{w}(t)-\mathscr{C}^{*} y(t), \quad \hat{w}(T)=\psi \in \mathscr{H}^{*}, \\
u(t)=-\mathscr{B}^{*}\left(\mu I-\mathscr{A}^{*}\right)\left(\mu \hat{w}(t)-\frac{d}{d t} \hat{w}(t)\right)-\mathscr{T}_{\mu}^{*} y(t), \quad t \leqslant T .
\end{array}\right.
$$

The equation for the output requires that $\hat{w}(\cdot)=\left(w_{0}(\cdot), w_{1}(\cdot)\right) \in W^{1,2}\left[0, T ; \mathscr{H}^{*}\right]$ which implies that $A^{*} w_{1}(\cdot)=-\dot{w}_{0}(\cdot) \in L^{2}[0, T ; H]$ and hence $w_{1}(\cdot) \in L^{2}[0, T ; W]$. Furthermore, the output of (7.19) can be written in the simpler form $u(t)=-\mathscr{B} * \hat{w}(t)$ if $\mathscr{B}^{*}$ is understood as the extended operator on $H \times W$. Note that the SCS (7.19) is related to the second order Cauchy problem

$$
\left\{\begin{array}{l}
\frac{d}{d t}\left(\dot{w}(t)+C^{*} y(t)\right)=A^{*} w(t), \quad t \leqslant T, \\
u(t)=-B^{*} w(t), \quad w(0)=\psi^{1} \in V, \dot{w}(0)+C^{*} y(0)=-\psi^{0} \in H .
\end{array}\right.
$$

More precisely, if $w(\cdot) \in \mathscr{C}[0, T ; V] \cap W^{1,2}[0, T ; H]$ with $\dot{w}(\cdot)+C^{*} y(\cdot) \in$ $\mathscr{C}[0, T ; H] \cap \mathscr{C}^{1}\left[0, T ; V^{*}\right]$ satisfies $(7.20)$ then

$$
\hat{w}(\cdot)=\left(-\dot{w}(\cdot)-C^{*} y(\cdot), w(\cdot)\right) \in \mathscr{C}\left[0, T ; \mathscr{H}^{*}\right] \cap W^{1,2}\left[0, T ; \mathscr{W}^{*}\right]
$$

is the unique solution of (7.19) and vice versa. The output of (7.20) is only well defined in a strong sense if $w(\cdot) \in \mathscr{C}[0, T ; W]$ or, equivalently, $\dot{w}(\cdot)+C^{*} y(\cdot) \in$ $\mathscr{C}^{1}[0, T ; H]$. This will always be the case if the system is wellposed and $y(\cdot) \in$ $W^{1,2}[0, T ; \mathscr{Y}], w(0) \in W, \dot{w}(0) \in V$. Back in the special situation of (7.12) the dual system corresponds to the hyperbolic PDE

$$
\left\{\begin{array}{l}
\frac{\partial}{\partial t}\left(\frac{\partial w}{\partial t}+C^{*} y\right)(x, t)+\sum_{|\rho|,|\sigma| \leqslant m}(-1)^{|\sigma|} D^{\sigma}\left(a_{\rho \sigma} D^{\rho} w\right)(x, t)=0, \quad x \in \Omega, t \leqslant T, \\
u_{j}(x, t)=-B_{j}^{*} w(x, t), \quad x \in \partial \Omega, t \leqslant T, j=0, \ldots, m-1 .
\end{array}\right.
$$


Let us now consider the problem of minimizing the cost functional

$$
J(u)=\int_{0}^{T}\left[\|y(t)\|_{Y}^{2}+\|u(t)\|_{U}^{2}\right] d t
$$

subject to the SCS (7.15), respectively (7.14). In order to apply the results of $\S 5$ to this problem we assume that the SCS (7.15) is wellposed in the sense of Definition 2.4 and introduce the operator

$$
\Pi(t)=\mathscr{C}^{*}(T-t)\left[I+\mathscr{T}(T-t) \mathscr{T}^{*}(T-t)\right]^{-1} \mathscr{C}(T-t) \in \mathscr{L}\left(\mathscr{H}, \mathscr{H}^{*}\right)
$$

for $0 \leqslant t \leqslant T$. Also, for all $\varphi \in \mathscr{H}, \psi \in \mathscr{H}^{*}$ we denote by $z(\cdot ; T, \varphi, \psi) \in$ $\mathscr{C}[0, T ; H] \cap \mathscr{C}^{1}\left[0, T ; V^{*}\right] \cap W^{2,2}\left[0, T ; W^{*}\right], \quad u(\cdot ; T, \varphi, \psi) \in L^{2}[0, T ; \mathscr{U}]$, $y(\cdot ; T, \varphi, \psi) \in L^{2}[0, T ; \mathscr{Y}]$ and $w(\cdot ; T, \varphi, \psi) \in \mathscr{C}[0, T ; V] \cap W^{1,2}[0, T ; H]$ with $C z(\cdot ; T, \varphi, \psi) \in W^{1,2}[0, T ; \mathscr{Y}]$ and $\dot{w}(\cdot ; T, \varphi, \psi)+C^{*} y(\cdot ; T, \varphi, \psi) \in \mathscr{C}[0, T ; H] \cap$ $\mathscr{C}^{1}\left[0, T ; V^{*}\right]$ the unique solutions of the coupled equations (7.14), (7.20). Finally we introduce for $0 \leqslant t \leqslant T$ the space $\mathscr{W}(t)$ of all $\varphi \in Z \times H$ for which there exists a $y_{1} \in \mathscr{Y}$ with $C^{*} y_{1} \in V$ and

$$
\left\{\begin{array}{l}
\Gamma \varphi^{0}+\int_{0}^{T-t} u\left(s ; T-t,\left(\varphi^{1}, \Delta \varphi^{0}\right),\left(0,-C^{*} y_{1}\right)\right) d s=0 \\
C \varphi^{1}+\int_{0}^{T-t} y\left(s ; T-t,\left(\varphi^{1}, \Delta \varphi^{0}\right),\left(0,-C^{*} y_{1}\right)\right) d s=y_{1}
\end{array}\right.
$$

Then we obtain the following result as a consequence of Theorems 5.2, 5.3 and Corollary 5.8 .

COROLlary 7.8. Suppose that the SCS (7.15) is wellposed in the sense of Definition 2.4. Then the following statements hold.

(i) For every $\varphi \in \mathscr{H}$ there exists a unique optimal control $u(\cdot ; \varphi) \in L^{2}[0, T ; \mathscr{U}]$ which minimizes the cost functional (7.22) subject to (7.14). This optimal control is characterized by (7.20) with $\psi=0$. The optimal output of (7.14) is denoted by $y(t ; \varphi)$ and the corresponding solutions $z(t ; \varphi)$ and $w(t ; \varphi)$ of (7.14) and (7.20), respectively, satisfy

$$
\left(-\dot{w}(t ; \varphi)-C^{*} y(t ; \varphi), w(t ; \varphi)\right)=\Pi(t)(z(t ; \varphi), \dot{z}(t ; \varphi))
$$

for $0 \leqslant t \leqslant T$. The optimal cost is given by

$$
J(u(\cdot ; \varphi))=\langle\varphi, \Pi(0) \varphi\rangle_{\mathscr{H}, \mathscr{H} *} .
$$

(ii) $\varphi \in \mathscr{W}(0)$ if and only if $u(\cdot ; \varphi) \cap W^{1,2}[0, T ; \mathscr{U}], y(\cdot ; \varphi) \in W^{1,2}[0, T ; \mathscr{Y}]$, $z(\cdot ; \varphi) \in \mathscr{C}[0, T ; Z] \cap \mathscr{C}^{1}[0, T ; H] \cap \mathscr{C}^{2}\left[0, T ; V^{*}\right], \quad w(\cdot ; \varphi) \in \mathscr{C}[0, T ; W] \cap$ $\mathscr{C}^{1}[0, T ; V]$. If $\varphi \in \mathscr{W}(0)$ then $(z(t ; \varphi), \dot{z}(t ; \varphi)) \in \mathscr{W}(t)$ for $0 \leqslant t \leqslant T$ and

$$
u(t ; \varphi)=\Gamma z(t ; \varphi)=-B^{*} w(t ; \varphi)=-B^{*}[\Pi(t)(z(t ; \varphi), \dot{z}(t ; \varphi))]^{1} .
$$

The wellposedness for general systems of the form (7.12) seems to be an open problem. In fact, a spectral analysis of the case $m=2$ in a single space dimension indicates that the wellposedness in the space $\mathscr{H}=H \times V^{*}$ cannot be expected unless $u_{j}(t) \equiv 0$ for $j<m-1$. However, in the case $m=1$ with $L$ the Laplacian 
and Dirichlet boundary control, that is

$$
L \varphi=\sum_{1}^{n} \frac{\partial^{2} \varphi}{\partial x_{i}^{2}}, \quad B^{*} \psi=-D_{\nu} \psi,
$$

for $\varphi, \psi \in H^{2}(\Omega)$, the following nice result has been established by Lasiecka and Triggiani [26].

THEOREM 7.9 [26]. If $m=1$ and $L \in \mathscr{L}\left(H^{2}(\Omega), L^{2}(\Omega)\right), B^{*} \in \mathscr{L}\left(H^{2}(\Omega), L^{2}(\partial \Omega)\right)$ are given by (7.26) then the SCS (7.15) satisfies hypothesis $(\mathrm{S} 2)$ of $\$ 2.1$.

We conclude that the SCS (7.15) is in fact wellposed if (7.26) holds and $C \in \mathscr{L}\left(V^{*}, \mathscr{Y}\right)$ so that the above results can be applied in this situation. If $C \notin \mathscr{L}\left(V^{*}, \mathscr{Y}\right)$ then the output operator $\mathscr{C} \in \mathscr{L}(\mathscr{W}, \mathscr{Y})$ is unbounded with respect to the state space $\mathscr{H}=H \times V^{*}$. It seems to be a reasonable conjecture that the SCS (7.15) is still wellposed for a certain class of unbounded output operators.

We also mention the paper by Graham and Russell [18] which is concerned with regularity properties of the wave equation under Neumann boundary control.

Finally, we point out that analogous results on the linear quadratic control problem have been derived by Lasiecka and Triggiani [27] for system (7.12) with the cost functional

$$
J(u)=\int_{0}^{T}\left[\int_{\Omega} z(x, t)^{2} d x+\int_{\partial \Omega} u(x, t)^{2} d s(x)\right] d t
$$

under the assumptions of Theorem 7.9. This cost functional corresponds to the bounded output operator $\mathscr{C}=\left[\begin{array}{ll}I & 0\end{array}\right] \in \mathscr{L}(\mathscr{H}, H)$.

7.3. Two examples. In this section we briefly discuss two specific partial differential equations in a single space dimension. The essential feature of these systems is that the input and output operators are both strictly unbounded. In particular the wave equation (7.27) has been one of the main motivating examples for the development of the theory in $\$ 2$.

For $t>0$ consider the one dimensional second order hyperbolic PDE with Neumann boundary control and point observation in the velocity described by

$$
\left\{\begin{array}{l}
\frac{\partial^{2} z}{\partial t^{2}}(x, t)=a^{2} \frac{\partial^{2} z}{\partial x^{2}}(x, t), \quad 0<x<L, \\
z(0, t)=0, \quad \frac{\partial z}{\partial x}(L, t)=b u(t) \\
y(t)=c \frac{\partial z}{\partial t}(L, t)
\end{array}\right.
$$

where $u(t) \in R$ is the input and $y(t) \in R$ is the output. This system can be understood as a mathematical model for an undamped string with a fixed left end and the right end moving freely along a vertical line, the control acting through the angle at the right end. Solving equation (7.27) along its characteristics one can see that its input/output relationship with zero initial state is described by the difference equation

$$
y(t)+y(t-2 L / a)=a b c[u(t)-u(t-2 L / a)] .
$$


Using this fact in connection with results in Pritchard and Salamon [34] or Ho and Russell [19] one gets that system (7.27) is wellposed in the state space

$$
H=\left\{\left(\varphi^{0}, \varphi^{1}\right) \in H^{1}[0, L] \times L^{2}[0, L] \mid \varphi^{0}(0)=0\right\} .
$$

More precisely, this system can be described within the framework of $\S 2.2$ with

$$
\begin{aligned}
Z & =\left\{\left(\varphi^{0}, \varphi^{1}\right) \in H^{2}[0, L] \times H^{1}[0, L] \mid \varphi^{0}(0)=0, \varphi^{1}(0)=0\right\}, \\
U & =Y=R, \\
\Delta \varphi & =\left(\varphi^{1}, a^{2} \frac{\partial^{2} \varphi^{0}}{\partial x^{2}}\right), \quad \Gamma \varphi=b^{-1} \dot{\varphi}^{0}(L), \quad K \varphi=c \varphi^{1}(L) .
\end{aligned}
$$

and is wellposed in the sense of Definition 2.10. System (7.27) is of particular interest, since it can be stabilized throughh the static output feedback law

$$
u(t)=-f y(t), \quad f a b c>0,
$$

(Russell [39], Quinn and Russell [35]). Following Theorem 4.2, the wellposedness of the closed loop system (7.27), (7.29) requires the invertibility of the operator $I+f \mathscr{T}(t)$ for $t>0$. It follows from equation (7.28) that this operator is invertible if and only if $f a b c \neq-1$.

Our second example is the one dimensional fourth order hyperbolic PDE described by

$$
\left\{\begin{array}{l}
\frac{\partial^{2} z}{\partial t^{2}}(x, t)=-a^{4} \frac{\partial^{4} z}{\partial x^{4}}(x, t), \quad 0<x<L \\
z(0, t)=0, \quad \frac{\partial^{2} z}{\partial x^{2}}(L, t)=0 \\
\frac{\partial z}{\partial x}(0, t)=0, \quad \frac{\partial^{3} z}{\partial x^{3}}(L, t)=b u(t), \\
y(t)=c \frac{\partial z}{\partial t}(L, t)
\end{array}\right.
$$

where $u(t) \in R$ is the input and $y(t) \in R$ is the output. This system can be understood as a mathematical model for an undamped beam with a clampled left end and a free right end, with the control acting through an external force at the right end. System (7.30) can again be reformulated within the framework of $\$ 2.2$ with

$$
\begin{aligned}
& H=\left\{\left(\varphi^{0}, \varphi^{1}\right) \in H^{2}[0, L] \times L^{2}[0, L] \mid \varphi^{0}(0)=\dot{\varphi}^{0}(0)=0\right\}, \\
& Z=\left\{\left(\varphi^{0}, \varphi^{1}\right) \in H^{4}[0, L] \times H^{2}[0, L] \mid \varphi^{0}(0)=\dot{\varphi}^{0}(0)=\ddot{\varphi}^{0}(L)=0,\right. \\
&\left.\varphi^{1}(0)=\dot{\varphi}^{1}(0)=0\right\}, \\
& U=Y=R, \quad \Delta \varphi=\left(\varphi^{1},-a^{4} \frac{\partial^{4} \varphi}{\partial x^{4}}\right), \quad \Gamma \varphi=b^{-1} \frac{\partial^{3} \varphi^{0}}{\partial x^{3}}(L), \quad K \varphi=c \varphi^{1}(L) .
\end{aligned}
$$

The wellposedness problem for system (7.30) in the state space $H$ has apparently not been investigated in the open literature. However, a spectral analysis of the free system in connection with general wellposedness criteria in Pritchard and Salamon 
[34] or Ho and Russell [19] shows that the hypotheses (B2) and (B3) are satisfied. Hypothesis (B2) will no longer be satisfied if the control acts through the second derivative. The verification of hypothesis (B4) seems to involve some further technical difficulties and is left as a conjecture. The feedback stabilization problem for system (7.30) has been studied by Chen, Delfour, Krall and Payre [5]. They have shown via energy estimates that the system (7.30) can be exponentially stabilized through the static output feedback law

$$
u(t)=f y(t), \quad f b c>0 .
$$

\section{REFERENCES}

1. S. Agmon, A. Douglis and L. Nirenberg, Estimates near the boundary for solutions of elliptic partial differential equations satisfying general boundary conditions, Comm. Pure Appl. Math. 12 (1959), 623-727.

2. A. V. Balakrishnan, Boundary control of parabolic equations: $L-Q-R$ theory, Proc. Conf. Theory Nonlinear Equations, Akademie-Verlag, Berlin, 1978.

3. C. Bernier and A. Manitius, On semigroups in $R^{n} \times L^{p}$ corresponding to differential equations with delays, Canad. J. Math. 30 (1978), 897-914.

4. J. A. Burns, T. L. Herdman and H. W. Stech, Linear functional differential equations as semigroups in product spaces, SIAM J. Math. Anal. 14 (1983), 98-116.

5. G. Chen, M. C. Delfour, A. M. Krall and G. Payre, Modelling, stabilization and control of serially connected beams, Tech. Rep. Dept. of Math., Pennsylvania State Univ., University Park, Pa., 1984.

6. R. F. Curtain and A. T. Prichard, Infinite dimensional linear systems theory, LNCIS 8, Springer-Verlag, Berlin, 1978.

7. R. F. Curtain and D. Salamon, Finite dimensional compensators for infiite dimensional systems with unbounded control action, SIAM J. Control Optim. 24 (1986), 797-816.

8. G. DaPrato, Some results on linear stochastic evolution equations in Hilbert space by the semigroup method, Stochastic Anal. Appl. 1 (1983), 57-88.

9. R. Datko, Neutral autonomous functional equations with quadratic cost, SIAM J. Control 12 (1974), 70-82.

10. M. C. Delfour, The linear quadratic optimal control problem with delays in the state and control variables: a state space approach, SIAM J. Control Optim. 24 (1986), 835-883.

11. M. C. Delfour and J. Karrakchou, State space theory of linear time invariant systems with delays in state control and observation variables, Parts I, II, CRMA, Univ. de Montréal, CRMA-1223, 1984.

12. H. O. Fattorini, Boundary control systems, SIAM J. Control 6 (1968), 349-385.

13. F. Flandoli, Riccati equation arising in a boundary control problem with distributed parameters, SIAM J. Control Optim. 22 (1984), 76-86.

14. Dynamic programming approach to the optimal control of systems governed by nonwellposed Cauchy problems in Hilbert spaces, Second Internat. Conf. on Control Theory for Distributed Parameter Systems, (F. Kappel, K. Kunisch and W. Schappacher, eds.), July, 1984.

15. G. B. Folland, Introduction to partial differential equations, Princeton Univ. Press, Princeton, N. J., 1976.

16. A. Friedman, Partial differential equations, Holt, Rinehart and Winston, 1969.

17. J. S. Gibson, The Riccati integral equations for optimal control problems in Hilbert space, SIAM J. Control Optim. 17 (1979), 537-565.

18. K. D. Graham and D. L. Russell, Boundary value control if the wave equation in a spherical region, SIAM J. Control Optim. 13 (1975), 174-196.

19. L. F. Ho and D. L. Russell, Admissable input elements for systems in Hilbert space and a Carleson measure criterion, SIAM J. Control Optim. 21 (1983), 614-640.

20. A. Ichikawa, Quadratic control of evolution equations with delays in control, SIAM J. Control Optim. 20 (1982), 645-668.

21. K. Ito and T. J. Tarn, A linear quadratic control problem for neutral systems, Nonlinear Anal. (to appear).

22. J. Karrakchou, Analyse et commande systèmes differentiels fonctionels de type héréditaire, Thèse de doctorat, Univ. de Montréal, CRMA-1205, 1984. 
23. I. Lasiecka, Unified theory for abstract parabolic boundary problems: a semigroup approach, Appl. Math. Optim. 6 (1980), 287-333.

24. I. Lasiecka and R. Triggiani, A cosine operators approach to modelling $L^{2}\left[0, T ; L^{2}(\Gamma)\right]$-boundary input hyperbolic equations, Appl. Math. Optim. 7 (1981), 35-93.

25. Dirichlet boundary control problem for parabolic equations with quadratic cost: analyticity and Riccati's feedback synthesis, SIAM J. Control Optim. 21 (1983), 41-67.

26. Regularity of hyperbolic equations under $L^{2}\left[0, T ; L^{2}(\Gamma)\right]$ - Dirichlet boundary terms, SIAM J. Control Optim. 24 (1986), 884-925.

27.,$A n L^{2}$-theory for the quadratic optimal cost problem of hyperbolic equations with control in the Dirichlet boundary conditions, Control Theory for Distributed Parameter Systems and Applications (F. Kappel, K. Kunisch and W. Schappacher, eds.), LNCIS 54, Springer-Verlag, Berlin, 1983, pp. 138-152.

28. J. L. Lions, Optimal control of systems governed by partial differential equations, Springer-Verlag, New York, 1971.

29. J. L. Lions and E. Magenes, Nonhomogeneous boundary value problems and applications, vols. I, II, Springer-Verlag, New York, 1972.

30. D. Lukes and D. L. Russell, The quadratic criterion for distributed systems, SIAM J. Control Optim. 7 (1969), 101-121.

31. R. K. Miller, Linear Volterra integro-differential equations as semigroups, Funkcial. Ekvac. 17 (1974), 39-55.

32. A. Pazy, Semigroups of linear operators and applications to partial differential equations, SpringerVerlag, New York, 1984.

33. R. S. Phillips, A note on the abstract Cauchy problem, Proc. Nat. Acad. Sci. U.S.A. 40 (1954), 244-248.

34. A. J. Prichard and D. Salamon, The linear quadratic control problem for infinite dimensional systems, Part I: A semigroup theoretic approach for systems with unbounded input and output operators, SIAM J. Control Optim. (to appear); Part II: Retarded systems with delays in control and observation, IMA J. Math. Control Inf. 2 (1985), 335-362

35. J. P. Quinn and D. L. Russell, Asymptotic stability and energy decay rates for solutions of hyperbolic equations with boundary damping, Proc. Roy. Soc. Edinburgh Ser. A 77 (1977), 97-127.

36. D. L. Russell, On boundary value controllability of linear symmetric hyperbolic systems, Mathematical Theory of Control, Academic Press, New York, 1967, pp. 312-321.

37. __ Quadratic performance criteria in boundary control of linear symmetric hyperbolic systems, SIAM J. Control Optim. 11 (1973), 475-509.

38. _ A Anified boundary controllability theory for hyperbolic and parabolic partial differential equations, Stud. in Appl. Math. 52 (1973), 189-211.

39. Controllability and stabilizability theory for linear partial differential equations: recent progress and open question, SIAM Rev. 20 (1978), 639-739.

40. D. Salamon, Control and observation of neutral systems, Pitman, London, 1984.

41. A duality principle for neutral functional differential equations, EQUADIFF 82 (K. Schmitt and H. W. Knobloch, eds.), Lecture Notes in Math., vol. 1017, Springer, New York, 1983, pp. 543-552.

42. M. Sorine, Une resultat d'existence et unicité pour l'équation de Riccati stationaire, Rapport INRIA no. $55,1981$.

43. __ Sur le semigroupe non linéaire associe à l'équation de Riccati, CRMA, Univ. de Montréal, CRMA-1055, 1981.

44. R. B. Vinter and R. H. Kwong, The infinite time quadratic control problem for linear systems with state and control delays: an evolution equation approach, SIAM J. Control Optim. 19 (1981), 139-153.

45. D. Washburn, $A$ bound on the boundary input map for parabolic equations with applications to time optimal control, SIAM J. Control Optim. 17 (1979), 652-671.

Mathematics Research Center, University of Wisconsin, Madison, Wisconsin 53706

Current address: Mathematics Institute, University of Warwick, Coventry CV4 7AL, England 